\title{
Sensitivity analysis of DP4+ with the probability distribution terms: Development of a universal and customizable method
}

\author{
María Marta Zanardi $i^{a}$ and Ariel M. Sarotti ${ }^{b}$ * \\ ${ }^{a}$ Instituto de Ingeniería Ambiental, Química y Biotecnología Aplicada (INGEBIO), Facultad de Química e Ingeniería del \\ Rosario, Pontificia Universidad Católica Argentina, S2002QEO Rosario, Argentina \\ ${ }^{\mathrm{b}}$ Instituto de Química Rosario (CONICET), Facultad de Ciencias Bioquímicas y Farmacéuticas, Universidad Nacional de \\ Rosario, Suipacha 531, 2000 Rosario, Argentina.
}

sarotti@iquir-conicet.gov.ar

\section{List of contents}

1- Workflow of DP4+ and general recommendations, instructions for using the Excel file to compute the DP4+ probabilities (with tutorial). Pages S2-S10.

2- Computational methods. Pages S11.

3- Compounds used in the test set. Pages S12-S16.

4- $[\mu, \sigma, v]$ parameters estimated at different levels of theory. Pages S17-S18.

5- Changes in the DP4+ values calculated for the test set when correlating the GIAO NMR chemical shifts computed at the $\mathrm{PCM} / \mathrm{mPW} 1 \mathrm{PW} 91 / 6-31+\mathrm{G}^{* *} / / \mathrm{B} 3 \mathrm{LYP} / 6-31 \mathrm{G}^{*}$ level of theory, with the $[\mu, \sigma, v]$ parameters corresponding to different levels of theory. Pages S19-S21.

6- Ranges employed for the determination of the dependence of DP4+ with $[\mu, \sigma, v]$ (Figure 3b), and for the random generation of $[\mu, \sigma, v]$. Page S22.

7- Dependence of DP4+ with each of its $16[\mu, \sigma, v]$ parameters determined by correlating the GIAO NMR chemical shifts computed at the PCM/mPW1PW91/6-31+G**//B3LYP/6-31G* level of theory (M3P), with $15[\mu, \sigma, v] 0$ parameters estimated at the M3P level and allowing the remaining one to oscillate between 6 levels. Pages S23-S29.

8- Changes in the DP4+ values calculated for the test set (Figure 2) when correlating the GIAO NMR chemical shifts computed at the PCM/mPW1PW91/6-31+G**//B3LYP/6-31G* level of theory (M3P), with randomly generated $[\mu, \sigma, v]$ parameters. Pages S30-S32.

9- $[\mu, \sigma]$ values estimated using the reduced set of 8 molecules (Figure 5), and the full set of 77 molecules at 24 levels of theory. Page S33.

10- DP4+ values calculated for the test set (Figure 2) when correlating the GIAO NMR chemical shifts computed at the $\mathrm{PCM} / \mathrm{mPW} 1 \mathrm{PW} 91 / 6-31+\mathrm{G}^{* *} / / \mathrm{B} 3 \mathrm{LYP} / 6-31 \mathrm{G}^{*}$ level of theory (M3P) with the $[\mu, \sigma, v]_{0}$ estimated from the full set of 77 molecules at the same level of theory; b) using the $[\mu, \sigma]$ terms estimated from the reduced set of 8 molecules (Figure 5) and the $[v]$ terms estimated from the full set of 77 molecules at the same level of theory; and c) using the $[\mu, \sigma]$ terms estimated from the reduced set of 8 molecules (Figure 5) at the same level of theory and the averaged [v] terms of the 24 different levels of theory originally evaluated. Page S34.

11- Averaged degrees of freedom for the 24 levels of theory evaluated in the original method. Page S34.

12- Cartesian coordinates of compounds 49-56 optimized at the B3LYP/6-31G* level. Pages S35-S39. 


\section{Workflow of DP4+ and general recommendations}

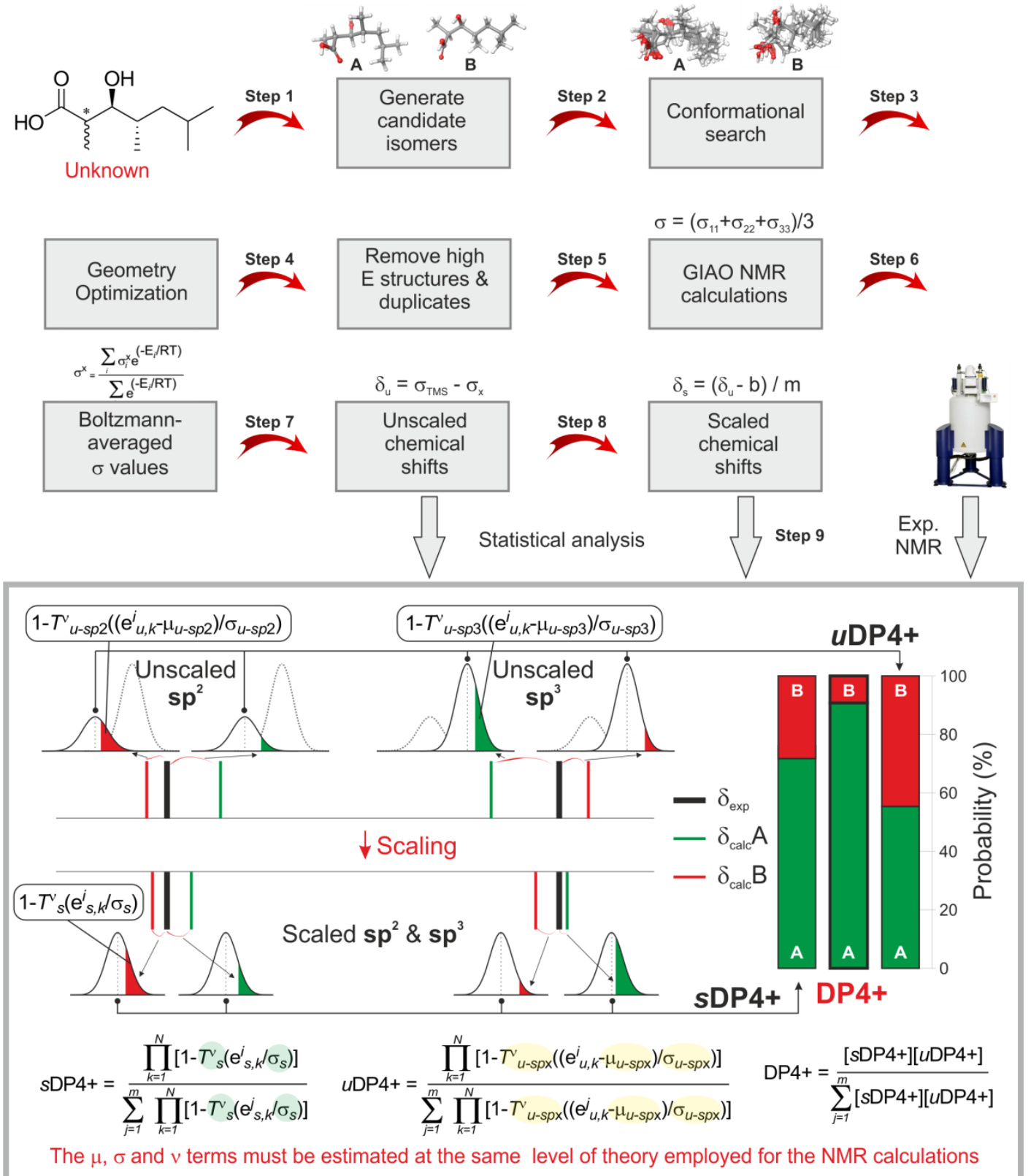

Step 1: Despite the new DP4+ spreadsheet can handle up to 128 isomers, keeping the number of candidates to a minimum has several advantages, as reduces both the overall computational cost and the probability that the calculated data for an incorrect isomer ends up having better fit with the experimental values than that for the correct candidate.

Step 2: The conformational search should provide a good description of the conformational landscape of the system under study. Improper computational work might lead to potentially negative consequences in the overall results. Systematic sampling is always recommended, but impractical in flexible molecules. In those cases, stochastic searches using a reasonably large number of steps should be carried out. To avoid missing potentially relevant conformations, all conformations within a safe energy window from the corresponding global minimum should be kept. For broad applications, a $5 \mathrm{kcal} / \mathrm{mol}$ cutoff value using MMFF, OPLS3 or equivalent force fields is recommended.

Step 3: In the original DP4+ method, the geometry optimizations should be done at the B3LYP/6-31G* level. In the new DP4+ method, there is no restriction regarding the level of theory (as long as the $[\mu, \sigma, v]$ parameters are properly estimated at this level). However, we recommend optimizing the geometries at DFT level using at least the 6-31G* basis set (or equivalent). For broad 
applications, popular functionals such as B3LYP, M06-2X, and WB97XD (among others) could be used. It is recommended running frequency calculations to determine the nature of the stationary point found.

Step 4: During the geometry optimization step, it is common that two (or more) conformations converge to the same structure. Hence, it is important to remove such duplicate structures. In addition, to reduce the computational cost in the next step, high energy conformations can be removed as well. For broad applications, a $5 \mathrm{kcal} / \mathrm{mol}$ cutoff value is recommended (using the relative energies computed at the geometry optimization level).

Step 5: In the new DP4+ method the NMR calculation step can be carried out at any desired level of theory (as long as the [ $\mu, \sigma, v]$ parameters are properly estimated at this level). For broad applications, we recommend using the GIAO method to tackle the gauge origin problem, with popular DFT functionals such as B3LYP, mPW1PW91, WB97XD, and PBE0 (among others) and at least the 6$31+G^{* *}$ basis set, including the solvent effects with PCM or related solvation model.

Steps 6: The Boltzmann-averaged shielding values should be computed as described in the computational details.

Step 7: The unscaled chemical shifts are computed as described in the computational details. If a new level of theory is to be used, it is important to compute the ${ }^{1} \mathrm{H}$ and ${ }^{13} \mathrm{C} \sigma$ values of the reference standard (TMS) at the exact same levels of theory used for the target compounds (including geometry optimization and NMR calculation steps). Using improper $\sigma$ values for TMS would affect the unscaled chemical shifts values, hence impacting in the $u \mathrm{DP} 4+$ term. If the molecule contains carbon attached to $\mathrm{Br}$ (or related heavy atoms) the errors are larger than the average due to the heavy atom effect. It would be recommended to exclude those carbons of the DP4+ calculations.

Step 8: The scaled chemical shifts are computed as described in the computational details. In order to prevent inadvertent mistakes, it is highly recommended using assigned experimental NMR data in order to properly match the experimental and calculated values for each nucleus. Alarmingly high errors (especially in the highest ranked compound) should trigger an in-depth review of the experimental and calculated data in search of mistakes. If those large errors persist, it should be considered the possibility that the correct structure was not included in the candidate set.

Step 9: It is essential to respect the rules for the Excel spreadsheet data entry. Improper selections in the drop down lists, not indicating the sp-sp2 nuclei, filling the Region B with improper $[\mu, \sigma, v]$, among other mistakes, would impact in the overall DP4+ results.

Note: if the $[\mu, \sigma, v]$ parameters are unknown for the level of theory chosen for the geometry optimization and/or the NMR calculation steps, they should be estimated at the same level either using the reduced set of molecules shown in Figure 5 (for preliminary calculations) or a larger set of known molecules. See the further instructions and tutorials for more information. 


\section{Instructions for using the new Excel file to compute the DP4+ probability}

- In order to simplify the new DP4+ calculations, an Excel file is provided from the authors which can be downloaded from:

http://www.sarotti-NMR.weebly.com

http://www.iquir-conicet.gov.ar/Sarotti-NMR

- The file contains 7 sheets (Figure S1).

- Main: contains the selection of the level of theory, and enabled cells to introduce the experimental and calculated NMR data.

- Unscaled: shows the unscaled chemical shifts, computed according to $\delta_{\text {calc }}=\sigma_{\mathrm{TMs}}-\sigma_{\mathrm{x}}$

- Scaled: shows the scaled chemical shifts, computed according to $\delta_{\mathrm{s}}=\left(\delta_{\text {calc }}-b\right) / m$

- Unscaled Errors: shows the unscaled errors, computed according to $e_{u}=\delta_{\text {calc }}-\delta_{\text {exp }}$

- Scaled Errors: shows the scaled errors, computed according to $e_{s}=\delta_{s}-\delta_{\exp }$

- Detailed Results: shows the scaled, unscaled, and full DP4+ results (sDP4+, uDP4+, and DP4+, respectively).

- Parameters Estimation: contains the experimental data of the 8 selected molecules shown in Figure 5, and enabled cells to introduce the calculated NMR shifts required to estimate the $[\mu, \sigma]$ terms.

\section{The "Main" sheet}

- The "Main" sheet contains the enabled cells to introduce the experimental and calculated NMR data, and provides the results of the DP4+ calculations. It is divided in 4 different regions: $A, B, C$, and $D$.

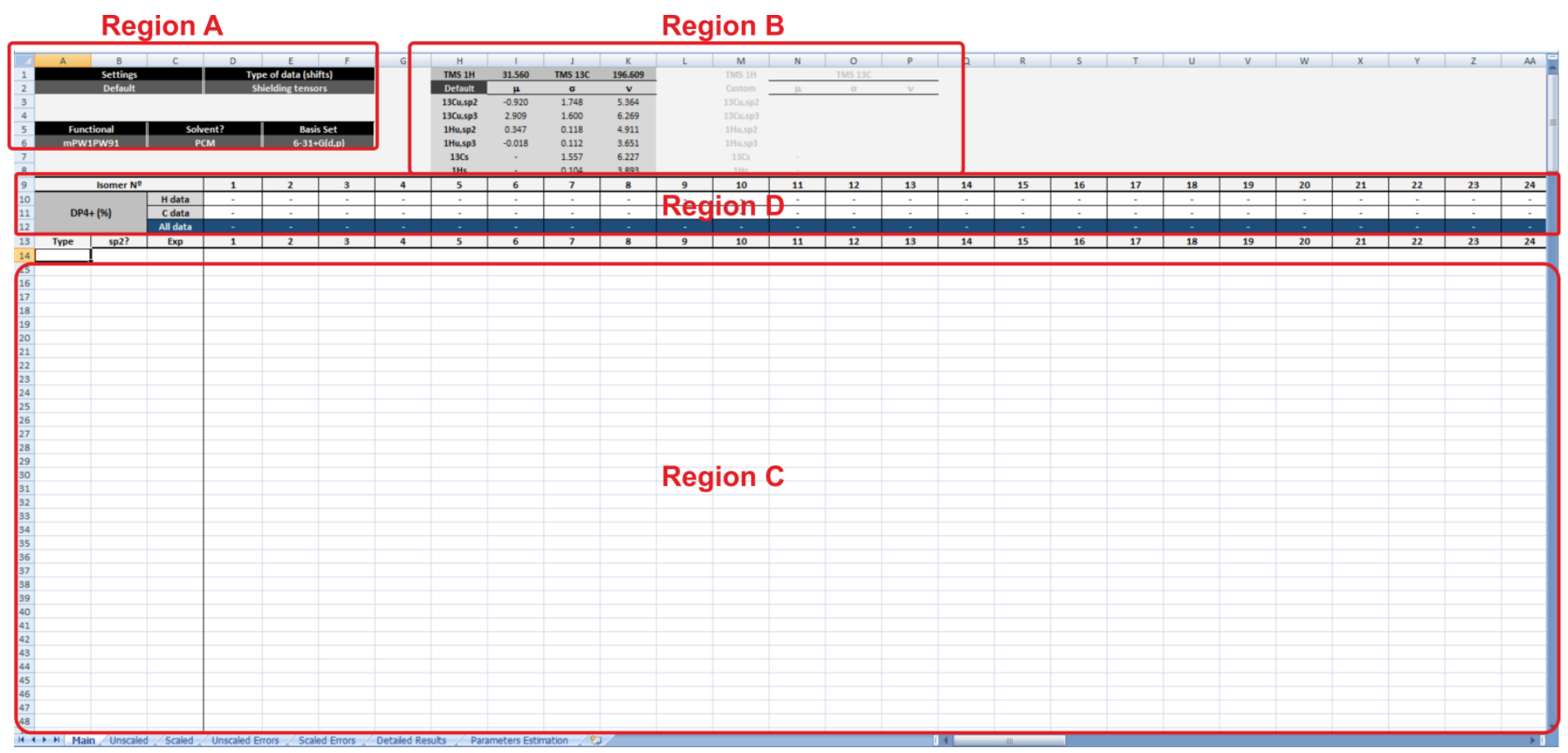

Figure S1

\section{Region A: user selection (drop down lists):}

- Settings: settings to be employed to compute the DP4+ probability (Default or Custom). The choice will be reflected in Region B.

- "Default" option allows to use the $[\mu, \sigma, v]$ terms calculated for a large set of 77 molecules at the 24 levels of theory evaluated in the original publication (JOC 2015, 80, 12526). If this option is selected, a new list is displayed with the following options:

- Functional: functional used to perform the GIAO NMR calculations (B3LYP or mPW1PW91).

- Solvent?: inclusion of solvent effect in the GIAO NMR calculations (PCM or Gas Phase). 
- Basis Set: basis set used in the GIAO NMR calculations (6-31G*, 6-31G**, 6-31+G**, 6-311G*, 6-311G** or 6$\left.311+G^{* *}\right)$.

After the selection is done, the corresponding $[\mu, \sigma, v]$ values and the isotropic shielding values for the reference standard (Tetramethylsilane, TMS) will be displayed in Region B.

- "Custom" option allows to set the values of the $[\mu, \sigma, v]$ terms, which could be estimated in different ways. For example, using the full set of 77 molecules evaluated in the original publication at different levels of theory, using a large set of different molecules, or using the reduced set of 8 molecules shown in Figure 5 for preliminary calculations. If this option is selected, the 16 values of the $[\mu, \sigma, v]$ set and the isotropic shielding values for the reference standard (TMS) should be provided in the enabled cells of Region $\mathrm{B}$.

\section{Region B: settings to compute the DP4+ probability}

Contains the $[\mu, \sigma, v]$ parameters required to perform DP4+ calculations. These values depend upon the level of theory employed during the calculations, and can be introduced in two different ways:

- If "Default" option is selected in Region A, the Default settings of the selected level of theory will be shown in Region B.

- If "Custom" option is selected in Region A, the Custom settings will be highlighted in Region B. These settings can be changed freely depending on the user preferences (for example, those obtained by using other levels of theory and/or a different set of test molecules). Warning: to obtain meaningful results, the modified parameters must be estimated at the desired level of theory using a large set of known compounds, or the recommended set of 8 molecules for preliminary calculations.
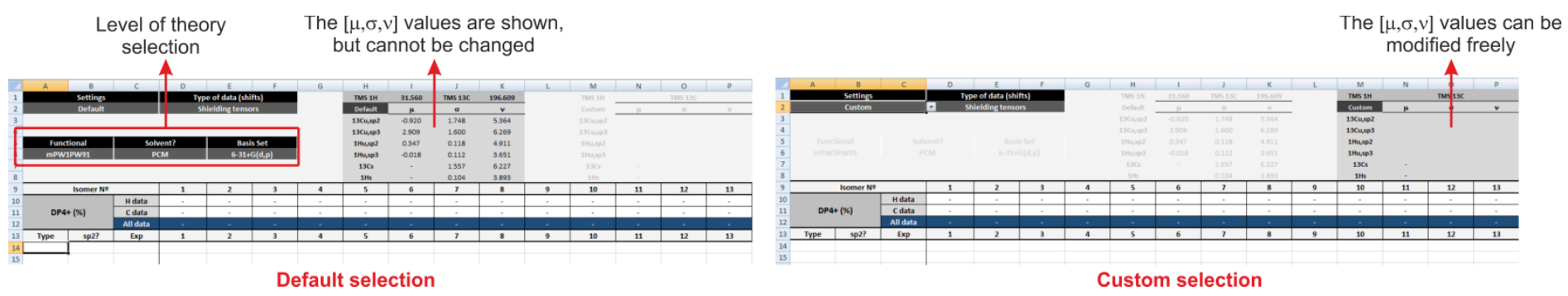

\section{Region C: enabled cells to enter the data:}

- Nuclei: enter the letter "C" (without quotes) for ${ }^{13} \mathrm{C}$ data or the letter "H" (without quotes) for ${ }^{1} \mathrm{H}$ data.

- sp2?: enter the letter " $\mathrm{x}$ " (without quotes) if the carbon is $\mathrm{sp}$-sp ${ }^{2}$ hybridized (or if the proton is attached to an $\mathrm{sp}$-sp ${ }^{2}$ hybridized carbon).

- Experimental: enter the experimental ${ }^{13} \mathrm{C}$ and/or ${ }^{1} \mathrm{H}$ chemical shifts.

- Isomer 1, Isomer 2, etc: enter the computed data for the candidate structures (either as isotropic shielding values, unscaled chemical shifts or scaled chemical shifts).

Region D: displays the DP4+ probabilities once the data are entered in Regions A, B and C. 
Tutorial 1: DP4+ calculations using the "Default" option.

Calculated NMR data: compounds T1-T8 at the PCM/mPW1PW91/6-31+G**//B3LYP/6-31G* level.

Experimental NMR data from compound T1 (correct isomer). Synthesis 2019, 51, 1545.
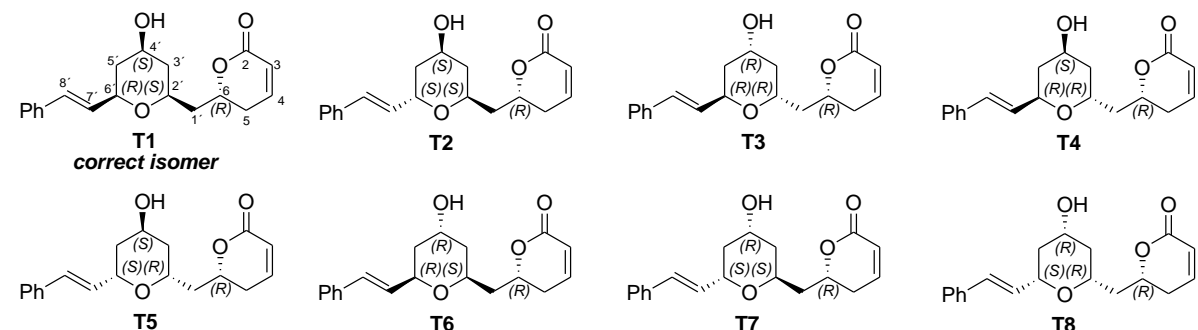

Step 1: perform a full conformational search using a suitable package (Hyperchem, Spartan, MacroModel, etc). It is recommended to keep all conformations within $5 \mathrm{kcal} / \mathrm{mol}(\sim 21 \mathrm{~kJ} / \mathrm{mol})$ from the global minimum.

Step 2: optimize all conformations found at the B3LYP/6-31G* level (gas phase). Remove duplicates if necessary.

Step 3: perform the NMR GIAO calculations at the selected level of theory (in this case, at the PCM/mPW1PW91/6-31+G**) for all representative conformations. Compute the Boltzmann-averaged shielding values for each compound.

Step 4: open the Excel file, and select the "Default" and "Shielding tensors" options in Region A, and select the level of theory that was used to perform the NMR calculations. In this case, Functional > mPW1PW91; Solvent > PCM; Basis set > 6-31+G**.

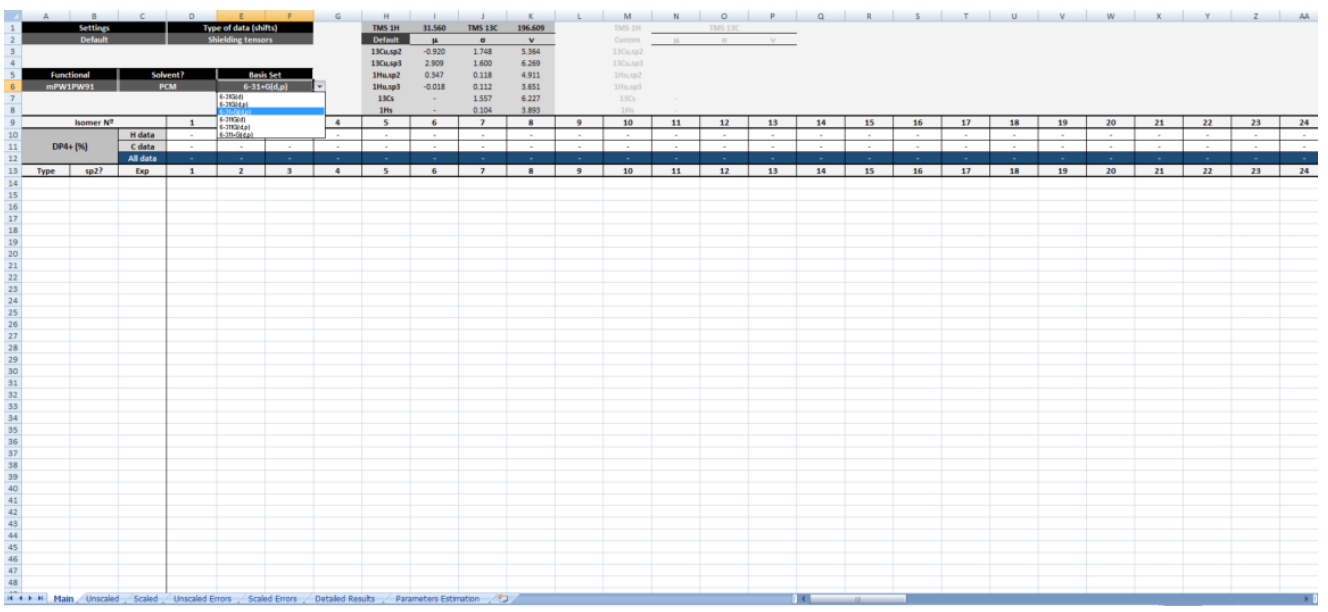

Step 5: Introduce the experimental data. Important: mark the sp ${ }^{2}$ nuclei with " $x$ ". Otherwise, improper distributions will be used for those nuclei, with potentially negative effects in the DP4+ values.

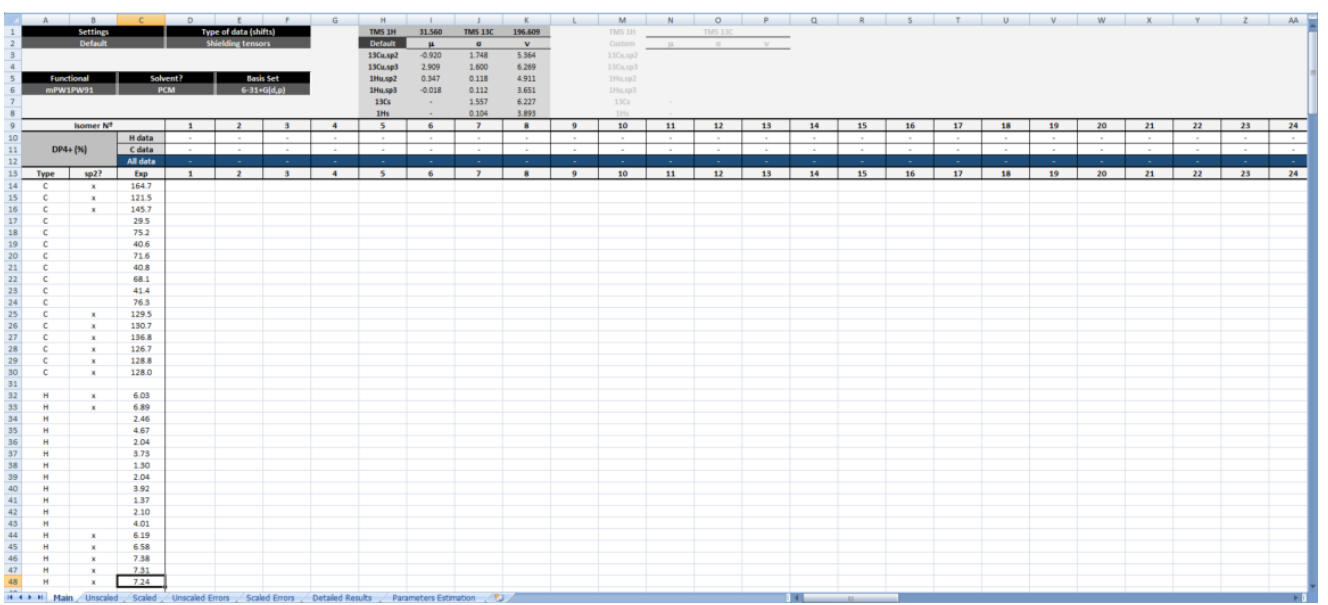


Step 6: Introduce the calculated averaged isotropic shielding values for each possible candidate structure ( 8 in this case). The data can be inserted manually or copy-pasted. The DP4+ values are automatically calculated and displayed for each isomer above the number of isomer. Important: the NMR data must be assigned (know which shift corresponds to which nuclei). Using unassigned or misassigned NMR data might lead to erroneous results.

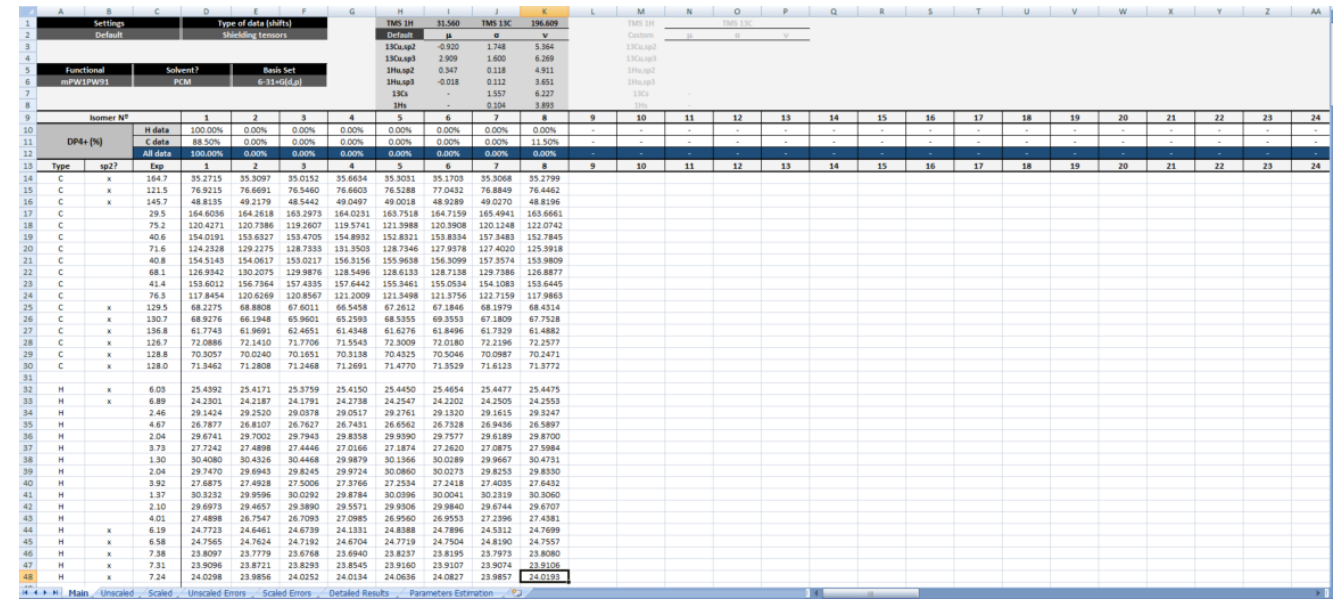

Warning: do not cut-paste information in this Excel file since it modifies the cell references for the hidden equations).

Once the Boltzmann-averaged isotropic shielding values are introduced, the calculated chemical shifts (both unscaled and scaled) are shown in the Unscaled and Scaled sheets, respectively. The corresponding errors can are shown as well in the Unscaled and Scaled errors sheets, respectively.
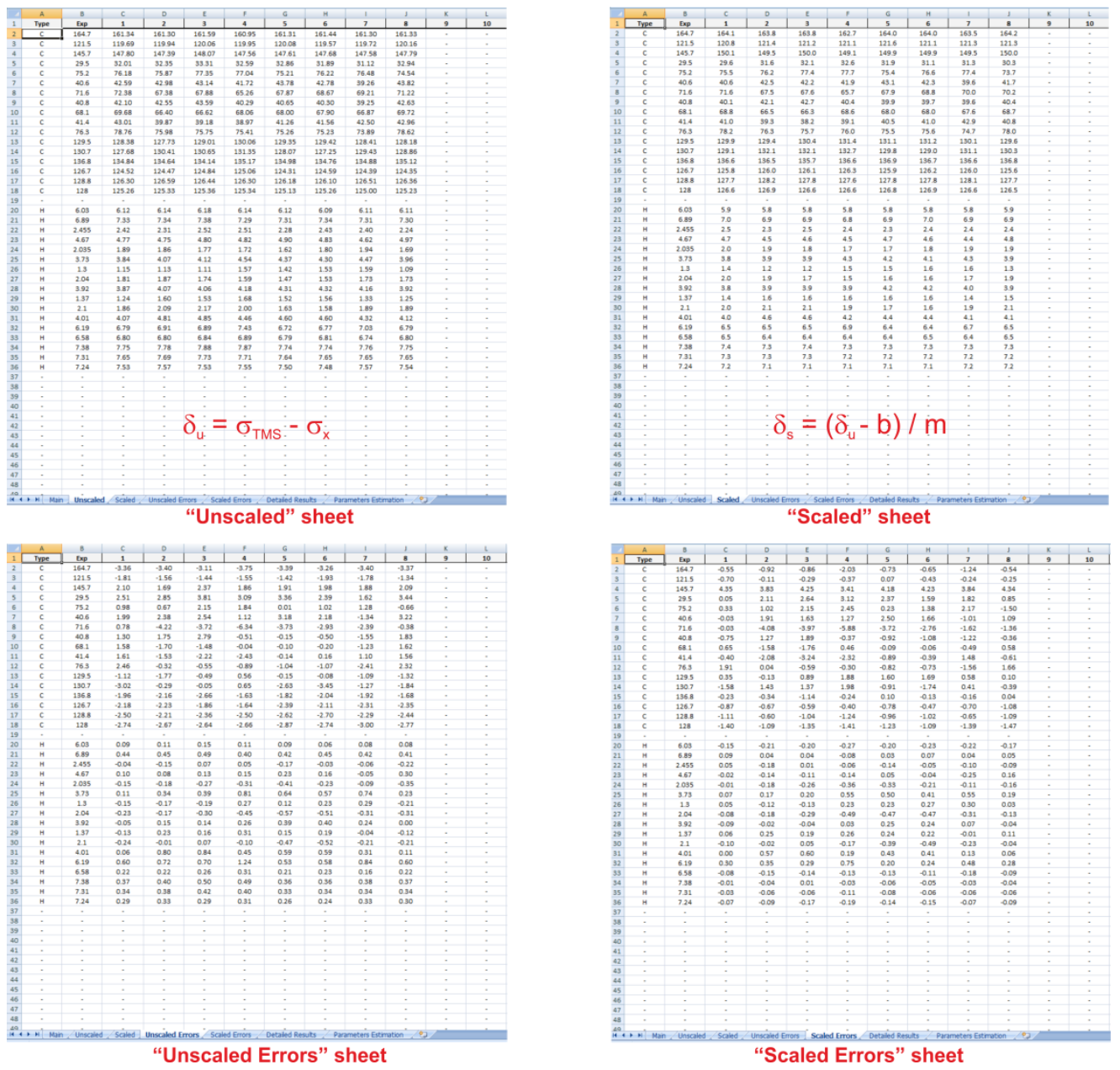
The probabilities computed using unscaled ( $u \mathrm{DP} 4+)$ and scaled (sDP4+), as well as using carbon and/or proton data, is shown in the sheet "Detailed Results"

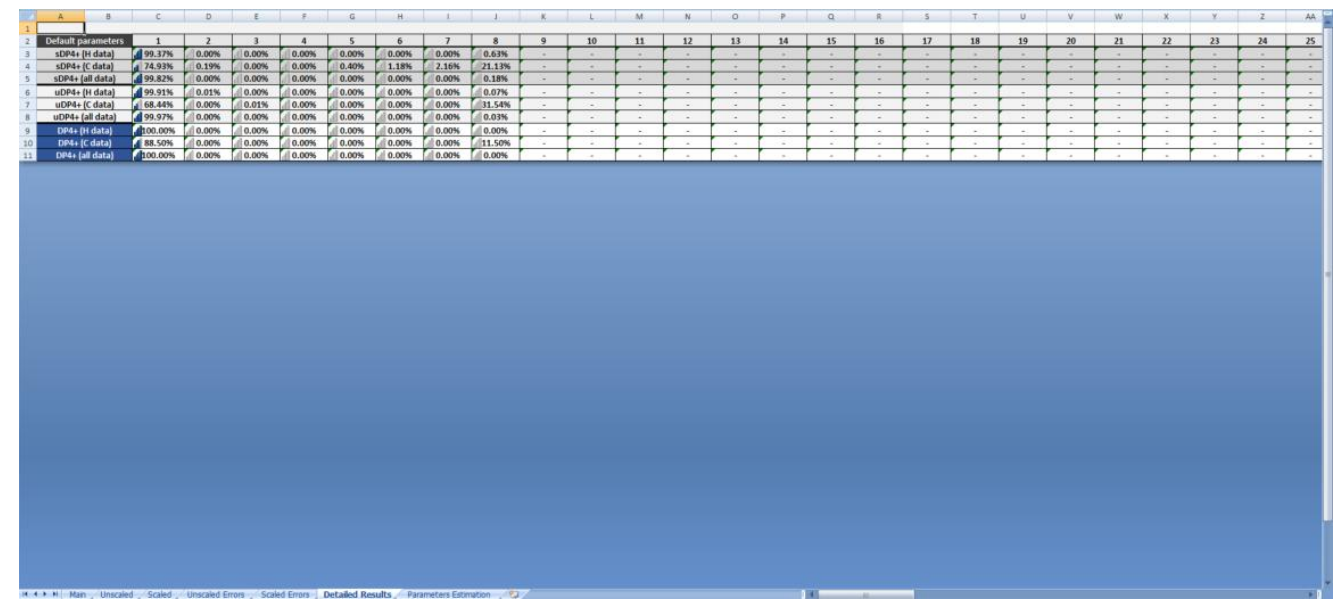


Tutorial 2: DP4+ calculations using the "Custom" option.

Calculated NMR data: compounds Ta1-Ta2 at the PCM/

Experimental NMR data from compound Ta1 (correct isomer). JOC 2015, 80, 12526

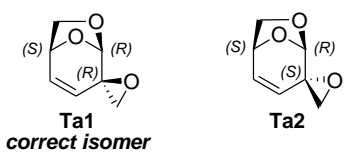

Step 1: The computational work should be done as described in the Tutorial 1 , using the M06-2X/6-31+G** level for the geometry optimization step, and the PCM/ $\mathrm{BB97XD/6-311+G**}$ for the GIAO NMR calculation step.

Step 2: open the Excel file, and select the "Custom" and "Shielding tensors" options in Region A. This will activate the edition of the $[\mu, \sigma, v]$ values in Region B.

Step 3: compute the isotropic shielding values for the reference standard (TMS) at the same level of theory employed for the test compounds (PCM/ $\left.\mathrm{BB} 97 \mathrm{XD} / 6-311+\mathrm{G}^{* *} / / \mathrm{M} 06-2 \mathrm{X} / 6-31+\mathrm{G}^{* *}\right)$, and introduce those values in the upper region of Region $\mathrm{B}$.
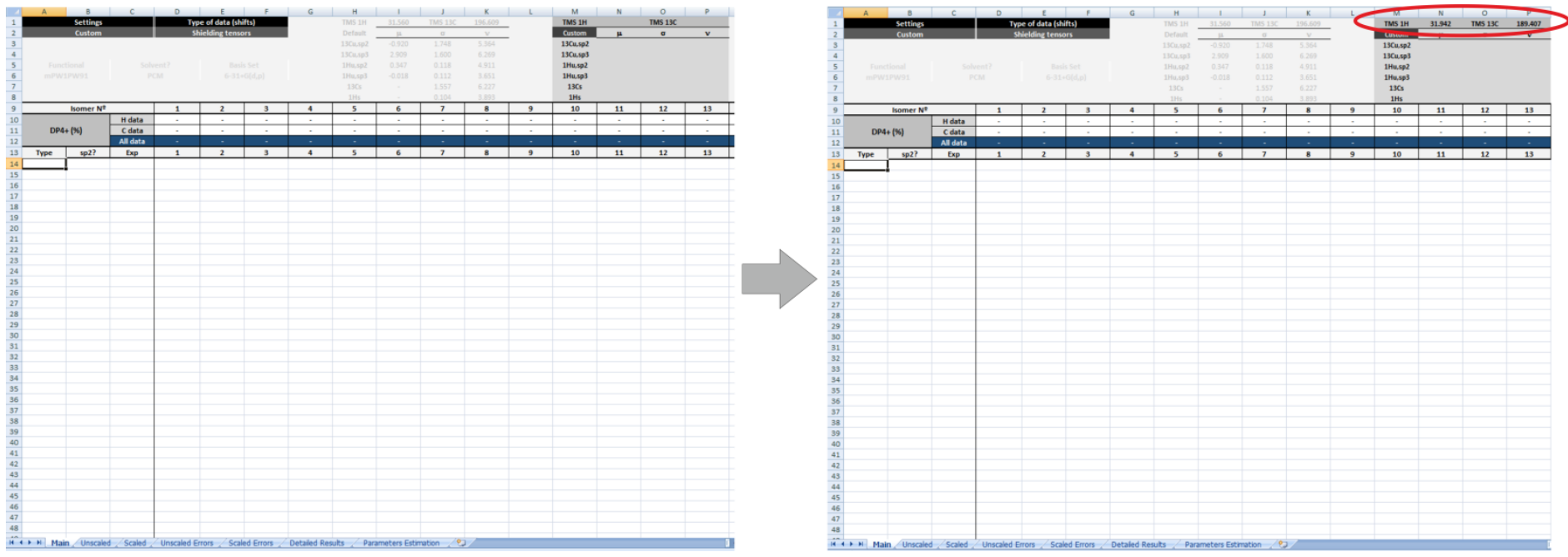

Step 4: if the $[\mu, \sigma, v]$ parameters have been previously estimated using a large set of molecules at the selected level of theory, fill the data in the lower part of Region B. If not, for preliminary calculations the values can be estimated with the 8 molecules shown in Figure 5. The NMR chemical shifts of compounds 49-56 must be computed at the PCM/ $1 \mathrm{~B} 97 \mathrm{XD} / 6-311+\mathrm{G} * * / / \mathrm{M} 06-2 \mathrm{X} / 6-31+\mathrm{G} * *$ level of theory, and the data must be filled in the "Parameters Estimation" sheet. Once all the $\sigma_{x}$ values are introduced (along with the $\sigma_{0}$ values of TMS), the unscaled and scaled chemical shifts will be automatically computed, and the [ $\left.\mu, \sigma\right]$ values will be shown. Important: the NMR data must be assigned (know which shift corresponds to which nuclei). It is essential to respect the assignment indicated in the Sheet. Using other assignments will lead to erroneous results.
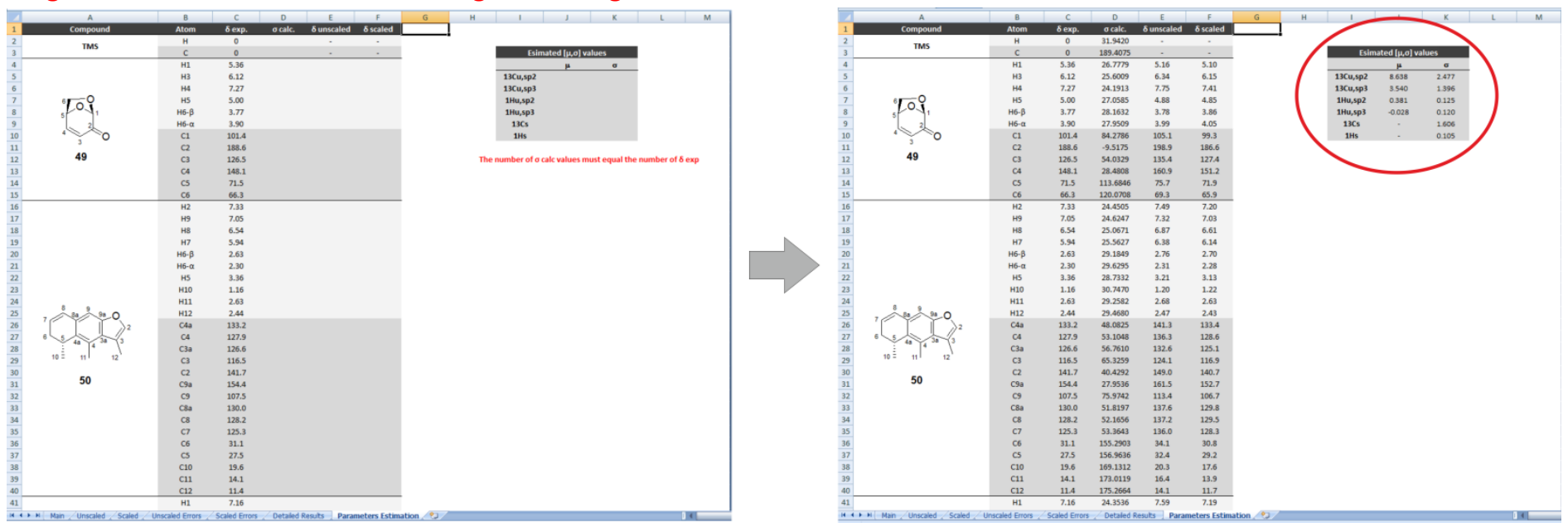
Step 5: complete the Region B with these $[\mu, \sigma]$ values estimated in the previous step. Regarding the degrees of freedom (v), for preliminary estimations can be used the averaged values from the 24 levels of theory previously evaluated (see Table S14). Those values are: $v_{13 C u, s p 2}=8 ; v_{13 C u, s p 3}=10 ; v_{1 H u, s p 2}=8 ; v_{1 H u, s p 3}=4 ; v_{13 C s}=7$; and $v_{1 H s}=4$.

Step 6: complete the Region C with the experimental and calculated NMR data. The DP4+ values are shown in Region C.
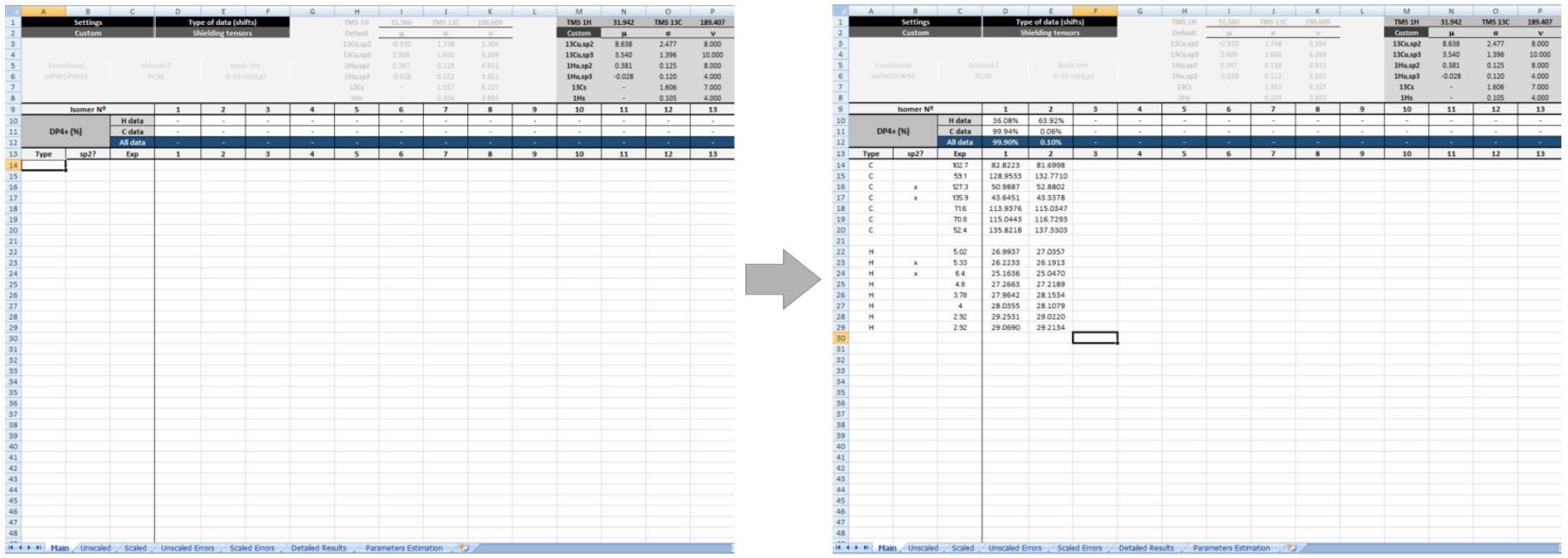
Computational Methods. All the quantum mechanical calculations were performed using Gaussian 09. ${ }^{1}$ Systematic conformational searches were done for each compound in the gas phase using the MMFF force field implemented in Macromodel. ${ }^{2}$ The MMFF geometries were optimized at the indicated level of theory. Frequency calculations were done for all optimized geometries at the same level to determine the nature of the stationary point found. After removing duplicates, the optimized geometries were next subjected to GIAO NMR calculations at the indicated level of theory. In all cases, the unscaled chemical shifts $\left(\delta_{\mathrm{u}}\right)$ were computed using TMS as reference standard according to $\delta_{\mathrm{u}}=\sigma_{0}-$ $\sigma_{\mathrm{x}}$, where $\sigma_{\mathrm{x}}$ is the Boltzmann averaged shielding tensor (over all significantly populated conformations) and $\sigma_{0}$ is the shielding tensor of TMS computed at the same level of theory employed for $\sigma_{\mathrm{x}}$. The Boltzmann averaging was done according to eq 1 :

$$
\sigma^{\mathrm{x}}=\frac{\sum_{i} \sigma_{i}^{\mathrm{x}} \mathrm{e}^{\left(-\mathrm{E}_{i} / \mathrm{RT}\right)}}{\sum_{i} e^{\left(-\mathrm{E}_{i} / \mathrm{RT}\right)}}
$$

where $\sigma_{i}^{x}$ is the shielding constant for nucleus $x$ in conformer $i, R$ is the molar gas constant $(8.3145 \mathrm{~J}$ $\left.\mathrm{K}^{-1} \mathrm{~mol}^{-1}\right), T$ is the temperature $(298 \mathrm{~K})$, and $E_{i}$ is the SCF energy of conformer $i$ (relative to the lowest energy conformer), obtained at the corresponding level of theory. The scaled chemical shifts $\left(\delta_{s}\right)$ were computed as $\delta_{s}=\left(\delta_{u}-b\right) / m$, where $m$ and $b$ are the slope and intercept, respectively, resulting from a linear regression calculation on a plot of $\delta_{u}$ against $\delta_{\text {exp }}$.

All the DP4+ calculations were carried out using a home-made Matlab script.

\section{References:}

1) Frisch, M. J.; Trucks, G. W.; Schlegel, H. B.; Scuseria, G. E.; Robb, M. A.; Cheeseman, J. R.; Scalmani, G.; Barone, V.; Mennucci, B.; Petersson, G. A.; Nakatsuji, H.; Caricato, M.; Li, X.; Hratchian, H. P.; Izmaylov, A. F.; Bloino, J.; Zheng, G.; Sonnenberg, J. L.; Hada, M.; Ehara, M.; Toyota, K.; Fukuda, R.; Hasegawa, J.; Ishida, M.; Nakajima, T.; Honda, Y.; Kitao, O.; Nakai, H.; Vreven, T.; Montgomery, J. A., Jr., Peralta, J. E.; Ogliaro, F.; Bearpark, M.; Heyd, J. J.; Brothers, E.; Kudin, K. N.; Staroverov, V. N.; Kobayashi, R.; Normand, J.; Raghavachari, K.; Rendell, A.; Burant, J. C.; Iyengar, S. S.; Tomasi, J.; Cossi, M.; Rega, N.; Millam, J. M.; Klene, M.; Knox, J. E.; Cross, J. B.; Bakken, V.; Adamo, C.; Jaramillo, J.; Gomperts, R.; Stratmann, R. E.; Yazyev, O.; Austin, A. J.; Cammi, R.; Pomelli, C.; Ochterski, J. W.; Martin, R. L.; Morokuma, K.; Zakrzewski, V. G.; Voth, G. A.; Salvador, P.; Dannenberg, J. J.; Dapprich, S.; Daniels, A. D.; Farkas, O.; Foresman, J. B.; Ortiz, J. V.; Cioslowski, J.; Fox, D. J. Gaussian 09, Gaussian, Inc.: Wallingford, CT, 2009.

2) MacroModel Schrodinger release 2018-3; Schrodinger LLC: New York, 2018. 


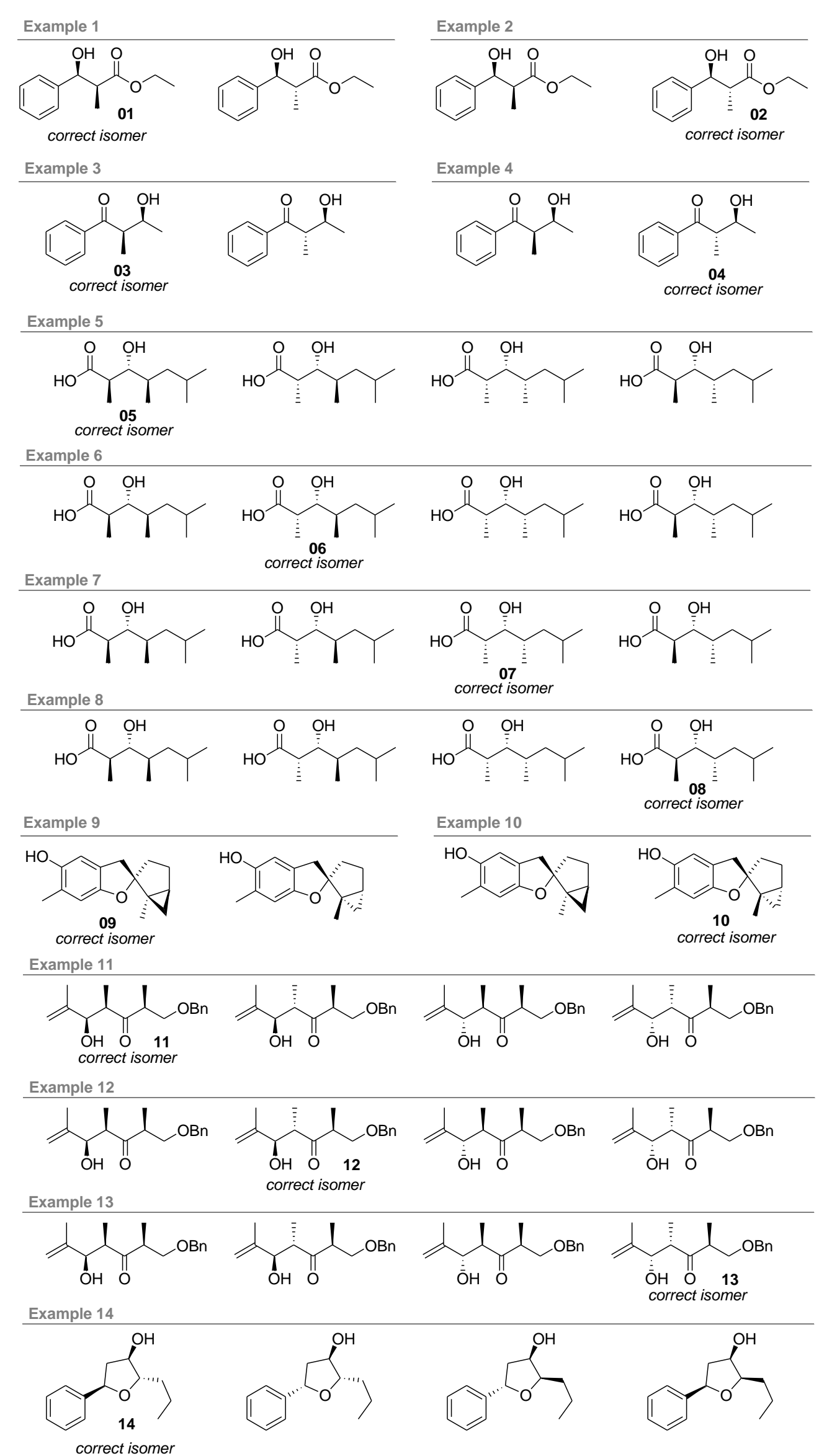

S-12 


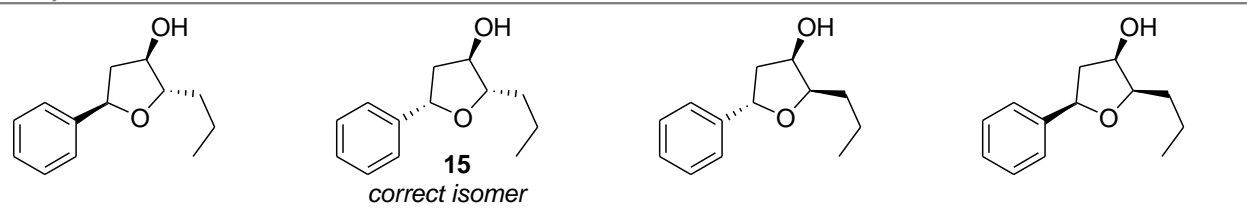

Example 16

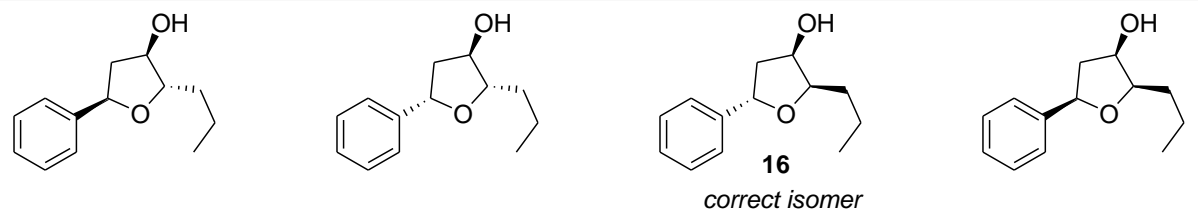

Example 17
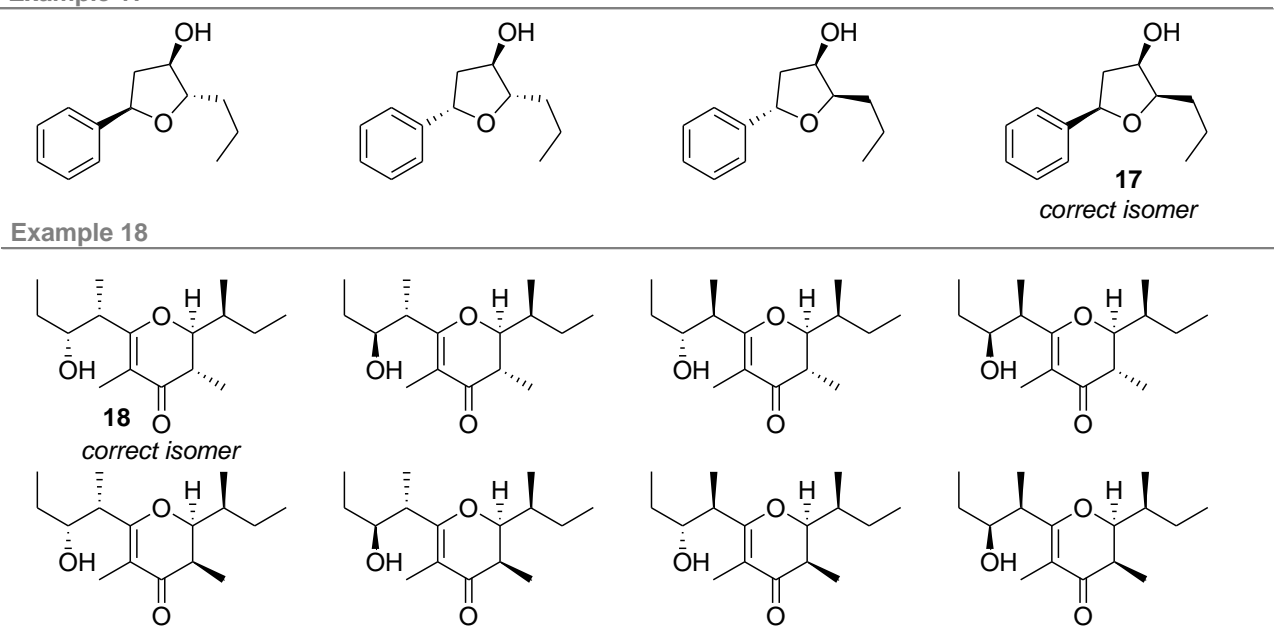

Example 19

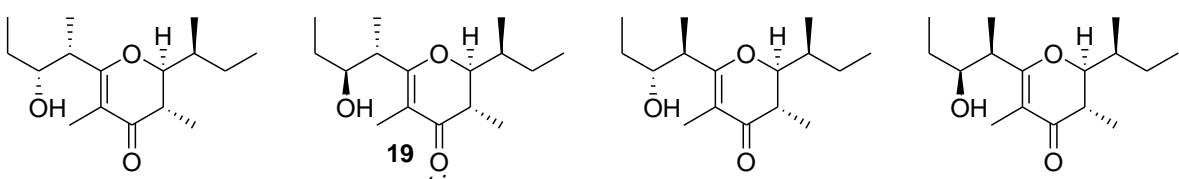

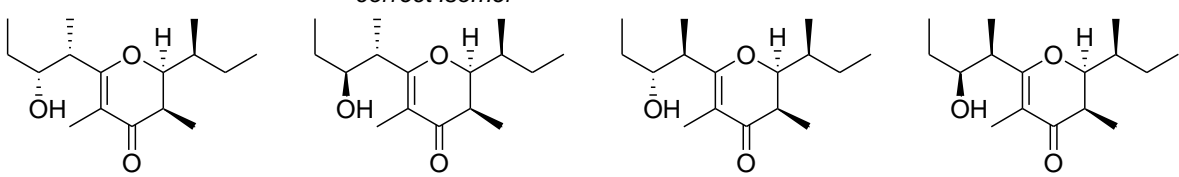

Example 20

年)

$\underbrace{O}_{0}$

(correct isomer

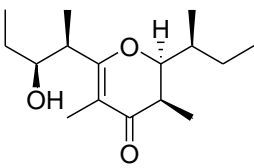

Example 21

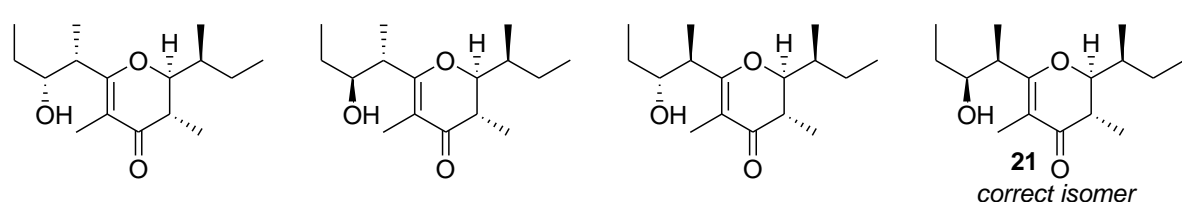

$\underbrace{O}_{0}$

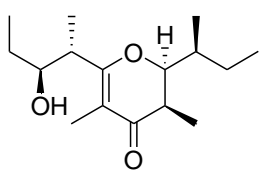

$\underbrace{(1)}_{O}$

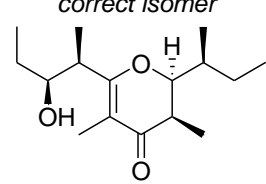




$$
\text { (a)ectisomer }
$$

Example 23

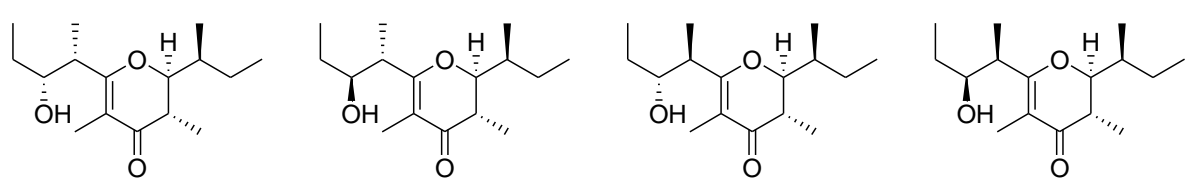

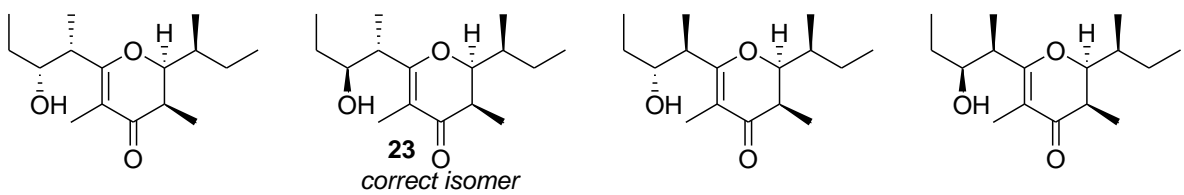

Example 24

(n)

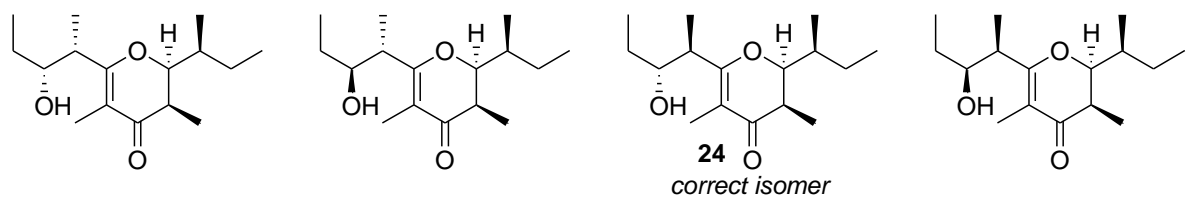

Example 25

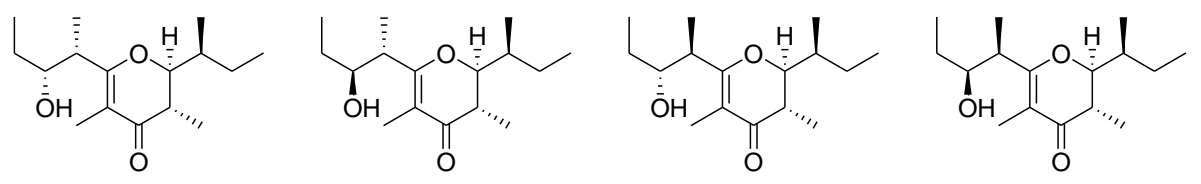

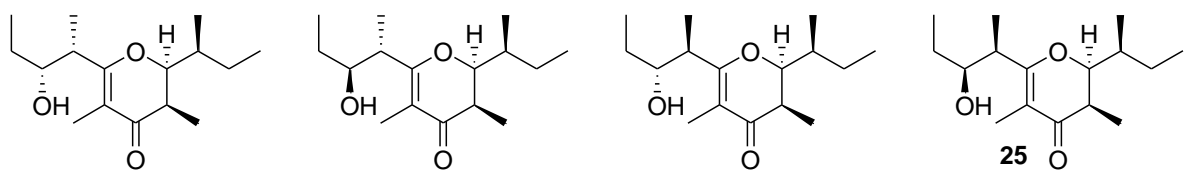

Example 26

Example 27

$$
\overbrace{26}^{\mathrm{OH}}
$$<smiles>CC(C)(C)[C@@H](O)CC(O)C[C@@H](O)C(C)(C)C</smiles>

correct isomer

Example 28

Example 29

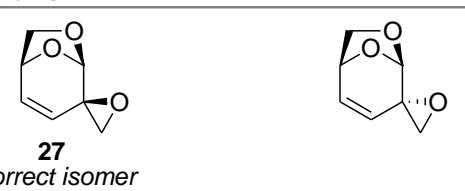

Example 30

Example 31
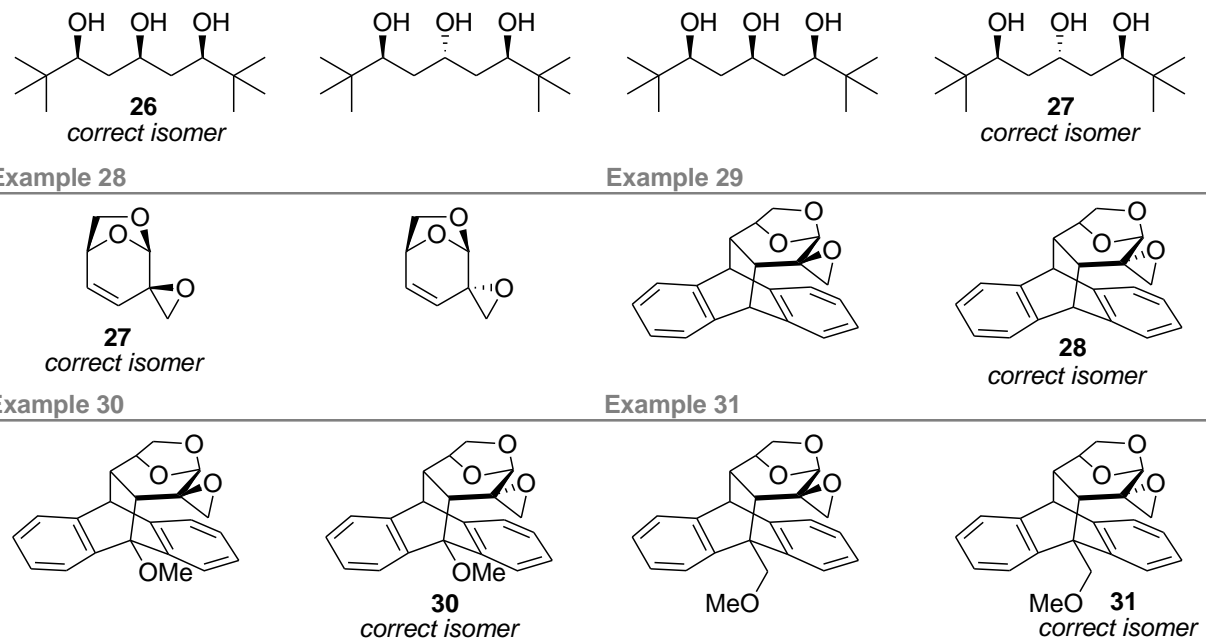


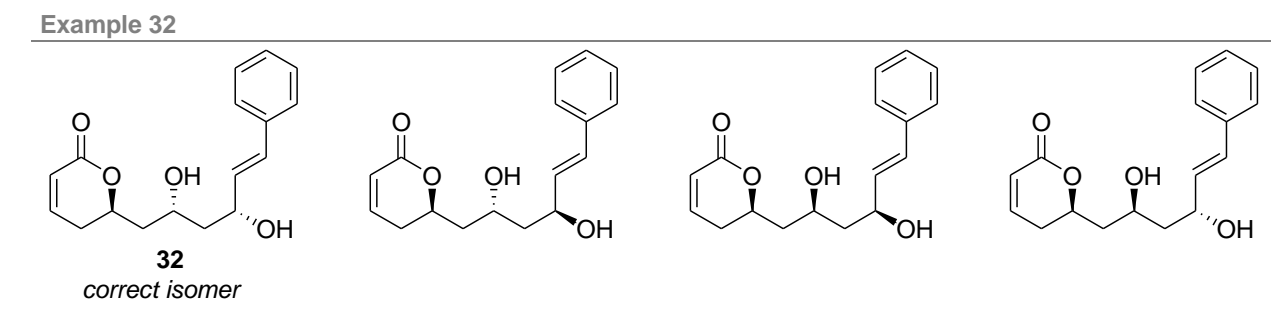

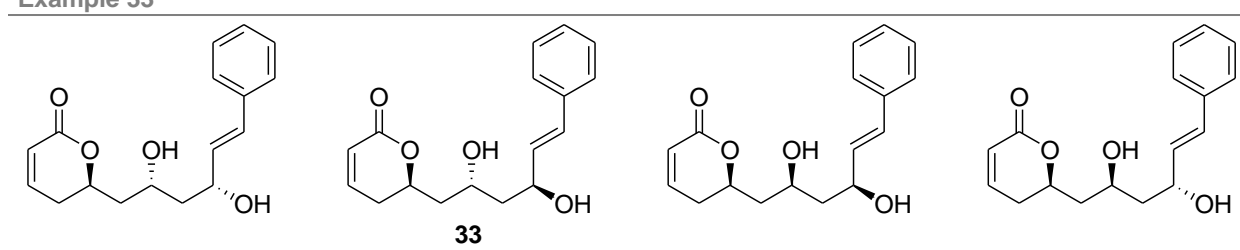

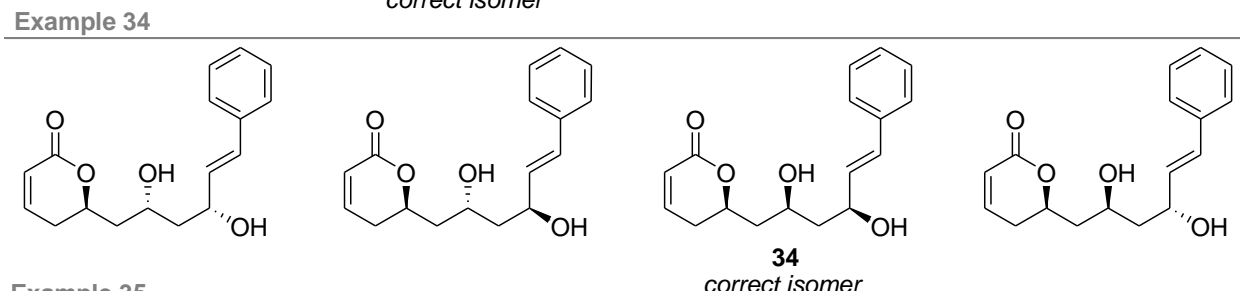

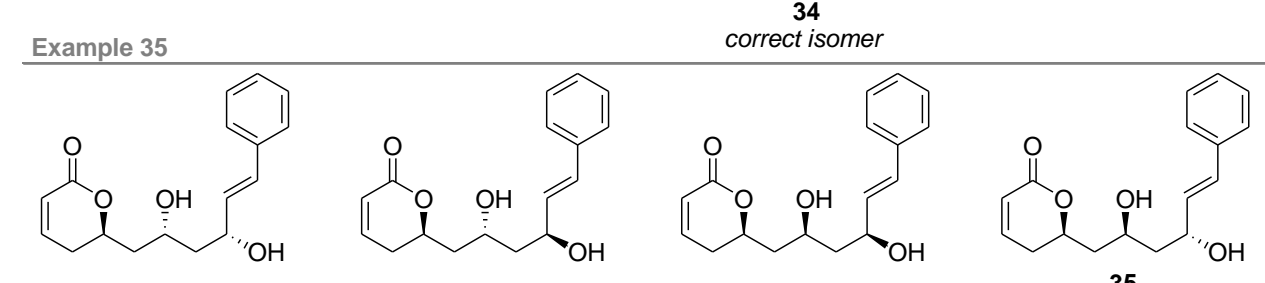

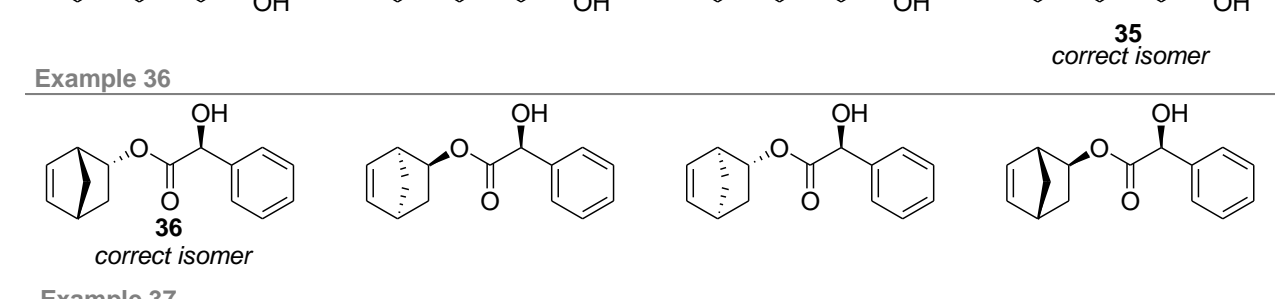

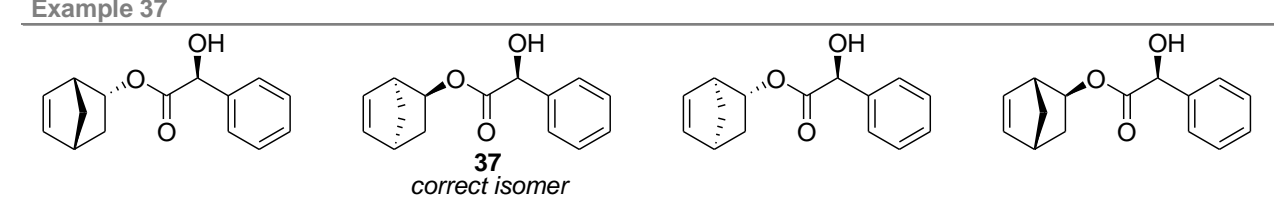

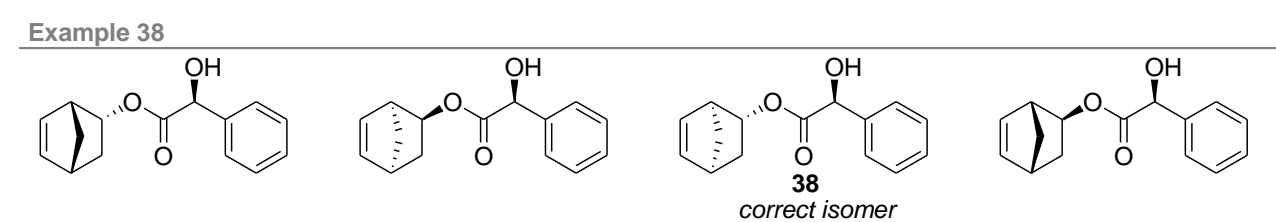

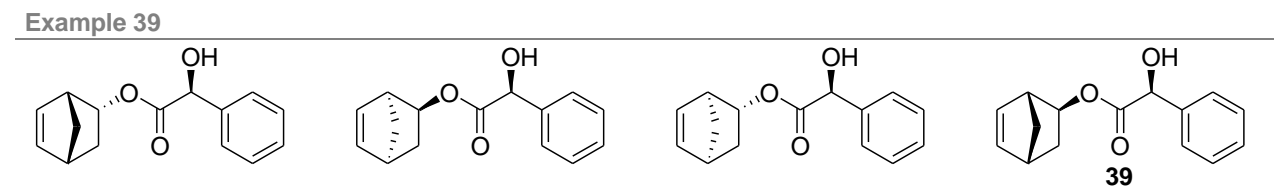

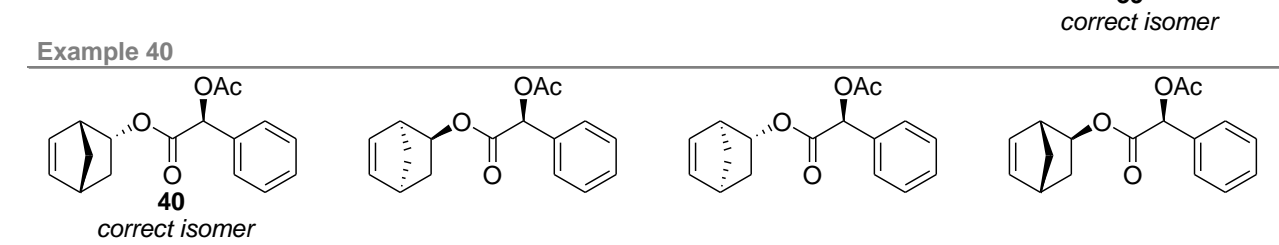

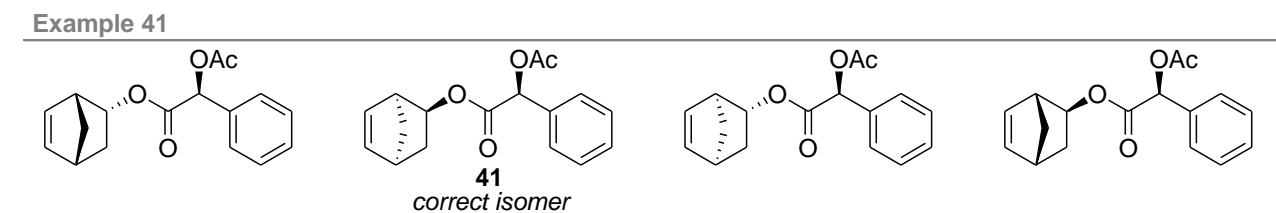

s-15 


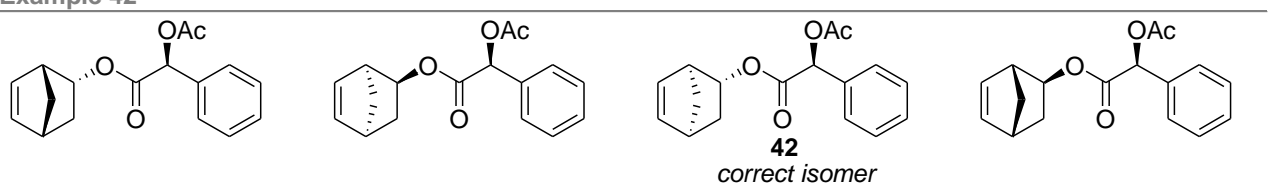

Example 43
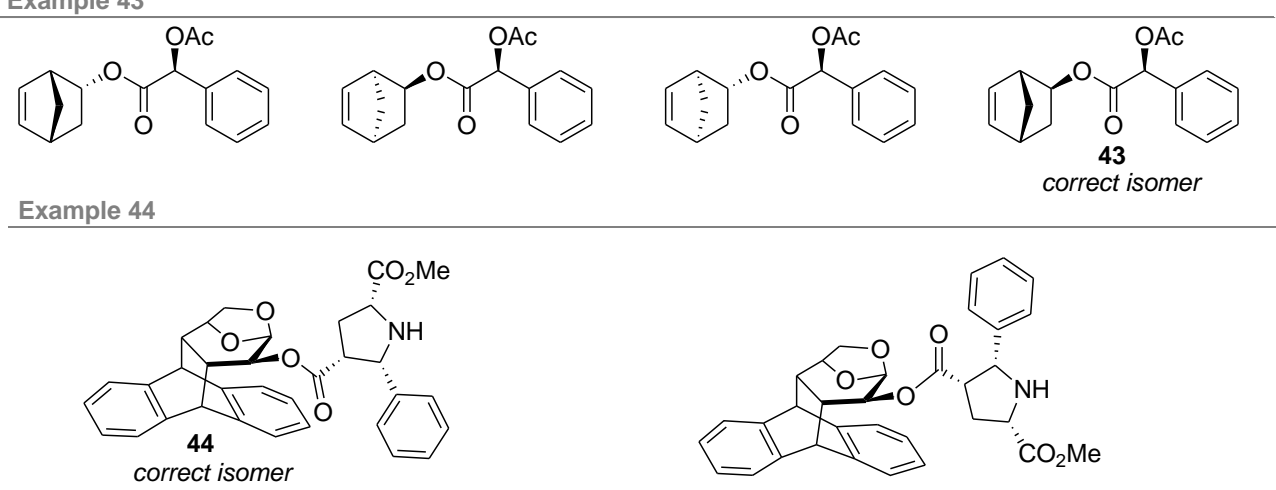

Example 45

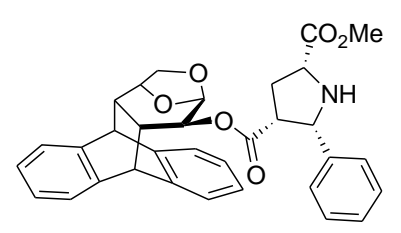

Example 46

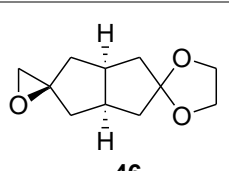

46

correct isomer

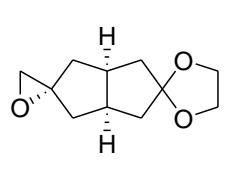

$\bar{H}$
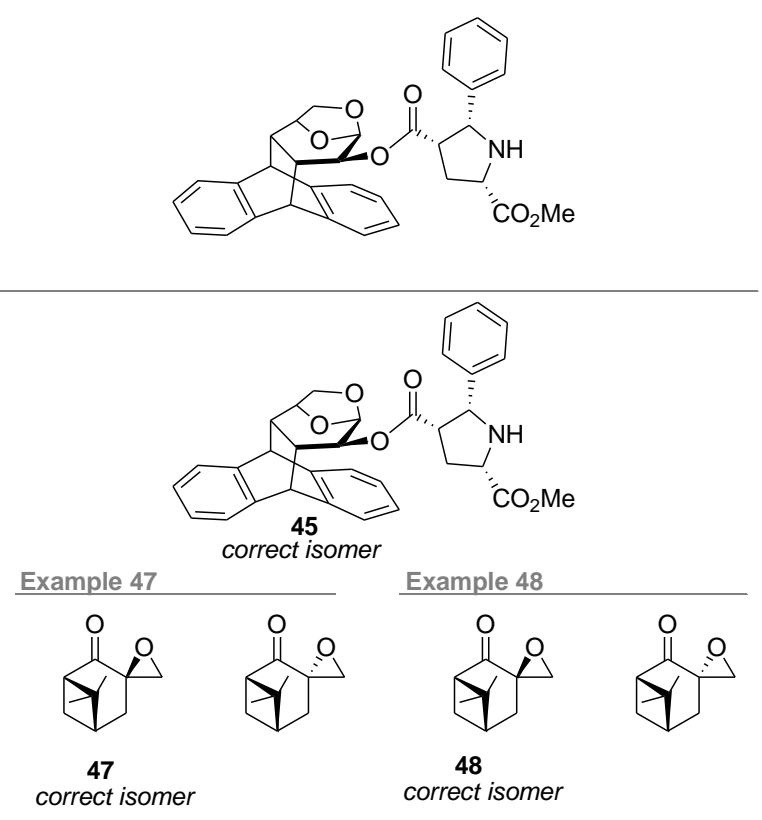
Table S1. $[\mu, \sigma, v]$ parameters estimated at the 24 levels evaluated in the original publication (J. Org. Chem. 2015, 80, 12526) using a set of $77 \mathrm{known}$ organic molecules.

\begin{tabular}{|c|c|c|c|c|c|c|c|c|}
\hline \multirow{2}{*}{ Level of theory (code used in Figure 3a) } & \multicolumn{8}{|c|}{${ }^{13} \mathrm{C}$ NMR data } \\
\hline & $\sigma_{\mathrm{s}}$ & $\mathbf{v}_{\mathbf{s}}$ & $\mu_{\mathrm{u}-\mathrm{sp} 2}$ & $\sigma_{\mathrm{u}-\mathrm{sp} 2}$ & $\mathbf{v}_{\mathrm{u}-\mathrm{sp} 2}$ & $\mu_{\mathrm{u}-\mathrm{sp} 3}$ & $\sigma_{\mathrm{u}-\mathrm{sp} 3}$ & $\mathbf{v}_{\mathrm{u}-\mathrm{sp} 3}$ \\
\hline B3LYP/6-31G*//B3LYP/6-31G* (B1V) & 1.581 & 5.97 & -6.157 & 2.488 & 6.532 & 1.302 & 1.648 & 6.289 \\
\hline B3LYP/6-31G**//B3LYP/6-31G* (B2V) & 1.852 & 8.736 & -4.616 & 2.455 & 6.714 & 2.047 & 1.765 & 7.316 \\
\hline B3LYP/6-31+G**//B3LYP/6-31G* (B3V) & 2.087 & 10.386 & -2.682 & 2.294 & 8.049 & 3.584 & 1.899 & 7.184 \\
\hline B3LYP/6-311G*//B3LYP/6-31G* (B4V) & 1.921 & 7.164 & 5.918 & 2.349 & 5.859 & 4.733 & 2.285 & 15.728 \\
\hline B3LYP/6-311G**//B3LYP/6-31G* (B5V) & 1.837 & 6.341 & 6.538 & 2.241 & 5.026 & 5.265 & 2.212 & 14.147 \\
\hline B3LYP/6-311+G**//B3LYP/6-31G* (B6V) & 1.833 & 7.386 & 7.371 & 2.252 & 6.189 & 5.157 & 2.294 & 18.584 \\
\hline PCM/B3LYP/6-31G*//B3LYP/6-31G* (B1P) & 1.525 & 6.087 & -5.226 & 1.838 & 4.625 & 1.596 & 1.594 & 6.065 \\
\hline PCM/B3LYP/6-31G**//B3LYP/6-31G* (B2P) & 1.784 & 8.777 & -3.696 & 1.837 & 4.86 & 2.336 & 1.752 & 7.415 \\
\hline PCM/B3LYP/6-31+G**//B3LYP/6-31G* (B3P) & 2.034 & 10.172 & -1.417 & 1.994 & 6.562 & 4.044 & 1.895 & 7.359 \\
\hline PCM/B3LYP/6-311G*//B3LYP/6-31G* (B4P) & 1.841 & 7.315 & 7.279 & 2.647 & 14.742 & 5.165 & 2.355 & 20.697 \\
\hline PCM/B3LYP/6-311G**//B3LYP/6-31G* (B5 P) & 1.738 & 6.366 & 7.809 & 2.409 & 8.781 & 5.678 & 2.271 & 17.407 \\
\hline $\mathrm{PCM} / \mathrm{B} 3 \mathrm{LYP} / 6-311+\mathrm{G}^{* *} / / \mathrm{B} 3 \mathrm{LYP} / 6-31 \mathrm{G}^{*}(\mathrm{~B} 6 \mathrm{P})$ & 1.803 & 7.943 & 8.919 & 3.064 & 77.024 & 5.599 & 2.395 & 23.235 \\
\hline mPW1PW91/6-31G*//B3LYP/6-31G* (M1V) & 1.267 & 4.192 & -4.787 & 2.41 & 7.026 & 0.745 & 1.484 & 5.691 \\
\hline mPW1PW91/6-31G**//B3LYP/6-31G* (M2V) & 1.446 & 5.325 & -3.346 & 2.304 & 6.531 & 1.41 & 1.516 & 6.07 \\
\hline mPW1PW91/6-31+G**//B3LYP/6-31G* (M3V) & 1.598 & 6.113 & -2.185 & 2.105 & 6.551 & 2.431 & 1.605 & 6.039 \\
\hline mPW1PW91/6-311G*//B3LYP/6-31G* (M4V) & 1.603 & 4.905 & 6.084 & 2.04 & 4.276 & 4.284 & 1.74 & 6.303 \\
\hline mPW1PW91/6-311G**//B3LYP/6-31G* (M5V) & 1.547 & 4.484 & 6.781 & 2.048 & 4.122 & 4.851 & 1.689 & 6.178 \\
\hline mPW1PW91/6-311+G**//B3LYP/6-31G* (M6V) & 1.582 & 5.761 & 7.515 & 1.965 & 4.839 & 4.748 & 1.814 & 8.744 \\
\hline PCM/mPW1PW91/6-31G*//B3LYP/6-31G*(M1P) & 1.227 & 4.431 & -3.787 & 1.724 & 4.484 & 1.087 & 1.433 & 5.558 \\
\hline PCM/mPW1PW91/6-31G**//B3LYP/6-31G* (M2P) & 1.391 & 5.576 & -2.365 & 1.675 & 4.673 & 1.742 & 1.508 & 6.216 \\
\hline $\mathrm{PCM} / \mathrm{mPW} 1 \mathrm{PW} 91 / 6-31+\mathrm{G}^{* *} / / \mathrm{B} 3 \mathrm{LYP} / 6-31 \mathrm{G}^{*}$ (M3P) & 1.557 & 6.227 & -0.92 & 1.748 & 5.364 & 2.909 & 1.6 & 6.269 \\
\hline PCM/mPW1PW91/6-311G*//B3LYP/6-31G* (M4P) & 1.56 & 5.798 & 6.321 & 2.31 & 9.605 & 3.641 & 1.858 & 9.397 \\
\hline $\mathrm{PCM} / \mathrm{mPW} 1 \mathrm{PW} 91 / 6-311 \mathrm{G}^{* * / / B 3 L Y P / 6-31 G *}(\mathrm{M} 5 \mathrm{P})$ & 1.491 & 5.226 & 6.885 & 2.144 & 6.663 & 4.154 & 1.787 & 8.592 \\
\hline PCM/mPW1PW91/6-311+G**//B3LYP/6-31G* (M6P) & 1.575 & 6.671 & 7.911 & 2.702 & 46.563 & 4.072 & 1.935 & 11.156 \\
\hline Minimum & 1.23 & 4.19 & -6.16 & 1.68 & 4.12 & 0.75 & 1.43 & 5.56 \\
\hline Maximum & 2.09 & 10.39 & 8.92 & 3.06 & 77.02 & 5.68 & 2.40 & 23.24 \\
\hline$R M S$ & 0.22 & 1.67 & 5.53 & 0.34 & 16.41 & 1.60 & 0.30 & 5.35 \\
\hline
\end{tabular}

\begin{tabular}{|c|c|c|c|c|c|c|c|c|}
\hline \multirow{2}{*}{ Level of theory (code used in Figure 3a) } & \multicolumn{8}{|c|}{${ }^{1} \mathrm{H}$ NMR data } \\
\hline & $\sigma_{\mathrm{s}}$ & $\mathbf{v}_{\mathrm{s}}$ & $\mu_{\mathrm{u}-\mathrm{sp} 2}$ & $\sigma_{\mathrm{u}-\mathrm{sp} 2}$ & $v_{\mathrm{u}-\mathrm{sp} 2}$ & $\mu_{\mathrm{u}-\mathrm{sp} 3}$ & $\sigma_{\mathrm{u}-\mathrm{sp} 3}$ & $v_{\mathrm{u}-\mathrm{sp} 3}$ \\
\hline B3LYP/6-31G*//B3LYP/6-31G* (B1V) & 0.140 & 5.942 & -0.172 & 0.194 & 13.808 & -0.056 & 0.169 & 6.950 \\
\hline B3LYP/6-31G**//B3LYP/6-31G* (B2V) & 0.128 & 4.811 & 0.016 & 0.177 & 9.198 & -0.072 & 0.149 & 4.828 \\
\hline B3LYP/6-31+G**//B3LYP/6-31G* (B3V) & 0.127 & 4.703 & 0.139 & 0.163 & 6.839 & -0.06 & 0.134 & 4.047 \\
\hline B3LYP/6-311G*//B3LYP/6-31G* (B4V) & 0.116 & 3.295 & -0.035 & 0.169 & 10.935 & -0.029 & 0.149 & 3.925 \\
\hline B3LYP/6-311G**//B3LYP/6-31G* (B5V) & 0.113 & 3.197 & 0.112 & 0.149 & 7.274 & -0.072 & 0.13 & 3.342 \\
\hline B3LYP/6-311+G**//B3LYP/6-31G* (B6V) & 0.119 & 3.409 & 0.163 & 0.145 & 5.785 & -0.051 & 0.135 & 3.531 \\
\hline PCM/B3LYP/6-31G*//B3LYP/6-31G* (B1P) & 0.129 & 6.408 & -0.091 & 0.172 & 10.337 & -0.013 & 0.151 & 7.441 \\
\hline PCM/B3LYP/6-31G**//B3LYP/6-31G* (B2P) & 0.115 & 4.897 & 0.097 & 0.149 & 7.268 & -0.027 & 0.13 & 4.715 \\
\hline PCM/B3LYP/6-31+G**//B3LYP/6-31G* (B3P) & 0.110 & 4.273 & 0.238 & 0.132 & 5.347 & -0.003 & 0.119 & 3.972 \\
\hline PCM/B3LYP/6-311G*//B3LYP/6-31G* (B4P) & 0.103 & 3.228 & 0.063 & 0.148 & 8.854 & 0.019 & 0.126 & 3.466 \\
\hline PCM/B3LYP/6-311G**//B3LYP/6-31G* (B5 P) & 0.095 & 2.850 & 0.206 & 0.124 & 5.987 & -0.022 & 0.105 & 2.756 \\
\hline $\mathrm{PCM} / \mathrm{B} 3 \mathrm{LYP} / 6-311+\mathrm{G}^{* *} / / \mathrm{B} 3 \mathrm{LYP} / 6-31 \mathrm{G}^{*}(\mathrm{~B} 6 \mathrm{P})$ & 0.099 & 2.929 & 0.267 & 0.119 & 5.196 & 0.008 & 0.115 & 3.033 \\
\hline mPW1PW91/6-31G*//B3LYP/6-31G* (M1V) & 0.128 & 5.030 & -0.044 & 0.185 & 13.042 & -0.065 & 0.156 & 6.129 \\
\hline mPW1PW91/6-31G**//B3LYP/6-31G* (M2V) & 0.120 & 4.303 & 0.14 & 0.167 & 8.595 & -0.085 & 0.136 & 4.223 \\
\hline mPW1PW91/6-31+G**//B3LYP/6-31G* (M3V) & 0.122 & 4.318 & 0.239 & 0.149 & 5.840 & -0.075 & 0.127 & 3.684 \\
\hline mPW1PW91/6-311G*//B3LYP/6-31G* (M4V) & 0.113 & 3.160 & 0.305 & 0.168 & 10.205 & 0.239 & 0.148 & 3.878 \\
\hline mPW1PW91/6-311G**//B3LYP/6-31G* (M5V) & 0.110 & 3.061 & 0.478 & 0.142 & 6.250 & 0.193 & 0.123 & 3.124 \\
\hline mPW1PW91/6-311+G**//B3LYP/6-31G* (M6V) & 0.117 & 3.394 & 0.516 & 0.138 & 5.394 & 0.209 & 0.131 & 3.388 \\
\hline PCM/mPW1PW91/6-31G*//B3LYP/6-31G*(M1P) & 0.117 & 5.543 & 0.047 & 0.162 & 10.576 & -0.021 & 0.136 & 6.259 \\
\hline PCM/mPW1PW91/6-31G**//B3LYP/6-31G* (M2P) & 0.105 & 4.323 & 0.233 & 0.136 & 6.954 & -0.038 & 0.116 & 4.026 \\
\hline $\mathrm{PCM} / \mathrm{mPW} 1 \mathrm{PW} 91 / 6-31+\mathrm{G}^{* * / / B} 3 \mathrm{LYP} / 6-31 \mathrm{G}^{*}$ (M3P) & 0.104 & 3.893 & 0.347 & 0.118 & 4.911 & -0.018 & 0.112 & 3.651 \\
\hline PCM/mPW1PW91/6-311G*//B3LYP/6-31G* (M4P) & 0.101 & 3.157 & 0.133 & 0.147 & 8.764 & 0.009 & 0.125 & 3.536 \\
\hline PCM/mPW1PW91/6-311G**//B3LYP/6-31G* (M5P) & 0.093 & 2.784 & 0.297 & 0.117 & 5.315 & -0.039 & 0.098 & 2.599 \\
\hline PCM/mPW1PW91/6-311+G**//B3LYP/6-31G* (M6P) & 0.098 & 2.891 & 0.344 & 0.112 & 4.880 & -0.014 & 0.111 & 2.891 \\
\hline Minimum & 0.093 & 2.784 & -0.172 & 0.112 & 4.880 & -0.085 & 0.098 & 2.599 \\
\hline Maximum & 0.140 & 6.408 & 0.516 & 0.194 & 13.808 & 0.239 & 0.169 & 7.441 \\
\hline$R M S$ & 0.012 & 1.048 & 0.171 & 0.022 & 2.599 & 0.089 & 0.017 & 1.305 \\
\hline
\end{tabular}


Table S2. $[\mu, \sigma]$ parameters estimated at 28 new levels using the reduced set of 8 molecules shown in Figure 5.

\begin{tabular}{|c|c|c|c|c|c|c|c|c|c|c|c|}
\hline \multirow{2}{*}{ Entry } & \multirow{2}{*}{ Level of theory } & \multicolumn{5}{|c|}{${ }^{13} \mathrm{C}$ NMR data } & \multicolumn{5}{|c|}{${ }^{1}$ H NMR data } \\
\hline & & $\mu_{\mathrm{u}-\mathrm{sp} 2}$ & $\sigma_{\mathrm{u}-\mathrm{sp} 2}$ & $\mu_{\mathrm{u}-\mathrm{sp} 3}$ & $\sigma_{\mathrm{u}-\mathrm{sp} 3}$ & $\sigma_{\mathrm{s}}$ & $\mu_{\mathrm{u}-\mathrm{sp} 2}$ & $\sigma_{\mathrm{u}-\mathrm{sp} 2}$ & $\mu_{\mathrm{u}-\mathrm{sp} 3}$ & $\sigma_{\mathrm{u}-\mathrm{sp} 3}$ & $\sigma_{\mathrm{s}}$ \\
\hline 1 & PCM/mPW1PW91/6-31+G**//MMFF & 0.64 & 3.47 & 3.50 & 1.64 & 2.28 & 0.48 & 0.14 & 0.08 & 0.12 & 0.11 \\
\hline 2 & $\mathrm{PCM} / \mathrm{mPW} 1 \mathrm{PW} 91 / 6-31+\mathrm{G} * * / / \mathrm{HF} / 3-21 \mathrm{G}$ & -1.48 & 2.11 & 3.45 & 2.15 & 2.39 & 0.34 & 0.16 & -0.10 & 0.15 & 0.12 \\
\hline 3 & PCM/mPW1PW91/6-31+G**//B3LYP/6-31G* & -0.89 & 2.07 & 2.94 & 1.53 & 1.77 & 0.34 & 0.13 & -0.02 & 0.12 & 0.10 \\
\hline 4 & $\mathrm{PCM} / \mathrm{mPW} 1 \mathrm{PW} 91 / 6-31+\mathrm{G}^{* *} / / \mathrm{M} 06-2 \mathrm{X} / 6-31 \mathrm{G}^{*}$ & -1.45 & 2.12 & 2.57 & 1.51 & 1.61 & 0.36 & 0.14 & -0.01 & 0.13 & 0.11 \\
\hline 5 & $\mathrm{PCM} / \mathrm{mPW} 1 \mathrm{PW} 91 / 6-31+\mathrm{G}^{* * / / w B 97 X D / 6-31 G *}$ & -1.65 & 2.13 & 2.40 & 1.50 & 1.62 & 0.33 & 0.14 & -0.04 & 0.13 & 0.11 \\
\hline 6 & $\mathrm{PCM} / \mathrm{mPW} 1 \mathrm{PW} 91 / 6-31+\mathrm{G}^{* *} / / \mathrm{PCM} / \mathrm{B} 3 \mathrm{LYP} / 6-311+\mathrm{G}^{* *}$ & -0.75 & 2.04 & 3.03 & 1.49 & 1.80 & 0.37 & 0.13 & -0.02 & 0.12 & 0.10 \\
\hline 7 & $\mathrm{PCM} / \mathrm{mPW} 1 \mathrm{PW} 91 / 6-31+\mathrm{G}^{* *} / / \mathrm{SMD} / \mathrm{B} 3 \mathrm{LYP} / 6-311+\mathrm{G}^{* *}$ & 0.52 & 2.44 & 3.71 & 1.66 & 2.10 & 0.45 & 0.13 & -0.02 & 0.13 & 0.11 \\
\hline 8 & 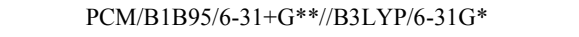 & 2.08 & 2.16 & 3.32 & 1.65 & 1.77 & 0.38 & 0.15 & -0.01 & 0.15 & 0.12 \\
\hline 9 & PCM/B3LYP/6-31+G**//B3LYP/6-31G* & -1.43 & 2.19 & 3.95 & 1.86 & 2.16 & 0.24 & 0.15 & -0.01 & 0.13 & 0.11 \\
\hline 10 & PCM/LC-TPSSTPSS/6-31+G**//B3LYP/6-31G* & 4.70 & 2.09 & 0.27 & 1.27 & 1.53 & 0.48 & 0.16 & -0.07 & 0.10 & 0.11 \\
\hline 11 & PCM/LC-wPBE/6-31+G**//B3LYP/6-31G* & 3.53 & 1.76 & 1.02 & 1.29 & 1.36 & 0.45 & 0.15 & -0.08 & 0.11 & 0.11 \\
\hline 12 & PCM/M06-2X/6-31+G**//B3LYP/6-31G* & 14.75 & 3.40 & 4.86 & 2.11 & 2.24 & 0.81 & 0.19 & -0.03 & 0.18 & 0.15 \\
\hline 13 & PCM/PBE1PBE/6-31+G**//B3LYP/6-31G* & -0.86 & 2.08 & 2.88 & 1.52 & 1.75 & 0.35 & 0.14 & -0.02 & 0.12 & 0.10 \\
\hline 14 & PCM/VSXC/6-31+G**//B3LYP/6-31G* & -8.51 & 3.83 & 7.26 & 4.07 & 4.50 & 0.10 & 0.42 & 0.14 & 0.39 & 0.35 \\
\hline 15 & $\mathrm{PCM} / \mathrm{wB} 97 \mathrm{XD} / 6-31+\mathrm{G}^{* *} / / \mathrm{B} 3 \mathrm{LYP} / 6-31 \mathrm{G}^{*}$ & 0.17 & 1.80 & 2.50 & 1.57 & 1.64 & 0.37 & 0.15 & -0.06 & 0.14 & 0.12 \\
\hline 16 & PCM/X3LYP/6-31+G**//B3LYP/6-31G* & -1.15 & 2.15 & 3.86 & 1.84 & 2.11 & 0.25 & 0.15 & -0.01 & 0.13 & 0.11 \\
\hline 17 & PCM/mPW1PW91/6-31G*//B3LYP/6-31G* & -3.62 & 2.14 & 1.30 & 1.23 & 1.39 & 0.04 & 0.16 & -0.03 & 0.12 & 0.11 \\
\hline 18 & PCM/mPW1PW91/6-311+G**//B3LYP/6-31G* & 7.93 & 2.50 & 4.10 & 1.96 & 1.78 & 0.34 & 0.12 & -0.01 & 0.11 & 0.09 \\
\hline 19 & $\mathrm{PCM} / \mathrm{mPW} 1 \mathrm{PW} 91 / 6-311++\mathrm{G}(3 \mathrm{df}, 2 \mathrm{dp}) / / \mathrm{B} 3 \mathrm{LYP} / 6-31 \mathrm{G}^{*}$ & 8.59 & 2.47 & 4.68 & 2.07 & 1.76 & 0.34 & 0.12 & -0.02 & 0.09 & 0.08 \\
\hline 20 & PCM/mPW1PW91/cc-PVDZ//B3LYP/6-31G* & 1.92 & 1.97 & 3.37 & 1.85 & 1.78 & 0.09 & 0.15 & -0.16 & 0.12 & 0.09 \\
\hline 21 & PCM/mPW1PW91/cc-PVTZ//B3LYP/6-31G* & 7.55 & 2.16 & 4.17 & 2.08 & 1.68 & 0.31 & 0.12 & -0.02 & 0.09 & 0.07 \\
\hline 22 & PCM/mPW1PW91/def2-SVP//B3LYP/6-31G* & 0.96 & 1.80 & 1.53 & 1.34 & 1.34 & 0.02 & 0.15 & -0.23 & 0.12 & 0.10 \\
\hline 23 & PCM/mPW1PW91/def2-TZVPP//B3LYP/6-31G* & 8.70 & 2.43 & 4.57 & 2.25 & 1.75 & 0.34 & 0.11 & -0.02 & 0.09 & 0.07 \\
\hline 24 & PCM/mPW1PW91/PCS2//B3LYP/6-31G* & 14.75 & 3.03 & 6.92 & 2.73 & 1.76 & 0.38 & 0.11 & 0.00 & 0.09 & 0.07 \\
\hline 25 & mPW1PW91/6-31+G**//B3LYP/6-31G* & -2.31 & 2.29 & 2.47 & 1.49 & 1.64 & 0.24 & 0.16 & -0.08 & 0.14 & 0.12 \\
\hline 26 & SMD/mPW1PW91/6-31+G**//B3LYP/6-31G* & 0.34 & 2.38 & 3.63 & 1.68 & 2.02 & 0.44 & 0.14 & 0.00 & 0.14 & 0.11 \\
\hline 27 & CPCM/mPW1PW91/6-31+G**//B3LYP/6-31G* & -0.70 & 2.11 & 3.03 & 1.54 & 1.80 & 0.36 & 0.13 & -0.01 & 0.12 & 0.10 \\
\hline \multirow[t]{4}{*}{28} & CSGT//PCM/mPW1PW91/6-31+G**//B3LYP/6-31G* & 5.08 & 2.10 & 4.58 & 1.54 & 1.65 & -1.53 & 0.28 & -1.07 & 0.26 & 0.19 \\
\hline & Minimum & -8.51 & 1.76 & 0.27 & 1.23 & 1.34 & -1.53 & 0.11 & -1.07 & 0.09 & 0.07 \\
\hline & Maximum & 14.75 & 3.83 & 7.26 & 4.07 & 4.50 & 0.81 & 0.42 & 0.14 & 0.39 & 0.35 \\
\hline & $R M S$ & 5.31 & 0.51 & 1.53 & 0.56 & 0.58 & 0.38 & 0.06 & 0.21 & 0.06 & 0.05 \\
\hline
\end{tabular}


Table S3. Changes in the DP4+ values calculated for the test set (Figure 2) when correlating the GIAO NMR chemical shifts computed at the $\mathrm{PCM} / \mathrm{mPW} 1 \mathrm{PW} 91 / 6-31+\mathrm{G}^{* *} / \mathrm{B} 3 \mathrm{LYP} / 6-31 \mathrm{G}^{*}$ level of theory, with the $[\mu, \sigma, v]$ parameters corresponding to different levels of theory.
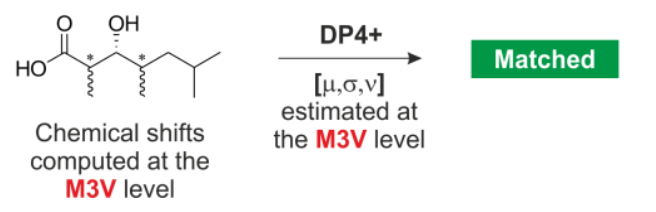

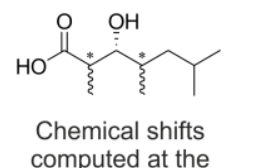

M3V level

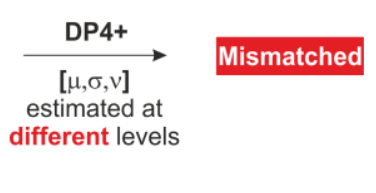

\begin{tabular}{|c|c|c|c|c|}
\hline $\mathbf{N}^{\mathbf{o}}$ & Level of theory of the $[\mu, \sigma, v] \operatorname{set}^{a}$ & $\begin{array}{l}\text { Aver. } \\
\text { DP4+ }\end{array}$ & $\begin{array}{c}\mathbf{N}^{o} \\
\text { Hits }\end{array}$ & Score $^{b}$ \\
\hline 1 & B3LYP/6-31G* (B1V) & 81.0 & 40 & 104 \\
\hline 2 & B3LYP/6-31G** (B2V) & 84.3 & 40 & 108 \\
\hline 3 & B3LYP/6-31+G** (B3V) & 81.7 & 40 & 100 \\
\hline 4 & B3LYP/6-311G* (B4V) & 71.9 & 33 & 87 \\
\hline 5 & B3LYP/6-311G**(B5V) & 70.7 & 34 & 92 \\
\hline 6 & B3LYP/6-311+G** (B6V) & 72.9 & 35 & 91 \\
\hline 7 & PCM/B3LYP/6-31G* (B1P) & 82.8 & 41 & 107 \\
\hline 8 & PCM/B3LYP/6-31G**(B2P) & 85.6 & 41 & 107 \\
\hline 9 & $\mathrm{PCM} / \mathrm{B} 3 \mathrm{LYP} / 6-31+\mathrm{G}^{* *}(\mathrm{~B} 3 \mathrm{P})$ & 79.8 & 38 & 100 \\
\hline 10 & PCM/B3LYP/6-311G* (B4P) & 70.7 & 35 & 91 \\
\hline 11 & PCM/B3LYP/6-311G** (B5 P) & 70.4 & 33 & 91 \\
\hline 12 & $\mathrm{PCM} / \mathrm{B} 3 \mathrm{LYP} / 6-311+\mathrm{G} * *(\mathrm{~B} 6 \mathrm{P})$ & 74.3 & 36 & 94 \\
\hline 13 & mPW1PW91/6-31G* (M1V) & 78.4 & 39 & 103 \\
\hline 14 & mPW1PW91/6-31G** (M2V) & 81.5 & 39 & 107 \\
\hline 15 & mPW1PW91/6-31+G** (M3V) & 85.5 & 42 & 111 \\
\hline 16 & mPW1PW91/6-311G* (M4V) & 69.5 & 33 & 87 \\
\hline 17 & mPW1PW91/6-311G** (M5V) & 72.6 & 37 & 93 \\
\hline 18 & mPW1PW91/6-311+G** (M6V) & 73.0 & 36 & 92 \\
\hline 19 & PCM/mPW1PW91/6-31G* (M1P) & 80.4 & 39 & 105 \\
\hline 20 & $\mathrm{PCM} / \mathrm{mPW} 1 \mathrm{PW} 91 / 6-31 \mathrm{G}^{* *}(\mathrm{M} 2 \mathrm{P})$ & 85.4 & 42 & 110 \\
\hline 21 & $\mathrm{PCM} / \mathrm{mPW} 1 \mathrm{PW} 91 / 6-31+\mathrm{G}^{* *}(\mathrm{M} 3 \mathrm{P})$ & 87.4 & 43 & 112 \\
\hline 22 & PCM/mPW1PW91/6-311G* (M4P) & 77.7 & 37 & 103 \\
\hline 23 & PCM/mPW1PW91/6-311G** (M5P) & 78.1 & 38 & 102 \\
\hline 24 & $\mathrm{PCM} / \mathrm{mPW} 1 \mathrm{PW} 91 / 6-311+\mathrm{G} * *(\mathrm{M} 6 \mathrm{P})$ & 78.9 & 38 & 102 \\
\hline
\end{tabular}

${ }^{a}$ Level of theory for the GIAO NMR calculations at which the $[\mu, \sigma, v]$ parameters were estimated from a full set of 77 molecules using B3LYP/6-31G* optimized geometries in all cases. ${ }^{\mathrm{b}}$ Computed by summing scores from each individual system depending on the DP4+ value of the correct isomer: >95\% (optimal, 3 points); $50 \%-95 \%$ (acceptable, 1 point); and $<50 \%$ (undesirable, 0 point). 
Table S4. Changes in the DP4+ values calculated for the test set (Figure 2) when correlating the GIAO NMR chemical shifts computed at the $\mathrm{PCM} / \mathrm{mPW} 1 \mathrm{PW} 91 / 6-31+\mathrm{G}^{* *} / / \mathrm{B} 3 \mathrm{LYP} / 6-31 \mathrm{G}^{*}$ level of theory $(\mathrm{M} 3 \mathrm{P})$, with the $[\mu, \sigma, v]$ parameters corresponding to different levels of theory. The results obtained with the matchd M3P parameters are shown in grey.

\begin{tabular}{|c|c|c|c|c|c|c|c|c|c|c|c|c|c|}
\hline $\begin{array}{c}\text { Comp } \\
\mathrm{N}^{\mathrm{o}}\end{array}$ & M3P & B1V & B2V & B3V & B4V & B5V & B6V & B1P & B2P & B3P & B4P & B5P & B6P \\
\hline 1 & 100.00 & 100.00 & 100.00 & 100.00 & 99.92 & 99.83 & 99.88 & 100.00 & 100.00 & 100.00 & 99.95 & 99.78 & 99.88 \\
\hline 2 & 97.27 & 95.64 & 98.11 & 98.47 & 99.95 & 99.97 & 99.97 & 96.70 & 97.14 & 98.17 & 99.97 & 99.97 & 99.98 \\
\hline 3 & 100.00 & 100.00 & 100.00 & 99.99 & 99.99 & 99.92 & 99.96 & 100.00 & 100.00 & 99.99 & 100.00 & 99.97 & 99.99 \\
\hline 4 & 99.38 & 30.15 & 96.08 & 99.89 & 99.20 & 99.82 & 99.75 & 52.60 & 97.49 & 99.82 & 98.09 & 99.51 & 98.55 \\
\hline 5 & 93.40 & 76.14 & 85.17 & 94.20 & 94.93 & 96.89 & 96.73 & 76.71 & 90.62 & 95.76 & 95.32 & 97.76 & 96.66 \\
\hline 6 & 100.00 & 100.00 & 100.00 & 99.97 & 99.93 & 99.90 & 99.93 & 100.00 & 100.00 & 99.96 & 99.91 & 99.88 & 99.92 \\
\hline 7 & 98.31 & 99.76 & 98.90 & 89.19 & 71.57 & 60.51 & 58.22 & 99.77 & 98.42 & 94.09 & 84.35 & 65.65 & 84.96 \\
\hline 8 & 100.00 & 99.99 & 99.99 & 99.98 & 99.96 & 99.93 & 99.96 & 100.00 & 100.00 & 99.95 & 99.97 & 99.92 & 99.96 \\
\hline 9 & 100.00 & 100.00 & 100.00 & 100.00 & 100.00 & 100.00 & 100.00 & 100.00 & 100.00 & 100.00 & 100.00 & 100.00 & 100.00 \\
\hline 10 & 100.00 & 100.00 & 100.00 & 100.00 & 100.00 & 100.00 & 100.00 & 100.00 & 100.00 & 100.00 & 100.00 & 100.00 & 100.00 \\
\hline 11 & 100.00 & 100.00 & 100.00 & 99.97 & 48.55 & 54.04 & 85.16 & 100.00 & 100.00 & 99.95 & 7.89 & 31.72 & 83.10 \\
\hline 12 & 100.00 & 100.00 & 100.00 & 100.00 & 100.00 & 99.98 & 99.99 & 100.00 & 100.00 & 100.00 & 100.00 & 100.00 & 100.00 \\
\hline 13 & 99.86 & 98.85 & 99.75 & 99.99 & 71.60 & 58.86 & 88.75 & 99.92 & 99.99 & 99.98 & 53.30 & 65.74 & 99.69 \\
\hline 14 & 99.69 & 99.50 & 99.98 & 99.99 & 99.97 & 100.00 & 100.00 & 99.31 & 99.94 & 99.92 & 99.86 & 99.99 & 99.81 \\
\hline 15 & 96.16 & 6.89 & 38.11 & 92.46 & 47.12 & 55.31 & 63.26 & 7.94 & 43.66 & 97.35 & 47.35 & 63.61 & 71.13 \\
\hline 16 & 100.00 & 100.00 & 100.00 & 100.00 & 100.00 & 100.00 & 100.00 & 100.00 & 100.00 & 100.00 & 100.00 & 100.00 & 100.00 \\
\hline 17 & 100.00 & 100.00 & 100.00 & 100.00 & 100.00 & 100.00 & 100.00 & 100.00 & 100.00 & 100.00 & 100.00 & 100.00 & 100.00 \\
\hline 18 & 36.13 & 56.35 & 35.05 & 2.50 & 0.86 & 0.15 & 0.32 & 77.14 & 59.62 & 4.50 & 1.49 & 0.65 & 0.88 \\
\hline 19 & 19.85 & 2.18 & 8.39 & 5.42 & 22.98 & 14.99 & 20.85 & 14.98 & 19.84 & 15.33 & 63.64 & 32.82 & 58.46 \\
\hline 20 & 49.27 & 34.29 & 46.96 & 62.88 & 48.37 & 41.61 & 41.71 & 26.02 & 44.93 & 48.27 & 14.38 & 32.89 & 16.36 \\
\hline 21 & 80.31 & 91.63 & 96.69 & 48.48 & 3.40 & 5.32 & 4.14 & 82.55 & 89.87 & 14.14 & 1.44 & 3.15 & 1.84 \\
\hline 22 & 92.78 & 99.95 & 99.46 & 48.16 & 0.81 & 0.43 & 0.65 & 99.82 & 97.56 & 10.68 & 0.24 & 0.28 & 0.20 \\
\hline 23 & 73.30 & 92.80 & 85.05 & 8.40 & 6.43 & 2.66 & 3.93 & 97.42 & 87.32 & 12.67 & 14.22 & 5.38 & 12.35 \\
\hline 24 & 25.95 & 6.07 & 14.54 & $\begin{array}{l}0.40 \\
85.69\end{array}$ & 93.28 & 96.01 & 94.98 & 3.38 & 15.54 & 86.57 & 86.81 & 95.64 & 89.79 \\
\hline 25 & 0.00 & 0.00 & 0.00 & 0.00 & 0.00 & 0.00 & 0.00 & 0.00 & 0.00 & 0.00 & 0.00 & 0.00 & 0.00 \\
\hline 26 & 99.99 & 99.61 & 99.95 & 100.00 & 100.00 & 100.00 & 100.00 & 99.86 & 99.98 & 100.00 & 100.00 & 100.00 & 100.00 \\
\hline 27 & 98.23 & 99.87 & 99.49 & 63.44 & 22.20 & 6.51 & 8.07 & 99.72 & 98.67 & 43.55 & 11.98 & 3.01 & 4.60 \\
\hline 28 & 100.00 & 99.98 & 99.99 & 99.99 & 100.00 & 100.00 & 100.00 & 100.00 & 100.00 & 100.00 & 100.00 & 100.00 & 100.00 \\
\hline 29 & 100.00 & 100.00 & 100.00 & 100.00 & 100.00 & 100.00 & 100.00 & 100.00 & 100.00 & 100.00 & 99.99 & 100.00 & 99.98 \\
\hline 30 & 100.00 & 100.00 & 100.00 & 100.00 & 100.00 & 100.00 & 100.00 & 100.00 & 100.00 & 100.00 & 100.00 & 100.00 & 100.00 \\
\hline 31 & 100.00 & 100.00 & 100.00 & 100.00 & 99.99 & 99.97 & 99.98 & 100.00 & 100.00 & 100.00 & 99.98 & 99.96 & 99.96 \\
\hline 32 & 99.30 & 99.65 & 99.89 & 99.26 & 92.76 & 87.17 & 91.56 & 99.56 & 99.82 & 97.59 & 83.76 & 80.89 & 91.07 \\
\hline 33 & 97.79 & 92.95 & 84.55 & 74.96 & 52.78 & 40.73 & 59.96 & 94.68 & 90.16 & 71.99 & 61.02 & 46.59 & 68.38 \\
\hline 34 & 0.34 & 4.06 & 6.99 & 0.49 & 0.06 & 0.03 & 0.03 & 3.13 & 1.51 & 0.01 & 0.00 & 0.00 & 0.00 \\
\hline 35 & 97.81 & 50.08 & 90.66 & 99.52 & 99.82 & 99.87 & 99.83 & 82.53 & 98.36 & 99.82 & 99.95 & 99.94 & 99.88 \\
\hline 36 & 74.69 & 83.78 & 76.70 & 72.32 & 26.05 & 13.10 & 26.93 & 96.62 & 92.14 & 89.78 & 58.20 & 18.50 & 44.89 \\
\hline 37 & 99.98 & 99.98 & 99.96 & 99.98 & 100.00 & 99.99 & 99.99 & 99.99 & 99.99 & 100.00 & 100.00 & 100.00 & 100.00 \\
\hline 38 & 99.99 & 99.99 & 99.99 & 99.99 & 99.96 & 99.85 & 99.94 & 100.00 & 100.00 & 100.00 & 99.99 & 99.97 & 99.99 \\
\hline 39 & 100.00 & 100.00 & 100.00 & 100.00 & 100.00 & 100.00 & 100.00 & 100.00 & 100.00 & 100.00 & 100.00 & 100.00 & 100.00 \\
\hline 40 & 77.80 & 0.32 & 1.00 & 7.61 & 0.38 & 2.18 & 6.45 & 0.39 & 3.21 & 51.05 & 0.78 & 15.26 & 33.37 \\
\hline 41 & 100.00 & 100.00 & 100.00 & 100.00 & 100.00 & 100.00 & 100.00 & 100.00 & 100.00 & 100.00 & 100.00 & 100.00 & 100.00 \\
\hline 42 & 100.00 & 99.99 & 99.99 & 99.99 & 99.97 & 99.92 & 99.96 & 100.00 & 100.00 & 100.00 & 99.99 & 99.99 & 99.98 \\
\hline 43 & 100.00 & 99.99 & 100.00 & 100.00 & 100.00 & 100.00 & 100.00 & 100.00 & 100.00 & 100.00 & 100.00 & 100.00 & 100.00 \\
\hline 44 & 99.96 & 99.94 & 99.93 & 99.99 & 100.00 & 100.00 & 100.00 & 99.99 & 99.99 & 100.00 & 100.00 & 100.00 & 100.00 \\
\hline 45 & 86.39 & 96.99 & 98.23 & 88.92 & 8.75 & 37.70 & 21.30 & 88.69 & 93.28 & 30.82 & 1.20 & 6.81 & 1.06 \\
\hline 46 & 99.82 & 99.98 & 99.93 & 95.32 & 40.86 & 24.66 & 26.39 & 99.94 & 99.85 & 76.01 & 10.59 & 15.83 & 8.18 \\
\hline 47 & 100.00 & 100.00 & 100.00 & 100.00 & 100.00 & 99.99 & 100.00 & 100.00 & 100.00 & 100.00 & 100.00 & 99.99 & 99.99 \\
\hline 48 & 98.66 & 71.00 & 86.74 & 83.42 & 97.96 & 97.60 & 98.41 & 73.69 & 88.26 & 90.89 & 99.32 & 99.06 & 99.64 \\
\hline
\end{tabular}


Table S4 (cont). Changes in the DP4+ values calculated for the test set (Figure 2) when correlating the GIAO NMR chemical shifts computed at the $\mathrm{PCM} / \mathrm{mPW} 1 \mathrm{PW} 91 / 6-31+\mathrm{G}^{* *} / / \mathrm{B} 3 \mathrm{LYP} / 6-31 \mathrm{G}^{*}$ level of theory (M3P), with the $[\mu, \sigma, v]$ parameters corresponding to different levels of theory. The results obtained with the matchd M3P parameters are shown in grey.

\begin{tabular}{|c|c|c|c|c|c|c|c|c|c|c|c|c|c|}
\hline $\begin{array}{c}\text { Comp } \\
\mathrm{N}^{\mathrm{o}}\end{array}$ & M3P & M1V & M2V & M3V & M4V & M5V & M6V & M1P & M2P & M3P & M4P & M5P & M6P \\
\hline 1 & 100.00 & 100.00 & 100.00 & 100.00 & 100.00 & 100.00 & 100.00 & 100.00 & 100.00 & 100.00 & 100.00 & 99.99 & 99.99 \\
\hline 2 & 97.27 & 85.53 & 95.14 & 96.86 & 99.98 & 99.87 & 99.92 & 91.22 & 90.49 & 97.27 & 99.92 & 99.96 & 99.95 \\
\hline 3 & 100.00 & 100.00 & 100.00 & 100.00 & 100.00 & 100.00 & 100.00 & 100.00 & 100.00 & 100.00 & 100.00 & 100.00 & 100.00 \\
\hline 4 & 99.38 & 1.33 & 63.86 & 99.57 & 83.22 & 95.79 & 98.17 & 4.57 & 83.69 & 99.38 & 95.65 & 99.38 & 97.29 \\
\hline 5 & 93.40 & 67.72 & 82.16 & 91.88 & 64.23 & 54.64 & 61.85 & 79.06 & 88.55 & 93.40 & 93.15 & 97.96 & 96.69 \\
\hline 6 & 100.00 & 100.00 & 100.00 & 100.00 & 99.65 & 98.59 & 99.29 & 100.00 & 100.00 & 100.00 & 99.99 & 99.98 & 99.99 \\
\hline 7 & 98.31 & 99.96 & 99.88 & 99.06 & 13.37 & 11.05 & 7.78 & 99.97 & 99.93 & 98.31 & 95.20 & 76.67 & 92.77 \\
\hline 8 & 100.00 & 99.99 & 100.00 & 99.99 & 99.91 & 99.91 & 99.96 & 100.00 & 100.00 & 100.00 & 99.99 & 99.98 & 99.99 \\
\hline 9 & 100.00 & 99.99 & 99.99 & 100.00 & 100.00 & 100.00 & 100.00 & 99.99 & 100.00 & 100.00 & 100.00 & 100.00 & 100.00 \\
\hline 10 & 100.00 & 100.00 & 100.00 & 100.00 & 100.00 & 100.00 & 100.00 & 100.00 & 100.00 & 100.00 & 100.00 & 100.00 & 100.00 \\
\hline 11 & 100.00 & 100.00 & 100.00 & 100.00 & 99.74 & 99.38 & 99.82 & 100.00 & 100.00 & 100.00 & 98.87 & 99.68 & 99.85 \\
\hline 12 & 100.00 & 100.00 & 100.00 & 100.00 & 100.00 & 100.00 & 100.00 & 100.00 & 100.00 & 100.00 & 100.00 & 100.00 & 100.00 \\
\hline 13 & 99.86 & 98.71 & 99.78 & 99.89 & 99.34 & 99.73 & 99.67 & 99.98 & 99.99 & 99.86 & 99.50 & 99.98 & 100.00 \\
\hline 14 & 99.69 & 96.98 & 99.94 & 99.99 & 99.76 & 97.94 & 98.67 & 95.89 & 99.55 & 99.69 & 99.31 & 99.98 & 99.35 \\
\hline 15 & 96.16 & 1.57 & 8.37 & 77.18 & 0.19 & 7.21 & 6.15 & 1.88 & 54.94 & 96.16 & 27.44 & 54.19 & 55.24 \\
\hline 16 & 100.00 & 100.00 & 100.00 & 100.00 & 99.99 & 99.98 & 99.96 & 100.00 & 100.00 & 100.00 & 100.00 & 100.00 & 100.00 \\
\hline 17 & 100.00 & 100.00 & 100.00 & 100.00 & 100.00 & 100.00 & 100.00 & 100.00 & 100.00 & 100.00 & 100.00 & 100.00 & 100.00 \\
\hline 18 & 36.13 & 66.45 & 22.95 & 16.92 & 1.60 & 0.71 & 0.60 & 75.50 & 62.07 & 36.13 & 6.52 & 0.83 & 1.54 \\
\hline 19 & 19.85 & 0.27 & 0.65 & 8.52 & 72.15 & 83.97 & 81.86 & 1.51 & 5.91 & 19.85 & 37.77 & 13.50 & 27.19 \\
\hline 20 & 49.27 & 23.69 & 31.72 & 49.73 & 20.80 & 15.25 & 17.14 & 23.27 & 31.47 & 49.27 & 25.13 & 55.65 & 40.64 \\
\hline 21 & 80.31 & 90.89 & 98.98 & 97.53 & 0.79 & 0.39 & 0.45 & 85.84 & 95.95 & 80.31 & 6.96 & 4.55 & 3.70 \\
\hline 22 & 92.78 & 99.97 & 99.98 & 99.08 & 0.70 & 0.17 & 0.22 & 99.97 & 99.96 & 92.78 & 4.77 & 3.42 & 2.10 \\
\hline 23 & 73.30 & 76.06 & 92.70 & 81.74 & 45.96 & 35.08 & 37.30 & 96.41 & 96.29 & 73.30 & 39.46 & 7.88 & 16.00 \\
\hline 24 & 25.95 & 1.78 & 4.51 & 14.90 & 55.71 & 66.69 & 65.00 & 2.00 & 5.23 & 25.95 & 60.63 & 93.02 & 86.33 \\
\hline 25 & 0.00 & 0.00 & 0.00 & 0.00 & 0.00 & 0.00 & 0.00 & 0.00 & 0.00 & 0.00 & 0.00 & 0.00 & 0.00 \\
\hline 26 & 99.99 & 97.74 & 99.73 & 99.99 & 99.98 & 100.00 & 100.00 & 99.27 & 99.86 & 99.99 & 100.00 & 100.00 & 100.00 \\
\hline 27 & 98.23 & 99.95 & 99.96 & 99.20 & 98.94 & 81.65 & 91.95 & 99.92 & 99.90 & 98.23 & 91.65 & 78.80 & 84.33 \\
\hline 28 & 100.00 & 99.91 & 99.99 & 100.00 & 100.00 & 100.00 & 100.00 & 99.99 & 100.00 & 100.00 & 100.00 & 100.00 & 100.00 \\
\hline 29 & 100.00 & 100.00 & 100.00 & 100.00 & 91.21 & 79.66 & 83.00 & 100.00 & 100.00 & 100.00 & 100.00 & 100.00 & 100.00 \\
\hline 30 & 100.00 & 100.00 & 100.00 & 100.00 & 37.71 & 72.05 & 79.67 & 100.00 & 100.00 & 100.00 & 100.00 & 100.00 & 100.00 \\
\hline 31 & 100.00 & 100.00 & 100.00 & 100.00 & 99.97 & 99.89 & 99.93 & 100.00 & 100.00 & 100.00 & 100.00 & 100.00 & 99.99 \\
\hline 32 & 99.30 & 98.96 & 99.68 & 99.82 & 99.31 & 99.33 & 99.31 & 99.20 & 98.55 & 99.30 & 98.66 & 99.80 & 98.91 \\
\hline 33 & 97.79 & 97.45 & 98.17 & 95.27 & 80.45 & 78.84 & 83.27 & 97.98 & 99.39 & 97.79 & 96.63 & 87.74 & 95.13 \\
\hline 34 & 0.34 & 0.39 & 1.36 & 1.59 & 0.00 & 0.00 & 0.00 & 0.31 & 0.48 & 0.34 & 0.03 & 0.04 & 0.01 \\
\hline 35 & 97.81 & 13.96 & 45.86 & 92.68 & 20.04 & 59.07 & 53.64 & 44.81 & $\begin{array}{l}0.40 \\
85.04\end{array}$ & 97.81 & 99.79 & 98.89 & 99.33 \\
\hline 36 & 74.69 & 79.08 & 71.52 & 68.83 & 45.88 & 97.57 & 98.18 & 94.78 & 86.37 & 74.69 & 54.91 & 10.86 & 27.66 \\
\hline 37 & 99.98 & 99.98 & 99.95 & 99.95 & 99.99 & 99.99 & 99.99 & 100.00 & 99.99 & 99.98 & 100.00 & 100.00 & 100.00 \\
\hline 38 & 99.99 & 99.99 & 99.98 & 99.98 & 99.91 & 99.98 & 99.99 & 100.00 & 100.00 & 99.99 & 99.99 & 99.92 & 99.96 \\
\hline 39 & 100.00 & 100.00 & 100.00 & 100.00 & 100.00 & 100.00 & 100.00 & 100.00 & 100.00 & 100.00 & 100.00 & 100.00 & 100.00 \\
\hline 40 & 77.80 & 0.36 & 4.22 & 22.19 & 2.24 & 50.36 & 41.86 & 0.76 & 23.55 & 77.80 & 1.46 & 48.00 & 64.73 \\
\hline 41 & 100.00 & 100.00 & 100.00 & 100.00 & 100.00 & 100.00 & 100.00 & 100.00 & 100.00 & 100.00 & 100.00 & 100.00 & 100.00 \\
\hline 42 & 100.00 & 99.99 & 99.98 & 99.99 & 99.62 & 99.93 & 99.95 & 100.00 & 100.00 & 100.00 & 99.99 & 99.98 & 99.97 \\
\hline 43 & 100.00 & 100.00 & 100.00 & 100.00 & 99.91 & 99.87 & 99.80 & 100.00 & 100.00 & 100.00 & 100.00 & 100.00 & 100.00 \\
\hline 44 & 99.96 & 99.80 & 99.82 & 99.92 & 100.00 & 100.00 & 100.00 & 99.98 & 99.95 & 99.96 & 100.00 & 100.00 & 100.00 \\
\hline 45 & 86.39 & 99.30 & 99.45 & 99.04 & 0.02 & 0.01 & 0.01 & 96.35 & 98.44 & 86.39 & 3.08 & 23.20 & 3.54 \\
\hline 46 & 99.82 & 99.99 & 99.99 & 99.92 & $\begin{array}{l}0.02 \\
7.98\end{array}$ & $\begin{array}{l}0.11 \\
1.39\end{array}$ & $\begin{array}{l}0.61 \\
1.61\end{array}$ & 99.99 & 99.99 & 99.82 & 95.32 & 96.15 & 94.25 \\
\hline $\begin{array}{l}40 \\
47\end{array}$ & 100.00 & 100.00 & 100.00 & 100.00 & 100.00 & 100.00 & 100.00 & 100.00 & 100.00 & 100.00 & 100.00 & 100.00 & 100.00 \\
\hline 48 & 98.66 & 63.42 & 90.45 & 92.61 & 97.61 & 98.81 & 99.33 & 69.30 & 93.08 & 98.66 & 99.31 & 98.83 & 99.42 \\
\hline
\end{tabular}


Table S5. Ranges employed for the determination of the dependence of DP4+ with $[\mu, \sigma, v]$ (Figure $3 b$ ), and for the random generation of $[\mu, \sigma, v]$ shown in Figure 4a.

\begin{tabular}{|c|c|c|c|}
\hline Parameter & $\operatorname{Min}\left(x_{m}\right)$ & $\operatorname{Max}\left(\mathbf{x}_{M}\right)$ & $\Delta$ \\
\hline${ }^{13} \mathrm{C} \mu_{\mathrm{u}, \mathrm{sp} 2}$ & -15.0 & 9.0 & 24.0 \\
\hline${ }^{13} \mathrm{C} \mu \mu_{\mathrm{u}, \mathrm{sp} 3}$ & -8.0 & 0.0 & 8.0 \\
\hline${ }^{1} \mathrm{H} \mu \mathrm{u,sp2}$ & -1.0 & 1.5 & 2.5 \\
\hline${ }^{1} \mathrm{H} \mu \mathrm{u}, \mathrm{sp} 3$ & -0.5 & 1.0 & 1.5 \\
\hline${ }^{13} \mathrm{C} \sigma_{\mathrm{u}, \mathrm{sp} 2}$ & 1.7 & 3.8 & 2.1 \\
\hline${ }^{13} \mathrm{C} \sigma_{\mathrm{u}, \mathrm{sp} 3}$ & 1.2 & 4.1 & 2.9 \\
\hline${ }^{1} \mathrm{H} \sigma_{\mathrm{u}, \mathrm{sp} 2}$ & 0.1 & 0.5 & 0.4 \\
\hline${ }^{1} \mathrm{H} \sigma_{\mathrm{u}, \mathrm{sp} 3}$ & 0.1 & 0.4 & 0.3 \\
\hline${ }^{13} \mathrm{C} \sigma_{\mathrm{s}}$ & 1.2 & 4.5 & 3.3 \\
\hline${ }^{1} \mathrm{H} \sigma_{\mathrm{s}}$ & 0.1 & 0.4 & 0.3 \\
\hline${ }^{13} \mathrm{C} v_{\mathrm{u}, \mathrm{sp} 2}$ & 3.0 & 15.0 & 12.0 \\
\hline${ }^{13} \mathrm{C} \mathrm{v}{ }_{\mathrm{u}, \mathrm{sp} 3}$ & 3.0 & 15.0 & 12.0 \\
\hline${ }^{1} \mathrm{H} v_{\mathrm{u}, \mathrm{sp} 2}$ & 3.0 & 15.0 & 12.0 \\
\hline${ }^{1} \mathrm{H} v_{\mathrm{u}, \mathrm{sp} 3}$ & 3.0 & 15.0 & 12.0 \\
\hline${ }^{13} \mathrm{C} v_{\mathrm{s}}$ & 3.0 & 15.0 & 12.0 \\
\hline${ }^{1} \mathrm{H} v_{\mathrm{s}}$ & 3.0 & 15.0 & 12.0 \\
\hline
\end{tabular}

Table S6. Ranges employed for the random generation of $[\mu, \sigma, v]$ shown in Figure $4 \mathrm{~b}$.

\begin{tabular}{|c|c|c|c|}
\hline Parameter & Min & Max & $\Delta$ \\
\hline${ }^{13} \mathrm{C} \mu_{\mathrm{u}, \mathrm{sp} 2}$ & 0.620 & 1.220 & 0.600 \\
\hline${ }^{13} \mathrm{C} \mu_{\mathrm{u}, \mathrm{sp} 3}$ & -3.109 & -2.709 & 0.400 \\
\hline${ }^{1} \mathrm{H} \mu_{\mathrm{u}, \mathrm{sp} 2}$ & -0.367 & -0.327 & 0.040 \\
\hline${ }^{1} \mathrm{H} \mu_{\mathrm{u}, \mathrm{s} \mathbf{3} 3}$ & 0.008 & 0.028 & 0.020 \\
\hline${ }^{13} \mathrm{C} \sigma_{\mathrm{u}, \mathrm{sp} 2}$ & 1.348 & 2.148 & 0.800 \\
\hline${ }^{13} \mathrm{C} \sigma_{\mathrm{u}, \mathrm{sp} \mathrm{p}}$ & 1.400 & 1.800 & 0.400 \\
\hline${ }^{1} \mathrm{H} \sigma_{\mathrm{u}, \mathrm{sp} 2}$ & 0.098 & 0.138 & 0.040 \\
\hline${ }^{1} \mathrm{H} \sigma_{\mathrm{u}, \mathrm{pp} 3}$ & 0.092 & 0.132 & 0.040 \\
\hline${ }^{13} \mathrm{C} \sigma_{\mathrm{s}}$ & 1.357 & 1.757 & 0.400 \\
\hline${ }^{1} \mathrm{H} \sigma_{\mathrm{s}}$ & 0.094 & 0.114 & 0.020 \\
\hline${ }^{13} \mathrm{C} v_{\mathrm{u}, \mathrm{sp} 2}$ & 4.000 & 6.000 & 2.000 \\
\hline${ }^{13} \mathrm{C} v_{\mathrm{u}, \mathrm{sp} 3}$ & 5.000 & 7.000 & 2.000 \\
\hline${ }^{1} \mathrm{H} v_{\mathrm{u}, \mathrm{sp} 2}$ & 3.000 & 5.000 & 2.000 \\
\hline${ }^{1} \mathrm{H} v_{\mathrm{u}, \mathrm{p} 3}$ & 2.000 & 4.000 & 2.000 \\
\hline${ }^{13} \mathrm{C} v_{\mathrm{s}}$ & 5.000 & 7.000 & 2.000 \\
\hline${ }^{1} \mathrm{H} v_{\mathrm{s}}$ & 2.000 & 4.000 & 2.000 \\
\hline
\end{tabular}


Table S7. Dependence of DP4+ with each of its $16[\mu, \sigma, v]$ parameters determined by correlating the GIAO NMR chemical shifts computed at the $\mathrm{PCM} / \mathrm{mPW} 1 \mathrm{PW} 91 / 6-31+\mathrm{G}^{* *} / \mathrm{B} 3 \mathrm{LYP} / 6-31 \mathrm{G}^{*}$ level of theory (M3P), with $15[\mu, \sigma, v]_{0}$ parameters estimated at the M3P level and allowing the remaining one to oscillate between 6 levels. The results obtained with the matched M3P parameters are shown in grey.

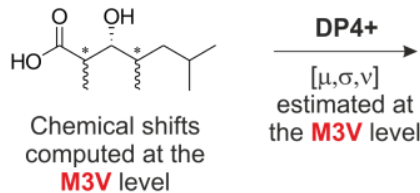

Matched

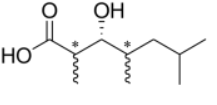

Chemical shifts
computed at the M3V level

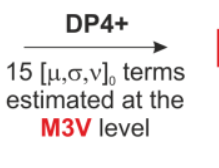

The remaining term oscillating in 6 levels

\begin{tabular}{|c|c|c|c|c|c|c|c|c|c|c|c|c|c|c|c|c|c|c|c|}
\hline \multirow[b]{2}{*}{ Comp $\mathrm{N}^{\circ}$} & \multirow[b]{2}{*}{ M3P } & \multicolumn{6}{|c|}{${ }^{13} \mathrm{C} \mu_{\mathrm{u}, \mathrm{sp} 2}$} & \multicolumn{6}{|c|}{${ }^{13} \mathrm{C} \mu_{\mathrm{u}, \mathrm{sp} 3}$} & \multicolumn{6}{|c|}{${ }^{1} \mathbf{H} \mu_{\mathrm{u}, \mathrm{sp} 2}$} \\
\hline & & $\mathbf{x}_{\mathrm{m}}$ & $\mathbf{x}_{\mathrm{m}}+\mathbf{1} / \mathbf{5} \Delta$ & $x_{m}+2 / 5 \Delta$ & $\mathbf{x}_{\mathrm{m}}+\mathbf{3} / \mathbf{5 \Delta}$ & $x_{\mathrm{m}}+4 / 5 \Delta$ & $\mathbf{x}_{\mathrm{M}}$ & $\mathbf{x}_{\mathrm{m}}$ & $x_{m}+1 / 5 \Delta$ & $x_{m}+2 / 5 \Delta$ & $\mathbf{x}_{\mathrm{m}}+3 / 5 \Delta$ & $\mathbf{x}_{\mathrm{m}}+\mathbf{4} / \mathbf{5 \Delta}$ & $\mathbf{x}_{\mathbf{M}}$ & $\mathbf{x}_{\mathrm{m}}$ & $x_{m}+1 / 5 \Delta$ & $x_{m}+2 / 5 \Delta$ & $x_{m}+3 / 5 \Delta$ & $x_{m}+4 / 5 \Delta$ & $\mathbf{x}_{\mathrm{M}}$ \\
\hline 1 & 100.00 & 100.00 & 100.00 & 100.00 & 100.00 & 100.00 & 100.00 & 99.75 & 99.97 & 100.00 & 100.00 & 100.00 & 100.00 & 100.00 & 100.00 & 100.00 & 100.00 & 100.00 & 100.00 \\
\hline 2 & 97.27 & 99.45 & 99.49 & 99.61 & 99.70 & 98.39 & 99.03 & 99.35 & 99.60 & 99.62 & 97.92 & 90.76 & 16.00 & 67.71 & 97.60 & 96.04 & 93.92 & $\begin{array}{l}92.69 \\
\end{array}$ & 91.93 \\
\hline 3 & 100.00 & 100.00 & 100.00 & 100.00 & 100.00 & 100.00 & 100.00 & 95.63 & 99.41 & 99.97 & 100.00 & 100.00 & 100.00 & 100.00 & 100.00 & 100.00 & 100.00 & 100.00 & 100.00 \\
\hline 4 & 99.38 & 95.60 & 94.49 & 92.09 & 97.80 & 99.31 & 98.89 & 99.80 & 99.92 & 99.94 & 99.58 & 66.03 & 0.07 & 99.92 & 99.41 & 99.68 & 99.74 & 99.76 & 99.77 \\
\hline 5 & 93.40 & 93.48 & 93.47 & 93.44 & 93.40 & 93.64 & 93.66 & 95.91 & 96.53 & 96.75 & 94.29 & 87.57 & 67.82 & 93.40 & 93.40 & 93.40 & 93.40 & 93.40 & 93.40 \\
\hline 6 & 100.00 & 100.00 & 100.00 & 100.00 & 100.00 & 99.99 & 99.99 & 99.58 & 99.71 & 99.95 & 100.00 & 100.00 & 100.00 & 100.00 & 100.00 & 100.00 & 100.00 & 100.00 & 100.00 \\
\hline 7 & 98.31 & 97.04 & 97.37 & 97.88 & 98.41 & 90.12 & 90.15 & 68.19 & 61.03 & 74.60 & 97.19 & 99.93 & 99.99 & 98.31 & 98.31 & 98.31 & 98.31 & 98.31 & 98.31 \\
\hline 8 & 100.00 & 100.00 & 100.00 & 100.00 & 100.00 & 100.00 & 100.00 & 99.57 & 99.63 & 99.90 & 100.00 & 99.98 & 99.84 & 100.00 & 100.00 & 100.00 & 100.00 & 100.00 & 100.00 \\
\hline 9 & 100.00 & 100.00 & 100.00 & 100.00 & 100.00 & 100.00 & 100.00 & 99.99 & 100.00 & 100.00 & 100.00 & 99.99 & 99.83 & 100.00 & 100.00 & 100.00 & 100.00 & 100.00 & 100.00 \\
\hline 10 & 100.00 & 100.00 & 100.00 & 100.00 & 100.00 & 100.00 & 100.00 & 100.00 & 100.00 & 100.00 & 100.00 & 100.00 & 100.00 & 100.00 & 100.00 & 100.00 & 100.00 & 100.00 & 100.00 \\
\hline 11 & 100.00 & $\begin{array}{l}99.99 \\
\end{array}$ & 99.96 & 99.98 & 100.00 & 100.00 & 100.00 & 99.15 & 99.98 & 100.00 & 100.00 & 100.00 & 100.00 & 100.00 & 100.00 & 100.00 & 100.00 & 100.00 & 100.00 \\
\hline 12 & 100.00 & 100.00 & 100.00 & 100.00 & 100.00 & 100.00 & 100.00 & 100.00 & 100.00 & 100.00 & 100.00 & 100.00 & 100.00 & 100.00 & 100.00 & 100.00 & 100.00 & 100.00 & 100.00 \\
\hline 13 & 99.86 & 99.91 & 99.93 & 99.96 & 99.93 & 99.99 & 95.47 & 90.26 & 99.91 & 99.98 & 99.89 & 99.67 & 99.61 & 99.71 & 99.89 & 99.91 & 99.89 & 99.87 & 99.87 \\
\hline 14 & 99.69 & 99.18 & 98.78 & 97.72 & 99.56 & 99.97 & 99.96 & 99.91 & 99.93 & 99.97 & 99.77 & 90.02 & 1.85 & 95.93 & 99.72 & 99.75 & 99.58 & 99.47 & 99.40 \\
\hline 15 & 96.16 & 42.18 & 36.16 & 27.65 & 76.49 & 79.42 & 66.52 & 99.54 & 99.61 & 99.40 & 97.25 & 72.18 & 10.97 & 99.57 & 96.09 & 96.73 & 97.71 & 98.04 & 98.21 \\
\hline 16 & 100.00 & 100.00 & 100.00 & 100.00 & $\begin{array}{l}100.09 \\
100\end{array}$ & 100.00 & 100.00 & 100.00 & 100.00 & 100.00 & 100.00 & 100.00 & 100.00 & 99.99 & 100.00 & 100.00 & 100.00 & $\begin{array}{l}\$ 0.04 \\
100.00\end{array}$ & $\begin{array}{l}90.21 \\
100.00\end{array}$ \\
\hline 17 & 100.00 & 100.00 & 100.00 & 100.00 & 100.00 & 100.00 & 100.00 & 100.00 & 100.00 & 100.00 & 100.00 & 100.00 & 100.00 & 100.00 & 100.00 & 100.00 & 100.00 & 100.00 & 100.00 \\
\hline 18 & 36.13 & 31.83 & 30.57 & 28.03 & 21.95 & 38.66 & 43.55 & 0.59 & 0.42 & 0.80 & 21.87 & 73.08 & 95.32 & 36.13 & 36.13 & 36.13 & 36.13 & 36.13 & 36.13 \\
\hline 19 & 19.85 & 24.67 & 25.53 & 26.96 & 23.09 & 19.80 & 19.29 & 26.22 & 23.95 & 14.31 & 16.46 & 14.17 & 0.82 & 19.85 & 19.85 & 19.85 & 19.85 & 19.85 & 19.85 \\
\hline 20 & 49.27 & 42.69 & 41.60 & 39.84 & 42.87 & 49.50 & 50.42 & 35.03 & 38.75 & 61.44 & 54.48 & 27.13 & 12.50 & 49.27 & 49.27 & 49.27 & 49.27 & 49.27 & 49.27 \\
\hline 21 & 80.31 & 47.90 & 43.64 & 37.13 & 66.38 & 79.27 & 68.31 & 19.08 & 13.60 & 8.75 & 71.73 & 89.43 & 71.83 & 80.31 & 80.31 & 80.31 & 80.31 & 80.31 & 80.31 \\
\hline 22 & 92.78 & 63.16 & 58.31 & 50.37 & 82.16 & 87.65 & 82.95 & 0.98 & 0.78 & 4.46 & 87.85 & 99.90 & 99.79 & 92.78 & 92.78 & 92.78 & 92.78 & 92.78 & 92.78 \\
\hline 23 & 73.30 & 73.65 & 73.99 & 73.64 & 61.15 & 74.75 & 74.23 & 3.58 & 3.33 & 5.77 & 54.90 & 97.14 & 62.98 & 73.30 & 73.30 & 73.30 & 73.30 & 73.30 & 73.30 \\
\hline 24 & 25.95 & 25.66 & 25.40 & 25.61 & 37.42 & 24.66 & 25.11 & 96.30 & 96.55 & 93.69 & 39.16 & 4.95 & 1.56 & 25.95 & 25.95 & 25.95 & 25.95 & 25.95 & 25.95 \\
\hline 25 & 0.00 & 0.00 & 0.00 & 0.00 & 0.00 & $\begin{array}{c}2.00 \\
0.00\end{array}$ & 0.00 & 0.00 & 0.00 & 0.00 & 0.00 & 0.0 & 0.0 & 0.00 & 0.00 & 0.00 & 0.00 & 0.00 & 0.00 \\
\hline 26 & 99.99 & 99.99 & 99.99 & 99.99 & 99.99 & 99.99 & 99.99 & 100.00 & 100.00 & 100.00 & 100.00 & 99.58 & 55. & 99.99 & $\begin{array}{l}0.00 \\
99.99\end{array}$ & $\begin{array}{l}0.00 \\
99.99\end{array}$ & $\begin{array}{l}0.00 \\
99.99\end{array}$ & $\begin{array}{l}0.00 \\
99.99\end{array}$ & $\begin{array}{l}0.00 \\
99.99\end{array}$ \\
\hline 27 & 98.23 & 98.23 & 98.23 & 98.23 & 98.23 & 98.23 & 98.23 & 0.04 & 1.27 & 49.14 & 96.94 & 99.93 & 99.98 & 98.23 & 98.23 & 98.23 & 98.23 & 98.23 & 98.23 \\
\hline 28 & 100.00 & 100.00 & 100.00 & 100.00 & 100.00 & 100.00 & 100.00 & 100.00 & 100.00 & 100.00 & 100.00 & 99.99 & 98.79 & 99.98 & 100.00 & 100.00 & 100.00 & 100.00 & 100.00 \\
\hline 29 & 100.00 & 100.00 & 100.00 & 100.00 & 100.00 & 100.00 & 100.00 & 99.16 & 99.97 & 100.00 & 100.00 & 100.00 & 100.00 & 100.00 & 100.00 & 100.00 & 100.00 & 100.00 & 100.00 \\
\hline 30 & 100.00 & 100.00 & 100.00 & 100.00 & 100.00 & 100.00 & 100.00 & 100.00 & 100.00 & 100.00 & 100.00 & 100.00 & 99.96 & 100.00 & 100.00 & 100.00 & 100.00 & 100.00 & 100.00 \\
\hline 31 & 100.00 & 100.00 & 100.00 & 100.00 & 100.00 & 100.00 & 100.00 & 97.47 & 99.87 & 99.98 & 100.00 & 100.00 & 100.00 & 100.00 & 100.00 & 100.00 & 100.00 & 100.00 & 100.00 \\
\hline 32 & 99.30 & 99.67 & 99.61 & 99.49 & 99.22 & 99.94 & 99.94 & 69.24 & 82.80 & 99.25 & 99.47 & 93.35 & 28.94 & 93.47 & 99.29 & 98.83 & 98.42 & 98.15 & 97.99 \\
\hline 33 & 97.79 & 96.48 & 97.01 & 97.76 & 98.36 & & 83.70 & 32. & 41. & 81.44 & 97.04 & 99.64 & 99 . & 99.8 & 97.73 & 98.7 & 99.09 & 99.23 & 99.29 \\
\hline 34 & 0.34 & 0.41 & 0.33 & 0.23 & 0.12 & 2.80 & 1.91 & 0.00 & 0.01 & 0.04 & 0.22 & 0.09 & 0.00 & 3.34 & 0.34 & 10.12 & 9.51 & 8.99 & 8.91 \\
\hline 35 & 97.81 & $\begin{array}{l}0.41 \\
96.61\end{array}$ & 96.80 & 97.06 & 96.84 & 94.11 & 93.84 & 99.87 & 99.88 & 99.46 & 98.43 & $\begin{array}{l}0.09 \\
77.08\end{array}$ & 12.79 & 95.97 & 97.80 & 99.46 & 99.21 & 99.07 & $\begin{array}{l}0.91 \\
98.97\end{array}$ \\
\hline 36 & 74.69 & 61.07 & 59.09 & 55.80 & 68.37 & 74.74 & 72.11 & 54.75 & 54.19 & 59.31 & 71.02 & 87. & 90. & 99. & 72.57 & 90. & 95.80 & 97.19 & 97.77 \\
\hline 37 & 99.98 & 99.99 & & 99.99 & & 99. & 99 & 99 & 99. & 99. & 99. & 99 & 99 . & 99.98 & 99 . & 99 . & 99.93 & 99.91 & 99.90 \\
\hline 38 & 99.99 & 99.98 & 99.98 & 99.97 & 99.99 & 99.99 & 99.99 & 99.99 & 99.99 & 99.99 & 99.99 & 99.99 & 99.99 & 100.00 & 99.99 & 99.99 & 100.00 & 100.00 & 100.00 \\
\hline 39 & 100.00 & 100.00 & 100.00 & 100.00 & 100.00 & 100.00 & 100.00 & 100.00 & 100.00 & 100.00 & 100.00 & 100.00 & 100.00 & 100.00 & 100.00 & 100.00 & 100.00 & 100.00 & 100.00 \\
\hline 40 & 77.80 & 62.86 & 60.33 & 55.72 & 52.35 & 82.30 & 79.13 & 89.97 & 89.70 & 89.61 & 73.75 & 79.75 & 80.20 & & 75.42 & 1.28 & 7.67 & 15.97 & 22.42 \\
\hline 41 & 100.00 & 100.00 & 100.00 & 100.00 & 100.00 & 100.00 & 100.00 & 100.00 & 100.00 & 100.00 & 100.00 & 100.00 & 100.00 & 100.00 & 100.00 & 100.00 & 100.00 & 100.00 & 100.00 \\
\hline 42 & 100.00 & 99.99 & 99.99 & 99.98 & & 100 & 100.00 & 100 & 100.00 & 100.00 & 100.00 & 100.00 & 100.00 & 100.00 & 100.00 & 100.00 & 100.00 & 100.00 & 100.00 \\
\hline 43 & 100.00 & 100.00 & 100.00 & 100.00 & 100.00 & 100.00 & 100.00 & 100.00 & 100.00 & 100.00 & 100.00 & 100.00 & 100.00 & 100.00 & 100.00 & 100.00 & 100.00 & 100.00 & 100.00 \\
\hline 44 & 99.96 & 99.99 & 100.00 & 100.00 & 99.99 & 99.95 & 99.97 & 100.00 & 100.00 & 100.00 & 99.97 & 99.85 & 99.59 & 99.96 & 99.96 & 99.96 & 99.96 & 99.96 & 99.96 \\
\hline 45 & 86.39 & 52.48 & 46.26 & 36.08 & 53.89 & 90.02 & 85.40 & 34.91 & 30.30 & 38.56 & 83. & 95. & 98. & 86. & 86. & 86.39 & 86.39 & 86.39 & 86.39 \\
\hline 46 & 99.82 & 99.82 & 99.82 & 99.82 & & 99. & & 3.1 & 20. & 84. & 99.66 & 99.98 & 99. & 99.82 & 99.8 & 99.82 & 99.82 & 99.82 & 99.82 \\
\hline 47 & 100.00 & 100.00 & 100.00 & 100.00 & 100.00 & 100.00 & 100.00 & 99.95 & 99.99 & 100.00 & 100.00 & 100.00 & 100.00 & 100.00 & 100.00 & 100.00 & 100.00 & 100.00 & 100.00 \\
\hline 48 & 98.66 & 98.94 & 98.97 & 99.02 & 98.68 & 98.69 & 98.74 & 95.34 & 97.45 & 98.28 & 98.67 & 97.13 & 82.15 & 98.66 & 98.66 & 98.66 & 98.66 & 98.66 & 98.66 \\
\hline
\end{tabular}


Table S7 (cont). Dependence of DP4+ with each of its $16[\mu, \sigma, v]$ parameters determined by correlating the GIAO NMR chemical shifts computed at the $\mathrm{PCM} / \mathrm{mPW} 1 \mathrm{PW} 91 / 6-31+\mathrm{G}^{* *} / / \mathrm{B} 3 \mathrm{LYP} / 6-31 \mathrm{G}^{*}$ level of theory (M3P), with $15[\mu, \sigma, v]_{0}$ parameters estimated at the M3P level and allowing the remaining one to oscillate between 6 levels. The results obtained with the matched M3P parameters are shown in grey.

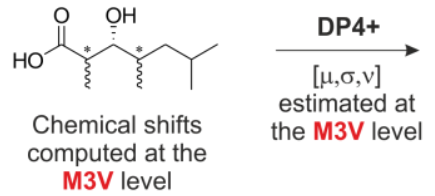

Matched

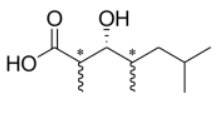

Chemical shifts
computed at the M3V level

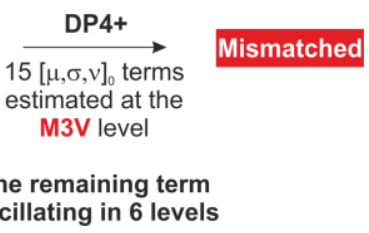

oscillating in 6 levels

\begin{tabular}{|c|c|c|c|c|c|c|c|c|c|c|c|c|c|c|c|c|c|c|c|}
\hline \multirow[b]{2}{*}{ Comp $\mathrm{N}^{\circ}$} & \multirow[b]{2}{*}{ M3P } & \multicolumn{6}{|c|}{${ }^{1} \mathbf{H} \mu_{\mathrm{u}, \mathrm{sp} 3}$} & \multicolumn{6}{|c|}{${ }^{13} \mathrm{C} \sigma_{\mathrm{u}, \mathrm{sp} 2}$} & \multicolumn{6}{|c|}{${ }^{13} \mathrm{C} \sigma_{\mathrm{u}, \mathrm{sp} 3}$} \\
\hline & & $\mathbf{x}_{\mathrm{m}}$ & $x_{m}+1 / 5 \Delta$ & $x_{m}+2 / 5 \Delta$ & $\mathbf{x}_{\mathrm{m}}+3 / 5 \Delta$ & $x_{\mathrm{m}}+4 / 5 \Delta$ & $\mathbf{x}_{\mathbf{M}}$ & $\mathbf{x}_{\mathrm{m}}$ & $x_{m}+1 / 5 \Delta$ & $x_{m}+2 / 5 \Delta$ & $\mathbf{x}_{\mathrm{m}}+3 / 5 \Delta$ & $x_{m}+4 / 5 \Delta$ & $\mathbf{x}_{\mathbf{M}}$ & $\mathbf{x}_{\mathrm{m}}$ & $x_{m}+1 / 5 \Delta$ & $x_{m}+2 / 5 \Delta$ & $x_{\mathrm{m}}+3 / 5 \Delta$ & $x_{m}+4 / 5 \Delta$ & $\mathbf{x}_{\mathbf{M}}$ \\
\hline 1 & 100.00 & 100.00 & 100.00 & 100.00 & 100.00 & 100.00 & 100.00 & 100.00 & 100.00 & 100.00 & 100.00 & 100.00 & 100.00 & 100.00 & 100.00 & 100.00 & 100.00 & 100.00 & 100.00 \\
\hline 2 & 97.27 & 99.70 & 98.13 & 98.57 & 98.69 & 98.74 & 98.76 & 97.12 & 98.01 & 98.41 & 98.63 & 98.76 & 98.85 & 99.26 & 95.70 & 87.93 & 78.15 & 68.99 & 61.49 \\
\hline 3 & 100.00 & 100.00 & 100.00 & 100.00 & 100.00 & 100.00 & 100.00 & 100.00 & 100.00 & 100.00 & 100.00 & 100.00 & 100.00 & 100.00 & 100.00 & 100.00 & 100.00 & 100.00 & 100.00 \\
\hline 4 & 99.38 & 80.88 & 99.70 & 99.88 & 99.72 & 99.55 & 99.42 & 99.40 & 99.18 & 98.99 & 98.83 & 98.69 & 98.58 & 99.96 & 98.45 & 88.98 & 69.90 & 50.73 & 36.95 \\
\hline 5 & 93.40 & 32.77 & 95.16 & 51.98 & 51.21 & 59.99 & 64.43 & 93.39 & 93.43 & 93.45 & 93.46 & 93.47 & 93.48 & 95.66 & 92.36 & 89.26 & 86.76 & 84.84 & 83.36 \\
\hline 6 & 100.00 & 99.98 & 100.00 & 99.99 & 99.33 & 99.46 & 99.80 & 100.00 & 100.00 & 100.00 & 100.00 & 100.00 & 100.00 & 100.00 & 100.00 & 99.99 & 99.99 & 99.99 & 99.98 \\
\hline 7 & 98.31 & 81.50 & 97.99 & 96.34 & 94.29 & 93.07 & 92.15 & 98.37 & 97.94 & 97.61 & 97.35 & 97.15 & 96.99 & 96.41 & 98.70 & 99.30 & 99.53 & 99.63 & 99.69 \\
\hline 8 & $\begin{array}{l}300.00 \\
100.00\end{array}$ & 100.00 & 100.00 & 99.95 & 99.95 & 99.97 & 99.98 & 100.00 & 100.00 & 100.00 & 100.00 & 100.00 & 100.00 & $\begin{array}{l}30.41 \\
100.00\end{array}$ & 99.99 & 99.98 & 99.97 & 99.95 & 99.93 \\
\hline 9 & 100.00 & 100.00 & 100.00 & 100.00 & 100.00 & 100.00 & 100.00 & 100.00 & 100.00 & 100.00 & 100.00 & 100.00 & 100.00 & 100.00 & 100.00 & 100.00 & 100.00 & 100.00 & 100.00 \\
\hline 10 & 100.00 & 100.00 & 100.00 & 100.00 & 100.00 & 100.00 & 100.00 & 100.00 & 100.00 & 100.00 & 100.00 & 100.00 & 100.00 & 100.00 & 100.00 & 100.00 & 100.00 & 100.00 & 100.00 \\
\hline 11 & 100.00 & 100.00 & 100.00 & 100.00 & 100.00 & 100.00 & 100.00 & 100.00 & 100.00 & 100.00 & 100.00 & 100.00 & 100.00 & 100.00 & 100.00 & 100.00 & 100.00 & 100.00 & 100.00 \\
\hline 12 & 100.00 & 100.00 & 100.00 & 100.00 & 100.00 & 100.00 & 100.00 & 100.00 & 100.00 & 100.00 & 100.00 & 100.00 & 100.00 & 100.00 & 100.00 & 100.00 & 100.00 & 100.00 & 100.00 \\
\hline 13 & 99.86 & 98.48 & 99.57 & 14.57 & 27.08 & 37.42 & 43.03 & 99.85 & 99.87 & 99.87 & 99.86 & 99.85 & 99.84 & 99.79 & 99.87 & 99.90 & 99.92 & 99.92 & 99.93 \\
\hline 14 & 99.69 & 98.11 & 99.97 & 99.99 & 99.25 & 95.54 & 99.13 & 99.69 & 99.71 & 99.71 & 99.71 & 99.72 & 99.72 & 99.93 & 99.48 & 98.36 & 96.72 & 94.89 & 93.10 \\
\hline 15 & 96.16 & 5.56 & 94.29 & 99.87 & 99.86 & 99.34 & 98.46 & 96.50 & 93.19 & 89.60 & 86.20 & 83.16 & 80.54 & 98.01 & 95.32 & 92.92 & 91.07 & 89.66 & 88.57 \\
\hline 16 & 100.00 & 99.99 & 100.00 & 99.99 & 99.99 & 99.99 & 99.99 & 100.00 & 100.00 & 100.00 & 100.00 & 100.00 & 100.00 & 100.00 & 100.00 & 100.00 & 100.00 & 100.00 & 100.00 \\
\hline 17 & 100.00 & 100.00 & 100.00 & 99.99 & 99.99 & 99.99 & 99.99 & 100.00 & 100.00 & 100.00 & 100.00 & 100.00 & 100.00 & 100.00 & 100.00 & 100.00 & 100.00 & 100.00 & 100.00 \\
\hline 18 & 36.13 & 51.75 & 13.23 & 1.88 & 4.51 & 6.89 & 8.62 & 36.18 & 35.90 & 35.82 & 35.80 & 35.82 & 35.83 & 21.41 & 40.93 & 50.65 & 55.54 & 58.31 & 60.03 \\
\hline 19 & 19.85 & 81.57 & 9.73 & 11.36 & 25.42 & 27.88 & 29.63 & 19.75 & 20.43 & 20.86 & 21.15 & 21.35 & 21.51 & 16.28 & 20.81 & 22.45 & 22.96 & 23.02 & 22.91 \\
\hline 20 & 49.27 & 17.25 & 54.87 & 79.81 & 70.40 & 64.56 & 61.04 & 49.41 & 48.43 & 47.83 & 47.43 & 47.15 & 46.94 & 55.90 & 47.24 & 42.92 & 40.47 & 38.92 & 37.86 \\
\hline 21 & 80.31 & 12.04 & 90.51 & 89.62 & 85.17 & 82.20 & 80.08 & 80.84 & 76.77 & 73.68 & 71.32 & 69.51 & 68.08 & 71.80 & 82.38 & 86.12 & 87.90 & 88.90 & 89.54 \\
\hline 22 & 92.78 & 72.84 & 97.47 & $\begin{array}{l}03.02 \\
93.18\end{array}$ & 92.44 & 91.63 & $\begin{array}{l}0.00 \\
91.09\end{array}$ & $\begin{array}{l}0.04 \\
93.12\end{array}$ & 90.38 & 88.08 & 86.21 & 84.71 & $\begin{array}{l}80.00 \\
83.49\end{array}$ & 89.11 & 93.54 & 94.79 & 95.34 & $\begin{array}{l}0.90 \\
95.63\end{array}$ & 95.81 \\
\hline 23 & 73.30 & 96.62 & 53.01 & 51.60 & 65.16 & 70.15 & 72.93 & 73.38 & 72.93 & 72.82 & 72.83 & 72.88 & 72.94 & 77.89 & 71.84 & 68.65 & 66.76 & 65.52 & 64.65 \\
\hline 24 & 25.95 & 3.78 & 36.41 & 49.57 & 37.02 & 30.60 & 27.25 & 25.88 & 26.23 & 26.29 & 26.27 & 26.22 & 26.17 & 20.28 & 27.61 & 31.04 & 32.99 & 34.24 & 35.13 \\
\hline 25 & 0.00 & 0.00 & 0.00 & 0.00 & 0.00 & 0.00 & 0.00 & 0.00 & 0.00 & 0.00 & 0.00 & 0.00 & 0.00 & 0.00 & 0.00 & 0.00 & 0.00 & 0.00 & 0.00 \\
\hline 26 & 99.99 & 99.90 & 100.00 & 100.00 & 100.00 & 99.99 & 99.98 & 99.99 & 99.99 & 99.99 & 99.99 & 99.99 & 99.99 & 100.00 & 99.99 & 99.92 & 99.73 & 99.41 & 98.97 \\
\hline $\begin{array}{l}20 \\
27\end{array}$ & 98.23 & 99.90 & 98.16 & $\begin{array}{r}99.73 \\
\end{array}$ & 99.92 & 99.96 & 99.97 & 98.23 & 98.23 & 98.23 & 98.23 & 98.23 & 98.23 & 99.53 & 97.11 & 90.60 & 80.44 & 69.31 & 59.37 \\
\hline 28 & 100.00 & 100.00 & 100.00 & 100.00 & 100.00 & 100.00 & 100.00 & 100.00 & 100.00 & 100.00 & 100.00 & 100.00 & 100.00 & 100.00 & 100.00 & 99.99 & 99.97 & 99.95 & 99.92 \\
\hline 29 & 100.00 & 99.87 & 100.00 & 100.00 & 100.00 & 100.00 & 100.00 & 100.00 & 100.00 & 100.00 & 100.00 & 100.00 & 100.00 & 100.00 & 100.00 & 100.00 & 100.00 & 100.00 & 100.00 \\
\hline 30 & 100.00 & 8.37 & 100.00 & 100.00 & 100.00 & 100.00 & 99.99 & 100.00 & 100.00 & 100.00 & 100.00 & 100.00 & 100.00 & 100.00 & 100.00 & 100.00 & 100.00 & 100.00 & 100.00 \\
\hline 31 & 100.00 & 100.00 & 100.00 & 98.65 & 86.81 & 96.96 & 98.69 & 100.00 & 100.00 & 100.00 & 100.00 & 100.00 & 100.00 & 100.00 & 100.00 & 100.00 & 100.00 & 100.00 & 100.00 \\
\hline 32 & 99.30 & 98.21 & 99.83 & 99.97 & 99.95 & 99.92 & 99.90 & 99.27 & 99.46 & 99.56 & 99.61 & 99.65 & 99.68 & 99.81 & 98.93 & 97.34 & 95.46 & 93.64 & 92.02 \\
\hline 33 & 97.79 & 97.83 & 96.82 & 43.29 & 61.79 & 71.82 & 76.55 & 97.86 & 97.31 & 96.86 & 96.51 & 96.23 & 96.01 & 98.09 & 97.70 & 97.49 & 97.35 & 97.26 & 97.20 \\
\hline 34 & 0.34 & 0.01 & 0.66 & 0.79 & 0.40 & 0.36 & 0.33 & 0.33 & 0.4 & 0.44 & 0.4 & 0.5 & 0.5 & 1.18 & 0.2 & 0.07 & 0.04 & 0.02 & 0.02 \\
\hline 35 & 97.81 & 12.72 & 95.05 & 33.28 & 28.31 & 26.38 & 24.82 & 97.86 & 97.49 & 97.22 & 97.02 & 96.88 & 96.76 & 98.93 & 97.30 & 95.75 & 94.47 & 93.45 & 92.63 \\
\hline 36 & 74.69 & 94.53 & 49.93 & 2.47 & 4.64 & 6.63 & 7.89 & 74.97 & 72.99 & 71.66 & 70.71 & 70.02 & 69.49 & 73.86 & 74.92 & 75.39 & 75.66 & 75.84 & 75.96 \\
\hline 37 & 99.98 & 99.85 & 99.95 & 99.67 & $\begin{array}{l}4.04 \\
99.70\end{array}$ & $\begin{array}{l}0.03 \\
99.71\end{array}$ & 99.71 & 99.98 & 99.98 & $\begin{array}{l}11.00 \\
99.99\end{array}$ & 99.99 & $\begin{array}{l}10.02 \\
99.99\end{array}$ & $\begin{array}{l}09.49 \\
99.99\end{array}$ & 99.98 & 99.98 & 99.98 & 99.98 & 99.98 & 99.98 \\
\hline 38 & 99.99 & 99.98 & 99.95 & 89.07 & 92.71 & 95.71 & 96.71 & 99.99 & 99.99 & 99.99 & 99.99 & 99.99 & 99.99 & 99. & 99 . & 99. & 99.99 & 99.99 & 99.99 \\
\hline 39 & 100.00 & 100.00 & 100.00 & 99.93 & 99.96 & 99 & 99. & 100.00 & 100.00 & 100.00 & 100.00 & 100.00 & 100.00 & 100.00 & 100.00 & 100.00 & 100.00 & 100.00 & 100.00 \\
\hline 40 & 77.80 & 6.39 & 68.90 & 78.72 & 67.02 & 56.46 & 50.05 & 78.05 & 76.30 & 75.14 & 74.32 & 73.70 & 73.23 & 73.31 & 78.96 & 81.17 & 82.32 & 83.01 & 83.48 \\
\hline 41 & 100.00 & 100.00 & 100.00 & 99.99 & 99.99 & 100.00 & 100.00 & 100.00 & 100.00 & 100.00 & $\begin{array}{l}100.00 \\
\end{array}$ & 100.00 & 100.00 & 100.00 & 100.00 & 100.00 & 100.00 & 100.00 & 100.00 \\
\hline 42 & 100.00 & 99.95 & 99.99 & 94.73 & 94.41 & 96.64 & 97.38 & 100.00 & 100.00 & 100.00 & 99.99 & 99.99 & 99.99 & 100.00 & 100.00 & 100.00 & 100.00 & 100.00 & 100.00 \\
\hline 43 & 100.00 & 99.71 & 100.00 & 99.75 & 99.79 & & & 100 & 100 & 100.00 & 100.00 & 100.00 & 100 & & 100 & 100. & 100.00 & 100.00 & 100.00 \\
\hline 44 & 99.96 & 99.99 & 99.92 & 99.99 & 99.56 & 98.93 & 99.55 & 99.96 & 99.97 & 99.98 & 99.98 & 99.98 & 99.98 & 99.97 & 99.96 & 99.96 & 99.96 & 99.96 & 99.96 \\
\hline 45 & 86.39 & 0.38 & 97.85 & 85.76 & 71.49 & 62.16 & 56.11 & 86.82 & 83.54 & 81.09 & 79.24 & 77.82 & 76.70 & 87.53 & 86.10 & 85.54 & 85.24 & 85.05 & 84.91 \\
\hline 46 & 99.82 & 82.52 & 99.86 & 99.98 & 99.99 & 99.96 & 99.94 & $\begin{array}{l}.00 .02 \\
99.82\end{array}$ & 99.82 & 99.82 & 99.82 & 99.82 & 99.82 & 99.92 & $\begin{array}{l}0.10 \\
99.76\end{array}$ & 99.57 & $\begin{array}{l}0.24 \\
99.37\end{array}$ & 99.19 & $\begin{array}{l}9.91 .03 \\
99.03\end{array}$ \\
\hline 47 & 100.00 & 100.00 & 100.00 & 100.00 & 99.96 & 99.99 & 99.99 & 100.00 & 100.00 & 100.00 & 100.00 & 100.00 & 100.00 & 100.00 & 100.00 & 100.00 & 100.00 & 100.00 & 100.00 \\
\hline 48 & 98.66 & 99.40 & 97.60 & 99.76 & 99.89 & 99.98 & 99.96 & 98.65 & 98.69 & 98.72 & 98.75 & 98.76 & 98.77 & 99.62 & 97.90 & 93.93 & 88.11 & 81.65 & 75.52 \\
\hline
\end{tabular}


Table S7 (cont). Dependence of DP4+ with each of its $16[\mu, \sigma, v]$ parameters determined by correlating the GIAO NMR chemical shifts computed at the $\mathrm{PCM} / \mathrm{mPW} 1 \mathrm{PW} 91 / 6-31+\mathrm{G}^{* *} / / \mathrm{B} 3 \mathrm{LYP} / 6-31 \mathrm{G}^{*}$ level of theory (M3P), with $15[\mu, \sigma, v]_{0}$ parameters estimated at the M3P level and allowing the remaining one to oscillate between 6 levels. The results obtained with the matched M3P parameters are shown in grey.

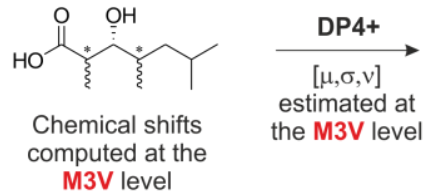

Matched

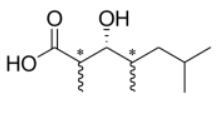

Chemical shifts
computed at the M3V level

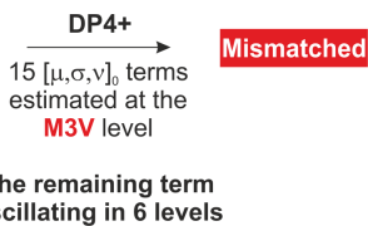

oscillating in 6 levels

\begin{tabular}{|c|c|c|c|c|c|c|c|c|c|c|c|c|c|c|c|c|c|c|c|}
\hline \multirow[b]{2}{*}{ Comp $\mathbf{N}^{\circ}$} & \multirow[b]{2}{*}{ M3P } & \multicolumn{6}{|c|}{${ }^{1} \mathbf{H} \sigma_{\mathrm{u}, \mathrm{sp} 2}$} & \multicolumn{6}{|c|}{${ }^{1} \mathbf{H} \sigma_{\mathrm{u}, \mathrm{sp} 3}$} & \multicolumn{6}{|c|}{${ }^{13} \mathrm{C} \sigma_{\mathrm{s}}$} \\
\hline & & $\mathbf{x}_{\mathrm{m}}$ & $x_{m}+1 / 5 \Delta$ & $x_{m}+2 / 5 \Delta$ & $\mathbf{x}_{\mathrm{m}}+3 / 5 \Delta$ & $x_{m}+4 / 5 \Delta$ & $\mathbf{x}_{\mathbf{M}}$ & $\mathbf{x}_{\mathrm{m}}$ & $x_{m}+1 / 5 \Delta$ & $x_{m}+2 / 5 \Delta$ & $\mathbf{x}_{\mathrm{m}}+3 / 5 \Delta$ & $\mathbf{x}_{\mathrm{m}}+4 / 5 \Delta$ & $\mathbf{x}_{\mathrm{M}}$ & $\mathbf{x}_{\mathrm{m}}$ & $\mathbf{x}_{\mathrm{m}}+1 / 5 \Delta$ & $x_{m}+2 / 5 \Delta$ & $x_{m}+3 / 5 \Delta$ & $x_{m}+4 / 5 \Delta$ & $\mathbf{x}_{\mathrm{M}}$ \\
\hline 1 & 100.00 & 100.00 & 100.00 & 100.00 & 100.00 & 100.00 & 100.00 & 100.00 & 100.00 & 100.00 & 100.00 & 100.00 & 100.00 & 100.00 & 100.00 & 100.00 & 100.00 & 100.00 & 100.00 \\
\hline 2 & 97.27 & 97.58 & 95.42 & 94.00 & 93.06 & 92.40 & 91.92 & 96.35 & 98.00 & 98.43 & 98.60 & 98.67 & 98.72 & 98.53 & 96.26 & 94.43 & 93.08 & 92.06 & 91.29 \\
\hline 3 & 100.00 & 100.00 & 100.00 & 100.00 & 100.00 & 100.00 & 100.00 & 100.00 & 100.00 & 100.00 & 100.00 & 100.00 & 100.00 & 100.00 & 100.00 & 100.00 & 100.00 & 100.00 & 100.00 \\
\hline 4 & 99.38 & 99.31 & 99.59 & 99.68 & 99.72 & 99.74 & 99.75 & 99.46 & 99.24 & 99.08 & 98.96 & 98.88 & 98.83 & 98.93 & 99.54 & 99.69 & 99.76 & 99.79 & 99.81 \\
\hline 5 & 93.40 & 93.40 & 93.40 & 93.40 & 93.40 & 93.40 & 93.40 & 95.72 & 89.61 & 85.39 & 82.90 & 81.37 & 80.38 & 91.36 & 94.28 & 95.25 & 95.71 & 95.98 & 96.14 \\
\hline 6 & 100.00 & 100.00 & 100.00 & 100.00 & 100.00 & 100.00 & 100.00 & 100.00 & 100.00 & 99.99 & 99.99 & 99.99 & 99.99 & 100.00 & 99.99 & 99.97 & 99.93 & 99.89 & 99.85 \\
\hline 7 & 98.31 & 98.31 & 98.31 & 98.31 & 98.31 & 98.31 & 98.31 & 98.68 & 97.46 & 95.96 & 94.57 & 93.43 & 92.51 & 98.36 & 98.30 & 98.28 & 98.27 & 98.26 & 98.25 \\
\hline 8 & 100.00 & 100.00 & 100.00 & 100.00 & 100.00 & 100.00 & 100.00 & 100.00 & 100.00 & 99.99 & 99.99 & 99.99 & 99.99 & 100.00 & 99.99 & 99.96 & 99.90 & 99.81 & 99.70 \\
\hline 9 & 100.00 & 100.00 & 100.00 & 100.00 & 100.00 & 100.00 & 100.00 & 100.00 & 100.00 & 100.00 & 100.00 & 100.00 & 100.00 & 100.00 & 100.00 & 100.00 & 100.00 & 100.00 & 100.00 \\
\hline 10 & 100.00 & 100.00 & 100.00 & 100.00 & 100.00 & 100.00 & 100.00 & 100.00 & 100.00 & 100.00 & 100.00 & 100.00 & 100.00 & 100.00 & 100.00 & 100.00 & 100.00 & 100.00 & 100.00 \\
\hline 11 & 100.00 & 100.00 & 100.00 & 100.00 & 100.00 & 100.00 & 100.00 & 100.00 & 100.00 & 100.00 & 100.00 & 100.00 & 100.00 & 100.00 & 100.00 & 100.00 & 99.99 & 99.96 & 99.90 \\
\hline 12 & 100.00 & 100.00 & 100.00 & 100.00 & 100.00 & 100.00 & 100.00 & 100.00 & 100.00 & 100.00 & 100.00 & 100.00 & 100.00 & 100.00 & 100.00 & 100.00 & 100.00 & 100.00 & 100.00 \\
\hline 13 & 99.86 & 99.86 & 99.85 & 99.85 & 99.85 & 99.85 & 99.85 & 99.96 & 99.37 & 97.44 & 94.31 & 90.71 & 87.19 & 99.58 & 99.91 & 99.95 & 99.96 & 99.96 & 99.96 \\
\hline 14 & 99.69 & 99.72 & 99.55 & 99.44 & 99.37 & 99.33 & 99.29 & 99.34 & 99.87 & 99.94 & 99.96 & 99.97 & 99.97 & 99.90 & 99.43 & 98.69 & 97.91 & 97.21 & 96.62 \\
\hline 15 & 96.16 & 95.82 & 97.37 & 97.86 & 98.08 & 98.21 & 98.29 & 97.65 & 93.52 & 90.33 & 88.31 & 87.02 & 86.15 & 96.09 & 96.02 & 95.63 & 95.31 & 95.07 & 94.89 \\
\hline 16 & 100.00 & 100.00 & 100.00 & 100.00 & $\begin{array}{l}300.00 \\
100.00\end{array}$ & $\begin{array}{l}30.21 \\
100.00\end{array}$ & 100.00 & 100.00 & 100.00 & 100.00 & $\begin{array}{l}0.31 \\
100.00\end{array}$ & $\begin{array}{l}0.02 \\
100.00\end{array}$ & 100.00 & 100.00 & $\begin{array}{l}700.02 \\
100.00\end{array}$ & $\begin{array}{l}100.00 \\
\end{array}$ & 100.00 & 100.00 & $\begin{array}{l}700.09 \\
100.00\end{array}$ \\
\hline 17 & 100.00 & 100.00 & 100.00 & 100.00 & 100.00 & 100.00 & 100.00 & 100.00 & 100.00 & 100.00 & 100.00 & 100.00 & 100.00 & 100.00 & 100.00 & 100.00 & 100.00 & 100.00 & 100.00 \\
\hline 18 & 36.13 & 36.13 & 36.13 & 36.13 & 36.13 & 36.13 & 36.13 & 42.40 & 30.03 & 25.41 & 23.08 & 21.69 & 20.78 & 32.06 & 37.90 & 40.12 & 41.30 & 41.96 & 42.34 \\
\hline 19 & 19.85 & 19.85 & 19.85 & 19.85 & 19.85 & 19.85 & 19.85 & 18.03 & 22.60 & 26.13 & 28.71 & 30.56 & 31.91 & 22.80 & 18.39 & 16.62 & 15.67 & 15.08 & 14.69 \\
\hline 20 & 49.27 & 49.27 & 49.27 & 49.27 & 49.27 & 49.27 & 49.27 & 49.75 & 49.24 & 49.35 & 49.39 & 49.37 & 49.34 & 44.10 & 51.55 & 54.04 & 55.26 & 56.00 & 56.50 \\
\hline 21 & 80.31 & 80.31 & 80.31 & 80.31 & 80.31 & 80.31 & 80.31 & 81.90 & 78.29 & 76.19 & 74.83 & 73.90 & 73.22 & 90.04 & 72.48 & 60.08 & 52.64 & 47.94 & 44.77 \\
\hline 22 & 92.78 & 92.78 & 92.78 & 92.78 & 92.78 & 92.78 & 92.78 & 93.81 & 91.60 & 90.63 & 90.14 & 89.86 & 89.67 & 95.66 & 90.29 & 85.47 & 81.73 & 78.91 & 76.76 \\
\hline 23 & 73.30 & 73.30 & 73.30 & 73.30 & 73.30 & 73.30 & 73.30 & 72.66 & 74.85 & 76.84 & 78.12 & 78.94 & 79.49 & 85.65 & 64.99 & 53.37 & 46.88 & 42.90 & 40.24 \\
\hline 24 & 25.95 & 25.95 & 25.95 & 25.95 & 25.95 & 25.95 & 25.95 & 28.44 & 23.50 & 21.60 & 20.62 & 20.02 & 19.63 & 13.26 & 34.52 & 46.48 & 53.14 & 57.25 & 60.00 \\
\hline 25 & 0.00 & 0.00 & 0.00 & 0.00 & 0.00 & 0.00 & 0.00 & $\begin{array}{c}2.44 \\
0.00\end{array}$ & 0.00 & 0.00 & $\begin{array}{l}0.00 \\
0.00\end{array}$ & 0.00 & 0.00 & 0.00 & 0.00 & $\begin{array}{c}4.40 \\
0.00\end{array}$ & 0.00 & 0.00 & $\begin{array}{l}0.00 \\
0.00\end{array}$ \\
\hline 26 & 99.99 & 99.99 & 99.99 & 99.99 & 99.99 & 99.99 & 99.99 & 100.00 & 99.99 & 99.98 & 99.98 & 99.97 & 99.96 & 100.00 & 99.99 & 99.99 & 99.99 & 99.99 & 99.98 \\
\hline 27 & 98.23 & 98.23 & 98.23 & 98.23 & 98.23 & 98.23 & 98.23 & 96.38 & 99.36 & 99.80 & 99.91 & 99.95 & 99.97 & 99.29 & 96.98 & 93.83 & 90.87 & 88.43 & 86.48 \\
\hline 28 & 100.00 & 100.00 & 100.00 & 100.00 & 100.00 & 100.00 & 100.00 & 100.00 & 100.00 & 100.00 & 100.00 & 100.00 & 100.00 & 100.00 & 100.00 & 99.99 & 99.99 & 99.99 & 99.98 \\
\hline 29 & 100.00 & 100.00 & 100.00 & 100.00 & 100.00 & 100.00 & 100.00 & 100.00 & 100.00 & 100.00 & 100.00 & 100.00 & 100.00 & 100.00 & 100.00 & 100.00 & 100.00 & 100.00 & 100.00 \\
\hline 30 & 100.00 & 100.00 & 100.00 & 100.00 & 100.00 & 100.00 & 100.00 & 100.00 & 100.00 & 99.99 & 99.97 & 99.93 & 99.89 & 100.00 & 100.00 & 100.00 & 100.00 & 100.00 & 100.00 \\
\hline 31 & 100.00 & 100.00 & 100.00 & 100.00 & 100.00 & 100.00 & 100.00 & 100.00 & 100.00 & 100.00 & 99.99 & 99.98 & 99.97 & 100.00 & 100.00 & 100.00 & 100.00 & 100.00 & 100.00 \\
\hline 32 & 99.30 & 99.35 & 98.98 & 98.70 & 98.48 & 98.31 & 98.18 & 98.97 & 99.52 & 99.64 & 99.69 & 99.71 & 99. & 99.48 & 99.17 & 98.84 & 98.31 & 97.60 & 96.79 \\
\hline 33 & 97.79 & 97.60 & 98. & 98.90 & 99. & & 99 . & 98. & 96.3 & 94. & 93.26 & 92. & 91. & & 95.71 & 90.07 & 84 & 80.03 & 76.35 \\
\hline 34 & 0.34 & 0.30 & 0.74 & 1.37 & 2.10 & 2.83 & 3.50 & 0.27 & 0.35 & 0.32 & 0.30 & 0.29 & 0.28 & 0.49 & 0.22 & 0.10 & 0.06 & 0.04 & 0.03 \\
\hline 35 & 97.81 & 97.64 & 98.38 & 98.56 & 98.61 & 98.63 & 98.63 & 99.25 & 92.28 & $\begin{array}{l}0.32 \\
77.89\end{array}$ & 63.61 & 52.91 & $\begin{array}{l}0.20 \\
45.44\end{array}$ & 92.37 & 98.88 & 99.52 & 99.70 & 99.78 & 99.82 \\
\hline 36 & 74.69 & 70.66 & 89.39 & 94.36 & 96.21 & 97.08 & 97.57 & 79.97 & 63.28 & 47.18 & 36.49 & 29. & 25. & 65 & 78.70 & 83.04 & 85.08 & 86.24 & 86.99 \\
\hline 37 & 99.98 & 99.98 & 99. & 99.97 & 99 & 99. & 99. & 99. & 99. & 99. & 99 . & 99. & 99. & 99. & 99. & 99.97 & 99.97 & 99.97 & 99.96 \\
\hline 38 & 99.99 & 99.99 & 99.99 & 100.00 & 100.00 & 100.00 & 100.00 & 100.00 & 99.97 & 99.92 & 99.84 & 99.74 & 99.63 & 99.99 & 99.99 & 99.99 & 99.99 & 99.99 & 99.99 \\
\hline 39 & 100.00 & 100.00 & 100.00 & 100.00 & 100.00 & 100.00 & 100.00 & 100.00 & 100.00 & 100.00 & 100.00 & 100.00 & 100.00 & 100.00 & 100.00 & 100.00 & 100.00 & 100.00 & 100.00 \\
\hline 40 & 77.80 & 78.91 & 70.99 & 65.62 & 62.08 & 59.69 & 58.01 & 85.12 & 66.57 & 54.85 & 48.06 & 43.89 & & 67.49 & 82.22 & 86.80 & 88.84 & 89.96 & 90.65 \\
\hline 41 & 100.00 & 100.00 & 100.00 & 100.00 & 100.00 & 100.00 & 100.00 & 100.00 & 100.00 & 100.00 & 100.00 & 100.00 & 100.00 & 100.00 & 100. & 100.00 & 100.00 & 100.00 & 100.00 \\
\hline 42 & 100.00 & 100.00 & 100.00 & 100.00 & 100.00 & 100.00 & 100.00 & 100.00 & 99.99 & 99.95 & 99.89 & 99.81 & 99.72 & 100.00 & 100.00 & 100.00 & 100.00 & 100.00 & 100.00 \\
\hline 43 & 100.00 & 100.00 & 100.00 & 100.00 & 100.00 & 100.00 & 100.00 & 100.00 & 100.00 & 99.99 & 99.99 & 99.98 & 99.97 & 100.00 & 100.00 & 100.00 & 100.00 & 100.00 & 100.00 \\
\hline 44 & 99.96 & 99.96 & 99.96 & 99.96 & 99.96 & 99.96 & 99.96 & 99.96 & 99.97 & 99.97 & 99.97 & 99.97 & 99.96 & 99.96 & 99.96 & 99.96 & 99.96 & 99.96 & 99.96 \\
\hline 45 & 86.39 & 86.39 & 86.39 & 86.39 & 86.39 & 86.39 & 86.39 & 92.79 & 74.60 & 61.32 & 53.78 & 49.31 & 46. & 86.2 & 86. & 86.83 & 86.98 & 87.07 & 87.12 \\
\hline 46 & 99.82 & 99.82 & & 99.82 & & 99.82 & & 99 & 99 . & 99. & 99.69 & 99 & 99 & 99. & 99. & 98.86 & 97.90 & 96.89 & 95.95 \\
\hline 47 & 100.00 & 100.00 & 100.00 & 100.00 & 100.00 & 100.00 & 100.00 & 100.00 & 100.00 & 100.00 & 100.00 & 100.00 & 100.00 & 100.00 & 100.00 & 100.00 & 100.00 & 100.00 & 100.00 \\
\hline 48 & 98.66 & 98.66 & 98.66 & 98.66 & 98.66 & 98.66 & 98.66 & 98.18 & 99.07 & 99.35 & 99.49 & 99.57 & 99.62 & 99.62 & 97.07 & 91.09 & 83.24 & 75.55 & 68.99 \\
\hline
\end{tabular}


Table S7 (cont). Dependence of DP4+ with each of its $16[\mu, \sigma, v]$ parameters determined by correlating the GIAO NMR chemical shifts computed at the $\mathrm{PCM} / \mathrm{mPW} 1 \mathrm{PW} 91 / 6-31+\mathrm{G}^{* *} / / \mathrm{B} 3 \mathrm{LYP} / 6-31 \mathrm{G}^{*}$ level of theory (M3P), with $15[\mu, \sigma, v]_{0}$ parameters estimated at the M3P level and allowing the remaining one to oscillate between 6 levels. The results obtained with the matched M3P parameters are shown in grey.

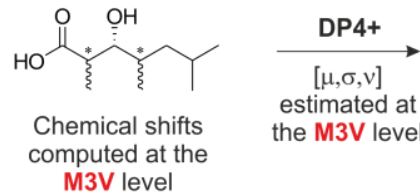

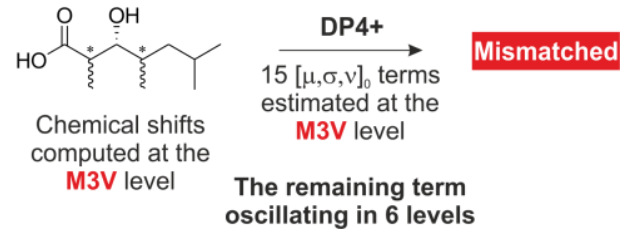

${ }^{13} \mathrm{Cv}_{\mathrm{u}, \mathrm{sp} 3}$

\begin{tabular}{|c|c|c|c|c|c|c|c|c|c|c|c|c|c|c|c|c|c|c|c|}
\hline \multirow[b]{2}{*}{ Comp $\mathbf{N}^{\circ}$} & \multirow[b]{2}{*}{ M3P } & \multicolumn{6}{|c|}{${ }^{1} \mathbf{H} \sigma_{\mathrm{s}}$} & \multicolumn{6}{|c|}{${ }^{13} \mathrm{C} \mathrm{v}$ u,sp2 } & \multicolumn{6}{|c|}{${ }^{13} \mathrm{C} v_{\mathrm{u}, \mathrm{sp} 3}$} \\
\hline & & $\mathbf{x}_{\mathrm{m}}$ & $x_{m}+1 / 5 \Delta$ & $x_{m}+2 / 5 \Delta$ & $\mathbf{x}_{\mathrm{m}}+3 / 5 \Delta$ & $x_{\mathrm{m} m}+4 / 5 \Delta$ & $\mathbf{x}_{\mathrm{M}}$ & $\mathbf{x}_{\mathrm{m}}$ & $\mathbf{x}_{\mathrm{m}}+1 / 5 \Delta$ & $x_{m}+2 / 5 \Delta$ & $\mathrm{x}_{\mathrm{m}}+3 / 5 \Delta$ & $x_{m}+4 / 5 \Delta$ & $\mathbf{x}_{\mathrm{M}}$ & $\mathbf{x}_{\mathrm{m}}$ & $\mathbf{x}_{\mathrm{m}}+1 / 5 \Delta$ & $x_{m}+2 / 5 \Delta$ & $\mathbf{x}_{\mathrm{m}}+3 / 5 \Delta$ & $x_{\mathrm{m}}+4 / 5 \Delta$ & $\mathbf{x}_{\mathrm{M}}$ \\
\hline 1 & 100.00 & 100.00 & 100.00 & 100.00 & 100.00 & 99.99 & 99.99 & 100.00 & 100.00 & 100.00 & 100.00 & 100.00 & 100.00 & 100.00 & 100.00 & 100.00 & 100.00 & 100.00 & 100.00 \\
\hline 2 & 97.27 & 97.09 & 98.48 & 98.96 & 99.18 & 99.30 & 99.38 & 97.47 & 97.26 & 97.24 & 97.27 & 97.30 & 97.34 & 89.44 & 96.63 & 98.37 & 99.01 & 99.31 & 99.48 \\
\hline 3 & 100.00 & 100.00 & 100.00 & 100.00 & 100.00 & 100.00 & 100.00 & 100.00 & 100.00 & 100.00 & 100.00 & 100.00 & 100.00 & 100.00 & 100.00 & 100.00 & 100.00 & 100.00 & 100.00 \\
\hline 4 & 99.38 & 99.30 & 99.76 & 99.86 & 99.89 & 99.91 & 99.92 & 99.25 & 99.39 & 99.45 & 99.48 & 99.50 & 99.52 & 95.82 & 99.17 & 99.68 & 99.83 & 99.89 & 99.92 \\
\hline 5 & 93.40 & 93.54 & 92.16 & 91.52 & 91.21 & 91.03 & 90.92 & 93.42 & 93.39 & 93.38 & 93.37 & 93.37 & 93.36 & 89.84 & 92.95 & 94.37 & 95.17 & 95.68 & 96.02 \\
\hline 6 & 100.00 & 100.00 & 100.00 & 99.99 & 99.99 & 99.99 & 99.99 & 100.00 & 100.00 & 100.00 & 100.00 & 100.00 & 100.00 & 99.99 & 100.00 & 100.00 & 100.00 & 100.00 & 100.00 \\
\hline 7 & 98.31 & 98.50 & 95.13 & 91.07 & 87.34 & 84.28 & 81.83 & 98.10 & 98.34 & 98.44 & 98.50 & 98.53 & 98.56 & 99.17 & 98.48 & 97.81 & 97.20 & 96.66 & 96.18 \\
\hline 8 & 100.00 & 100.00 & 99.99 & 99.99 & 99.99 & 99.99 & 99.99 & 100.00 & 100.00 & 100.00 & 100.00 & 100.00 & 100.00 & 99.98 & 100.00 & 100.00 & 100.00 & 100.00 & 100.00 \\
\hline 9 & 100.00 & 100.00 & 100.00 & 100.00 & 99.99 & 99.99 & 99.98 & 100.00 & 100.00 & 100.00 & 100.00 & 100.00 & 100.00 & 100.00 & 100.00 & 100.00 & 100.00 & 100.00 & 100.00 \\
\hline 10 & 100.00 & 100.00 & 100.00 & 100.00 & 100.00 & 100.00 & 100.00 & 100.00 & 100.00 & 100.00 & 100.00 & 100.00 & 100.00 & 100.00 & 100.00 & 100.00 & 100.00 & 100.00 & 100.00 \\
\hline 11 & 100.00 & 100.00 & 100.00 & 100.00 & 100.00 & 100.00 & 100.00 & 100.00 & 100.00 & 100.00 & 100.00 & 100.00 & 100.00 & 100.00 & 100.00 & 100.00 & 100.00 & 100.00 & 100.00 \\
\hline 12 & 100.00 & 100.00 & 100.00 & 100.00 & 100.00 & 100.00 & 100.00 & 100.00 & 100.00 & 100.00 & 100.00 & 100.00 & 100.00 & 100.00 & 100.00 & 100.00 & 100.00 & 100.00 & 100.00 \\
\hline 13 & 99.86 & 99.87 & 99.68 & 99.47 & 99.29 & 99.13 & 99.01 & 99.72 & 99.87 & 99.93 & 99.96 & 99.97 & 99.98 & 99.88 & 99.86 & 99.85 & 99.84 & 99.84 & 99.83 \\
\hline 14 & 99.69 & 99.70 & 99.64 & 99.62 & 99.61 & 99.60 & 99.59 & 99.67 & 99.70 & 99.71 & 99.72 & 99.73 & 99.74 & 99.01 & 99.64 & 99.79 & 99.86 & 99.89 & 99.91 \\
\hline 15 & 96.16 & 95.72 & 98.45 & 99.03 & 99.26 & 99.37 & 99.44 & 94.48 & 96.34 & 97.01 & 97.35 & 97.55 & 97.69 & 94.98 & 96.02 & 96.45 & 96.69 & 96.83 & 96.94 \\
\hline 16 & 100.00 & 100.00 & $\begin{array}{l}30.43 \\
99.99\end{array}$ & 99.95 & 99.86 & 99.72 & 99.54 & $\begin{array}{l}3.40 \\
100.00\end{array}$ & 100.00 & 100.00 & 100.00 & 100.00 & 100.00 & 100.00 & $\begin{array}{l}700.02 \\
100.00\end{array}$ & 100.00 & 100.00 & $\begin{array}{l}700.00 \\
100.00\end{array}$ & 100.00 \\
\hline 17 & 100.00 & 100.00 & 100.00 & 100.00 & 100.00 & 100.00 & 100.00 & 100.00 & 100.00 & 100.00 & 100.00 & 100.00 & 100.00 & 100.00 & 100.00 & 100.00 & 100.00 & 100.00 & 100.00 \\
\hline 18 & 36.13 & 38.24 & 21.51 & 16.02 & 13.51 & 12.11 & 11.23 & 37.75 & 35.86 & 34.57 & 33.66 & 33.00 & 32.50 & 42.67 & 36.98 & 34.24 & 32.64 & 31.60 & 30.86 \\
\hline $\begin{array}{l}18 \\
19\end{array}$ & 19.85 & 20.14 & $\begin{array}{l}21.31 \\
18.99\end{array}$ & $\begin{array}{l}10.02 \\
19.83\end{array}$ & 20.73 & 21.45 & $\begin{array}{l}11.23 \\
21.99\end{array}$ & 20.10 & $\begin{array}{l}3.80 \\
19.82\end{array}$ & $\begin{array}{l}3.57 \\
19.72\end{array}$ & $\begin{array}{l}39.00 \\
19.67\end{array}$ & 19.65 & 19.64 & $\begin{array}{l}4.01 \\
19.67\end{array}$ & $\begin{array}{l}50.98 \\
19.80\end{array}$ & $\begin{array}{l}3.24 \\
19.99\end{array}$ & 20.16 & 20.28 & $\begin{array}{l}20.00 \\
20.39\end{array}$ \\
\hline 20 & 49.27 & 48.69 & 53.76 & 55.78 & 56.80 & 57.42 & 57.82 & 48.99 & 49.30 & 49.38 & 49.40 & 49.41 & 49.40 & 46.35 & 48.88 & 50.15 & 50.90 & 51.41 & 51.77 \\
\hline 21 & 80.31 & $\begin{array}{l}40.099 \\
79.98\end{array}$ & 82.47 & 83.23 & 83.56 & 83.74 & 83.85 & 77.31 & 80.70 & 82.35 & 83.32 & 83.96 & 84.42 & 83.25 & $\begin{array}{l}40.00 \\
80.74\end{array}$ & 79.31 & 78.40 & 77.76 & 77.29 \\
\hline 22 & 92.78 & 92.93 & 91.61 & 91.07 & 90.80 & 90.64 & 90.53 & 91.28 & 92.96 & 93.66 & 94.05 & 94.29 & 94.46 & 93.07 & 92.81 & 92.74 & 92.71 & 92.70 & 92.69 \\
\hline 23 & 73.30 & 72.86 & 78.35 & 81.66 & 83.54 & 84.69 & 85.44 & 74.40 & 73.11 & 72.17 & 71.49 & 70.98 & 70.59 & 71.80 & 73.12 & 73.70 & 74.03 & 74.24 & 74.38 \\
\hline 24 & 25.95 & 26.85 & 18.88 & 15.62 & 13.96 & 12.99 & 12.36 & 25.00 & 26.10 & 26.87 & 27.41 & 27.80 & 28.10 & 25.68 & 25.87 & 26.16 & 26.38 & 26.56 & 26.69 \\
\hline 25 & 0.00 & 0.00 & 0.00 & 0.00 & 0.00 & 0.00 & 0.00 & 0.00 & 0.00 & 0.00 & 0.00 & 0.00 & 0.00 & 0.00 & 0.00 & 0.00 & 0.00 & 0.00 & 0.00 \\
\hline 26 & 99.99 & 99.99 & 100.00 & 100.00 & 100.00 & 100.00 & 100.00 & 99.99 & 99.99 & 99.99 & 99.99 & 99.99 & 99.99 & 99.95 & 99.99 & 100.00 & 100.00 & 100.00 & 100.00 \\
\hline 27 & 98.23 & 98.34 & 97.08 & 96.28 & 95.77 & 95.41 & 95.15 & 98.23 & 98.23 & 98.23 & 98.23 & 98.23 & 98.23 & 88.26 & 97.57 & 99.21 & 99.66 & 99.82 & 99.89 \\
\hline 28 & 100.00 & 100.00 & 100.00 & 100.00 & 100.00 & 99.99 & 99.99 & 100.00 & 100.00 & 100.00 & 100.00 & 100.00 & 100.00 & $\begin{array}{l}0.20 \\
99.99\end{array}$ & 100.00 & 100.00 & 100.00 & $\begin{array}{l}100.00 \\
100.00\end{array}$ & 100.00 \\
\hline 29 & 100.00 & 100.00 & 100.00 & 100.00 & 100.00 & 100.00 & 100.00 & 100.00 & 100.00 & 100.00 & 100.00 & 100.00 & 100.00 & 100.00 & 100.00 & 100.00 & 100.00 & 100.00 & 100.00 \\
\hline 30 & 100.00 & 100.00 & 100.00 & 100.00 & 100.00 & 99.99 & 99.99 & 100.00 & 100.00 & 100.00 & 100.00 & 100.00 & 100.00 & 100.00 & 100.00 & 100.00 & 100.00 & 100.00 & 100.00 \\
\hline 31 & 100.00 & 100.00 & 100.00 & 99.98 & 99.95 & 99.91 & 99.85 & 100.00 & 100.00 & 100.00 & 100.00 & 100.00 & 100.00 & 100.00 & 100.00 & 100.00 & 100.00 & 100.00 & 100.00 \\
\hline 32 & 99.30 & 99.34 & 98.80 & 98.37 & 98.02 & 97.75 & 97.54 & 99.41 & 99.28 & 99.21 & 99.17 & 99.14 & 99.12 & 98.51 & 99.22 & 99.45 & 99.56 & 99.63 & 99.67 \\
\hline 33 & 97.79 & 97.69 & $\begin{array}{l}98.00 \\
98.58\end{array}$ & $\begin{array}{l}7.37 \\
98.93\end{array}$ & $\begin{array}{l}9.02 \\
99.10\end{array}$ & 99.20 & 99.27 & 97.46 & 97.83 & 98.00 & 98.09 & 98.15 & 98.19 & 97.65 & 97.77 & 97.84 & 97.89 & 97.93 & 97.96 \\
\hline 34 & 0.34 & 0.30 & 1.11 & 2.69 & 4.89 & 7.36 & 9.83 & 0.36 & 0.33 & 0.32 & 0.31 & 0.30 & 0.3 & 0.1 & 0.29 & 0.48 & 0.66 & 0.84 & 1.00 \\
\hline 35 & 97.81 & 97.80 & 97.83 & 97.75 & 97.65 & 97.55 & 97.45 & 97.68 & 97.83 & 97.88 & 97.91 & 97.92 & 97.93 & 97.14 & 97.73 & 97.99 & 98.13 & 98.21 & 98.28 \\
\hline 36 & 74.69 & 75.15 & 66.12 & 56.31 & 48.68 & 43.22 & 39.35 & 73.68 & 74.82 & 75.35 & 75.66 & 75.86 & 76. & 74.68 & 74.69 & 74.71 & 74.73 & 74.74 & 74.75 \\
\hline 37 & 99.98 & 99.98 & 99.97 & 99.95 & 99.94 & 99.92 & 99.91 & 99.98 & 99.98 & 99.98 & 99.98 & 99.98 & 99.98 & 99.98 & 99.98 & 99.98 & 99.98 & 99.98 & 99.98 \\
\hline 38 & 99.99 & 99.99 & 99.97 & 99.92 & 99.84 & 99.75 & 99.66 & 99.99 & 99.99 & 99.99 & 99.99 & 99.99 & 99.99 & 99.99 & 99.99 & 99.99 & 99.99 & 99.99 & 99.99 \\
\hline 39 & 100.00 & 100.00 & 100.00 & 100.00 & 100.00 & 99.99 & 99.99 & 100.00 & 100.00 & 100.00 & 100.00 & 100.00 & 100.00 & 100.00 & 100.00 & 100.00 & 100.00 & 100.00 & 100.00 \\
\hline 40 & 77.80 & 77.73 & 79.23 & 80 & & 83. & 83. & 77.5 & 77.8 & 77.92 & 77.96 & 77. & 78. & 78.54 & 77.89 & 77.62 & 77.48 & 77.40 & 77.34 \\
\hline 41 & 100.00 & 100.00 & 100.00 & 100.00 & 100.00 & 100.00 & 100.00 & 100.00 & 100.00 & 100.00 & 100.00 & 100.00 & 100.00 & 100.00 & 100.00 & 100.00 & 100.00 & 100.00 & 100.00 \\
\hline 42 & 100.00 & 100.00 & 99.99 & 99.97 & 99.94 & 99.91 & 99.88 & 100.00 & 100.00 & 100.00 & 100.00 & 100.00 & 100.00 & 100.00 & 100.00 & 100.00 & 100.00 & 100.00 & 100.00 \\
\hline 43 & 100.00 & 100.00 & 100.00 & 99.99 & 99.98 & 99.98 & 99.97 & 100.00 & 100.00 & 100.00 & 100.00 & 100.00 & 100.00 & 100.00 & 100.00 & 100.00 & 100.00 & 100.00 & 100.00 \\
\hline 44 & 99.96 & 99.97 & 99.58 & 98.30 & 96.01 & 93.03 & 89.76 & 99.97 & 99.96 & 99.96 & 99.96 & 99.96 & 99.96 & 99. & 99.96 & 99.96 & 99.97 & 99.97 & 99.97 \\
\hline $\begin{array}{l}44 \\
45\end{array}$ & 86.39 & 85.64 & 91.98 & $\begin{array}{l}98.30 \\
94.29\end{array}$ & 95.36 & 95.95 & $\begin{array}{l}89.31 \\
96.31\end{array}$ & 85. & 86.55 & 87. & 87.53 & 87. & 87. & 86.8 & 86.46 & 86.23 & 86.07 & 85.96 & 85.88 \\
\hline 46 & 99.82 & 99.82 & 99.86 & 99.90 & 99.92 & 99.93 & 99.93 & 99.82 & 99.82 & 99.82 & 99.82 & 99.82 & 99.82 & 99.67 & 99.80 & 99.86 & 99.89 & 99.91 & 99.92 \\
\hline $\begin{array}{l}40 \\
47\end{array}$ & 100.00 & 100.00 & 100.00 & 100.00 & 100.00 & 100.00 & 100.00 & 100.00 & 100.00 & $\begin{array}{l}100.00 \\
100.00\end{array}$ & $\begin{array}{l}100.00 \\
100.02\end{array}$ & $\begin{array}{l}100.00 \\
100.00\end{array}$ & $\begin{array}{l}100.00 \\
100.00\end{array}$ & 100.00 & $\begin{array}{l}100.00 \\
100\end{array}$ & $\begin{array}{l}100.00 \\
100\end{array}$ & 100.00 & 100.00 & 100.00 \\
\hline 48 & 98.66 & 98.60 & 99.23 & 99.53 & 99.69 & 99.77 & 99.82 & 98.70 & 98.65 & 98.62 & 98.60 & 98.58 & 98.57 & 94.19 & 98.30 & 99.26 & 99.60 & 99.75 & 99.83 \\
\hline
\end{tabular}


Table S7 (cont). Dependence of DP4+ with each of its $16[\mu, \sigma, v]$ parameters determined by correlating the GIAO NMR chemical shifts computed at the $\mathrm{PCM} / \mathrm{mPW} 1 \mathrm{PW} 91 / 6-31+\mathrm{G}^{* *} / / \mathrm{B} 3 \mathrm{LYP} / 6-31 \mathrm{G}^{*}$ level of theory (M3P), with $15[\mu, \sigma, v]_{0}$ parameters estimated at the M3P level and allowing the remaining one to oscillate between 6 levels. The results obtained with the matched M3P parameters are shown in grey.

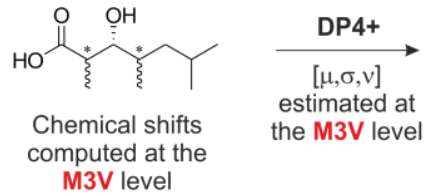

Matched

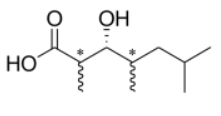

Chemical shifts
computed at the M3V level

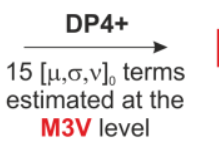

The remaining term oscillating in 6 levels

\begin{tabular}{|c|c|c|c|c|c|c|c|c|c|c|c|c|c|c|c|c|c|c|c|}
\hline \multirow[b]{2}{*}{ Comp $\mathbf{N}^{\circ}$} & \multirow[b]{2}{*}{ M3P } & \multicolumn{6}{|c|}{${ }^{1} H v_{u, s p 2}$} & \multicolumn{6}{|c|}{${ }^{1} \mathbf{H} \mathbf{v}_{\mathrm{u}, \mathrm{sp} 3}$} & \multicolumn{6}{|c|}{${ }^{13} \mathrm{C} \mathrm{v}{ }_{\mathrm{s}}$} \\
\hline & & $\mathbf{x}_{\mathrm{m}}$ & $x_{m}+1 / 5 \Delta$ & $x_{m}+2 / 5 \Delta$ & $\mathbf{x}_{\mathrm{m}}+3 / 5 \Delta$ & $x_{m}+4 / 5 \Delta$ & $\mathbf{x}_{\mathbf{M}}$ & $\mathbf{x}_{\mathrm{m}}$ & $x_{m}+1 / 5 \Delta$ & $x_{m}+2 / 5 \Delta$ & $x_{m}+3 / 5 \Delta$ & $x_{m}+4 / 5 \Delta$ & $\mathbf{x}_{\mathrm{M}}$ & $\mathbf{x}_{\mathrm{m}}$ & $x_{m}+1 / 5 \Delta$ & $x_{m}+2 / 5 \Delta$ & $\mathbf{x}_{\mathrm{m} t}+3 / 5 \Delta$ & $x_{m}+4 / 5 \Delta$ & $\mathbf{x}_{\mathbf{M}}$ \\
\hline 1 & 100.00 & 100.00 & 100.00 & 100.00 & 100.00 & 100.00 & 100.00 & 100.00 & 100.00 & 100.00 & 100.00 & 100.00 & 100.00 & 100.00 & 100.00 & 100.00 & 100.00 & 100.00 & 100.00 \\
\hline 2 & 97.27 & 97.09 & 97.41 & 97.53 & 97.60 & 97.64 & 97.67 & 97.27 & 97.36 & 97.54 & 97.73 & 97.92 & 98.09 & 96.70 & 97.04 & 97.25 & 97.39 & 97.50 & 97.58 \\
\hline 3 & 100.00 & 100.00 & 100.00 & 100.00 & 100.00 & 100.00 & 100.00 & 100.00 & 100.00 & 100.00 & 100.00 & 100.00 & 100.00 & 100.00 & 100.00 & 100.00 & 100.00 & 100.00 & 100.00 \\
\hline 4 & 99.38 & 99.42 & 99.33 & 99.28 & 99.25 & 99.23 & 99.22 & 99.38 & 99.47 & 99.53 & 99.57 & 99.60 & 99.62 & 99.53 & 99.44 & 99.38 & 99.34 & 99.30 & 99.27 \\
\hline 5 & 93.40 & 93.40 & 93.40 & 93.40 & 93.40 & 93.40 & 93.40 & 93.40 & 93.71 & 93.65 & 93.44 & 93.16 & 92.85 & 93.88 & 93.59 & 93.42 & 93.30 & 93.23 & 93.17 \\
\hline 6 & 100.00 & 100.00 & 100.00 & 100.00 & 100.00 & 100.00 & 100.00 & 100.00 & 100.00 & 100.00 & 100.00 & 100.00 & 100.00 & 99.99 & 99.99 & 100.00 & 100.00 & 100.00 & 100.00 \\
\hline 7 & 98.31 & 98.31 & 98.31 & 98.31 & 98.31 & 98.31 & 98.31 & 98.31 & 99.05 & 99.37 & 99.54 & 99.64 & 99.71 & 98.39 & 98.35 & 98.32 & 98.29 & 98.28 & 98.27 \\
\hline 8 & 100.00 & 100.00 & 100.00 & 100.00 & 100.00 & 100.00 & 100.00 & 100.00 & 100.00 & 100.00 & 100.00 & 100.00 & 100.00 & 99.99 & 99.99 & 100.00 & 100.00 & 100.00 & 100.00 \\
\hline 9 & 100.00 & 100.00 & 100.00 & 100.00 & 100.00 & 100.00 & 100.00 & 100.00 & 100.00 & 100.00 & 100.00 & 100.00 & 100.00 & 100.00 & 100.00 & 100.00 & 100.00 & 100.00 & 100.00 \\
\hline 10 & 100.00 & 100.00 & 100.00 & 100.00 & 100.00 & 100.00 & 100.00 & 100.00 & 100.00 & 100.00 & 100.00 & 100.00 & 100.00 & 100.00 & 100.00 & 100.00 & 100.00 & 100.00 & 100.00 \\
\hline 11 & 100.00 & 100.00 & 100.00 & 100.00 & 100.00 & 100.00 & 100.00 & 100.00 & 100.00 & 100.00 & 100.00 & 100.00 & 100.00 & 100.00 & 100.00 & 100.00 & 100.00 & 100.00 & 100.00 \\
\hline 12 & 100.00 & 100.00 & 100.00 & 100.00 & 100.00 & 100.00 & 100.00 & 100.00 & 100.00 & 100.00 & 100.00 & 100.00 & 100.00 & 100.00 & 100.00 & 100.00 & 100.00 & 100.00 & 100.00 \\
\hline 13 & 99.86 & 99.86 & 99.86 & 99.86 & 99.86 & 99.86 & 99.86 & 99.86 & 99.95 & 99.98 & 99.99 & 99.99 & 99.99 & 99.86 & 99.85 & 99.86 & 99.86 & 99.87 & 99.88 \\
\hline 14 & 99.69 & 99.67 & 99.71 & 99.72 & 99.73 & 99.74 & 99.74 & 99.69 & 99.51 & 99.33 & 99.16 & 99.00 & 98.85 & 99.36 & 99.58 & 99.68 & 99.74 & 99.78 & 99.80 \\
\hline 15 & 96.16 & 96.41 & 95.93 & 95.69 & 95.54 & 95.45 & 95.38 & 96.16 & 96.44 & 96.47 & 96.40 & 96.29 & 96.16 & 94.75 & 95.55 & 96.09 & 96.48 & 96.77 & 96.99 \\
\hline 16 & 100.00 & $\begin{array}{l}5.41 \\
100.00\end{array}$ & 100.00 & 100.00 & 100.00 & 100.00 & 100.00 & 100.00 & $\begin{array}{l}5.44 \\
100.00\end{array}$ & $\begin{array}{l}700.00 \\
100.00\end{array}$ & 100.00 & 100.00 & $\begin{array}{l}100.00 \\
100\end{array}$ & 100.00 & 100.00 & 100.00 & $\begin{array}{l}300.00 \\
100.00\end{array}$ & 100.00 & 100.00 \\
\hline 17 & 100.00 & 100.00 & 100.00 & 100.00 & 100.00 & 100.00 & 100.00 & 100.00 & 100.00 & 100.00 & 100.00 & 100.00 & 100.00 & 100.00 & 100.00 & 100.00 & 100.00 & 100.00 & 100.00 \\
\hline 18 & 36.13 & 36.13 & 36.13 & 36.13 & 36.13 & 36.13 & 36.13 & 36.13 & 37.12 & 37.58 & 37.83 & 37.98 & 38.07 & 43.18 & 39.19 & 36.45 & 34.49 & 33.01 & 31.87 \\
\hline 19 & 19.85 & 19.85 & 19.85 & 19.85 & 19.85 & 19.85 & 19.85 & 19.85 & 16.50 & 14.22 & 12.59 & 11.39 & 10.47 & 19.10 & 19.53 & 19.81 & 20.02 & 20.18 & 20.31 \\
\hline 20 & 49.27 & 49.27 & 49.27 & 49.27 & 49.27 & 49.27 & 49.27 & 49.27 & 48.18 & 47.52 & $\begin{array}{l}47.09 \\
\end{array}$ & 46.78 & 46.55 & $\begin{array}{l}47.48 \\
\end{array}$ & 48.38 & 49.17 & 49.82 & 50.35 & 50.78 \\
\hline 21 & 80.31 & 80.31 & 80.31 & 80.31 & 80.31 & 80.31 & 80.31 & 80.31 & 80.52 & 80.55 & 80.52 & 80.47 & 80.43 & 77.12 & 79.18 & 80.21 & 80.80 & 81.18 & 81.43 \\
\hline 22 & 92.78 & 92.78 & 92.78 & 92.78 & 92.78 & 92.78 & 92.78 & 92.78 & 92.82 & 92.77 & 92.70 & 92.63 & 92.57 & 90.89 & 92.06 & 92.71 & 93.13 & 93.42 & 93.64 \\
\hline 23 & 73.30 & 73.30 & 73.30 & 73.30 & 73.30 & 73.30 & 73.30 & 73.30 & 70.18 & 67.81 & 65.97 & 64.50 & 63.31 & 70.56 & 72.32 & 73.21 & 73.73 & 74.08 & 74.32 \\
\hline 24 & 25.95 & 25.95 & 25.95 & 25.95 & 25.95 & 25.95 & 25.95 & 25.95 & 26.63 & 27.07 & 27.38 & 27.61 & 27.78 & 27.27 & 26.31 & 25.97 & 25.84 & 25.79 & 25.79 \\
\hline 25 & 0.00 & 0.00 & 0.00 & 0.00 & 0.00 & 0.00 & 0.00 & 0.00 & 0.00 & 0.00 & 0.00 & 0.00 & 0.00 & 0.00 & 0.00 & 0.00 & $\begin{array}{c}2.04 \\
0.00\end{array}$ & 0.00 & 0.00 \\
\hline 26 & 99.99 & 99.99 & 99.99 & 99.99 & 99.99 & 99.99 & 99.99 & 99.99 & 100.00 & 100.00 & 100.00 & 100.00 & 100.00 & 99. & 9 & 99.9 & 99. & 99.99 & 99.99 \\
\hline 27 & 98.23 & 98.23 & 98.23 & 98.23 & 98.23 & 98.23 & 98.23 & 98.23 & 91.53 & 74.46 & 49.64 & 28.23 & 15.13 & 96.46 & 97.61 & 98.17 & 98.49 & 98.70 & 98.84 \\
\hline 28 & 100.00 & 100.00 & 100.00 & 100.00 & 100.00 & 100.00 & 100.00 & 100.00 & 100.00 & 100.00 & 100.00 & 100.00 & 100.00 & 100.00 & 100.00 & 100.00 & 100.00 & 100.00 & 100.00 \\
\hline 29 & 100.00 & 100.00 & 100.00 & 100.00 & 100.00 & 100.00 & 100.00 & 100.00 & 100.00 & 100.00 & 100.00 & 100.00 & 100.00 & 100.00 & 100.00 & 100.00 & 100.00 & 100.00 & 100.00 \\
\hline 30 & 100.00 & 100.00 & 100.00 & 100.00 & 100.00 & 100.00 & 100.00 & 100.00 & 100.00 & 100.00 & 100.00 & 100.00 & 100.00 & 100.00 & 100.00 & 100.00 & 100.00 & 100.00 & 100.00 \\
\hline 31 & 100.00 & 100.00 & 100.00 & 100.00 & 100.00 & 100.00 & 100.00 & 100.00 & 100.00 & 100.00 & 100.00 & 100.00 & 100.00 & 100.00 & 100.00 & 100.00 & 100.00 & 100.00 & 100.00 \\
\hline 32 & 99.30 & 99.18 & 99.42 & 99.55 & 99.63 & 99.68 & 99.72 & 99.30 & 99.27 & 99.25 & 99.24 & 99.23 & 99.22 & 99.29 & 99.30 & 99.30 & 99.29 & 99.29 & 99.28 \\
\hline 33 & 97.79 & 98.17 & 97.30 & 96. & 95.88 & 95. & 94. & 97.79 & 98.1 & 98. & 98.49 & 98. & 98. & 96. & 97. & 97.74 & 98.05 & 98.25 & 98.39 \\
\hline 34 & 0.34 & 0.64 & 0.16 & 0.06 & 0.03 & 0.01 & 0.01 & 0.34 & 0.25 & 0.19 & 0.15 & 0.12 & 0.11 & 0.15 & 0.24 & 0.33 & 0.40 & 0.47 & 0.52 \\
\hline 35 & 97.81 & $\begin{array}{l}0.04 \\
97.74\end{array}$ & 97.95 & 98.17 & 98.35 & 98.48 & 98.58 & 97.81 & 99.05 & 99.46 & 99.64 & 99.74 & 99.79 & 98.49 & 98.12 & 97.85 & $\begin{array}{l}0.40 \\
97.63\end{array}$ & 97.46 & 97.31 \\
\hline 36 & 74.69 & 82.28 & 64.54 & 50.31 & 40.20 & 33.15 & 28.1 & 74.69 & 88.02 & 93.55 & 96.09 & 97.41 & 98. & 76.5 & 75 & 74.7 & 74.34 & 74.05 & 73.83 \\
\hline 37 & 99.98 & 99.97 & 99.99 & 100.00 & 100.00 & 100.00 & 100. & 99. & 99. & 99. & 99. & 99. & 99. & 99 . & 99. & 99.9 & 99. & 99.98 & 99.98 \\
\hline 38 & 99.99 & 99.99 & 99.99 & 99.99 & 99.99 & 99.99 & 99.99 & 99.99 & 100.00 & 100.00 & 100.00 & 100.00 & 100.00 & 99.99 & 99.99 & 99.99 & 99.99 & 99.99 & 99.99 \\
\hline 39 & 100.00 & 100.00 & 100.00 & 100.00 & 100.00 & 100.00 & 100.00 & 100.00 & 100.00 & 100.00 & 100.00 & 100.00 & 100.00 & 100.00 & 100.00 & 100.00 & 100.00 & 100.00 & 100.00 \\
\hline 40 & 77.80 & 74.39 & 81.09 & 84.57 & 86.66 & 88.04 & 89.02 & 77.80 & 82.16 & 84.57 & 86.08 & 87.12 & & 80.84 & & 77.93 & 77.16 & 76.61 & 76.19 \\
\hline 41 & 100.00 & 100.00 & 100.00 & 100.00 & 100.00 & 100.00 & 100.00 & 100.00 & 100.00 & 100.00 & 100.00 & 100.00 & 100.00 & 100.00 & 100.00 & 100.00 & 100.00 & 100.00 & 100.00 \\
\hline 42 & 100.00 & 100.00 & 100.00 & 100.00 & 99.99 & & 99.99 & 100.00 & 100.00 & 100.00 & 100.00 & 100.00 & 100.00 & 100.00 & 100.00 & 100.00 & 100.00 & 100.00 & 100.00 \\
\hline 43 & 100.00 & 100.00 & 100.00 & 100.00 & 100.00 & 100.00 & 100.00 & 100.00 & 100.00 & 100.00 & 100.00 & 100.00 & 100.00 & 100.00 & 100.00 & 100.00 & 100.00 & 100.00 & 100.00 \\
\hline 44 & 99.96 & 99.96 & 99.96 & 99.96 & 99.96 & 99.96 & 99.96 & 99.96 & 99.98 & 99.99 & 99.99 & 100.00 & 100.00 & 99.96 & 99.96 & 99.96 & 99.96 & 99.97 & 99.97 \\
\hline 45 & 86.39 & 86.39 & 86.39 & 86.39 & 86.39 & 86.39 & 86.39 & 86.39 & 88.27 & 88.94 & 89.15 & 89.15 & 89.05 & 87.18 & 86.74 & 86.43 & 86.19 & 86.01 & 85.87 \\
\hline 46 & 99.82 & 99.82 & 99.82 & & & & & & 99. & 99. & 99. & 99.58 & 99 & 99. & 99. & 99.81 & 99.8 & 99.89 & 99.91 \\
\hline 47 & 100.00 & 100.00 & 100.00 & 100.00 & 100.00 & 100.00 & 100.00 & 100.00 & 100.00 & 100.00 & 100.00 & 100.00 & 100.00 & 100.00 & 100.00 & 100.00 & 100.00 & 100.00 & 100.00 \\
\hline 48 & 98.66 & 98.66 & 98.66 & 98.66 & 98.66 & 98.66 & 98.66 & 98.66 & 97.76 & 96.54 & 94.95 & 92.94 & 90.48 & 94.64 & 97.45 & 98.56 & 99.08 & 99.37 & 99.53 \\
\hline
\end{tabular}


Table S7 (cont). Dependence of DP4+ with each of its $16[\mu, \sigma, v]$ parameters determined by correlating the GIAO NMR chemical shifts computed at the $\mathrm{PCM} / \mathrm{mPW} 1 \mathrm{PW} 91 / 6-31+\mathrm{G}^{* *} / / \mathrm{B} 3 \mathrm{LYP} / 6-31 \mathrm{G}^{*}$ level of theory (M3P), with $15[\mu, \sigma, v]_{0}$ parameters estimated at the M3P level and allowing the remaining one to oscillate between 6 levels. The results obtained with the matched M3P parameters are shown in grey.

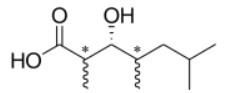

Chemical shifts computed at the M3V level

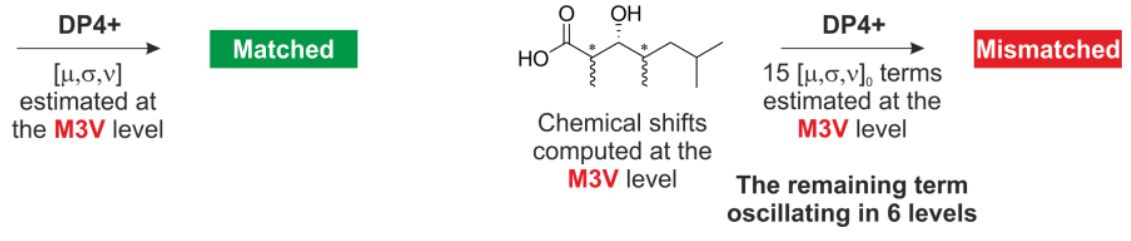

\begin{tabular}{|c|c|c|c|c|c|c|c|}
\hline \multirow[b]{2}{*}{ Comp $\mathbf{N}^{\circ}$} & \multirow[b]{2}{*}{ M3P } & \multicolumn{6}{|c|}{${ }^{1} H v_{s}$} \\
\hline & & $\mathbf{x}_{\mathrm{m}}$ & $\mathbf{x}_{\mathrm{m}}+\mathbf{1} / \mathbf{5 \Delta}$ & $x_{m}+2 / 5 \Delta$ & $\mathbf{x}_{\mathrm{m}}+3 / 5 \Delta$ & $\mathbf{x}_{\mathrm{m}}+\mathbf{4} / \mathbf{5 \Delta}$ & $\mathbf{x}_{\mathrm{M}}$ \\
\hline 1 & 100.00 & 100.00 & 100.00 & 100.00 & 100.00 & 100.00 & 100.00 \\
\hline 2 & 97.27 & 97.27 & 96.28 & 95.22 & 94.13 & 93.02 & 91.90 \\
\hline 3 & 100.00 & 100.00 & 100.00 & 100.00 & 100.00 & 100.00 & 100.00 \\
\hline 4 & 99.38 & 99.38 & 99.20 & 99.04 & 98.90 & 98.78 & 98.68 \\
\hline 5 & 93.40 & 93.40 & 93.40 & 93.34 & 93.25 & 93.14 & 93.03 \\
\hline 6 & 100.00 & 100.00 & 100.00 & 100.00 & 100.00 & 100.00 & 100.00 \\
\hline 7 & 98.31 & 98.31 & 98.93 & 99.25 & 99.44 & 99.56 & 99.64 \\
\hline 8 & 100.00 & 100.00 & 100.00 & 100.00 & 100.00 & 100.00 & 100.00 \\
\hline 9 & 100.00 & 100.00 & 100.00 & 100.00 & 100.00 & 100.00 & 100.00 \\
\hline 10 & 100.00 & 100.00 & 100.00 & 100.00 & 100.00 & 100.00 & 100.00 \\
\hline 11 & 100.00 & 100.00 & 100.00 & 100.00 & 100.00 & 100.00 & 100.00 \\
\hline 12 & 100.00 & 100.00 & 100.00 & 100.00 & 100.00 & 100.00 & 100.00 \\
\hline 13 & 99.86 & 99.86 & 99.90 & 99.93 & 99.95 & 99.96 & 99.96 \\
\hline 14 & 99.69 & 99.69 & 99.68 & 99.67 & 99.65 & 99.64 & 99.63 \\
\hline 15 & 96.16 & 96.16 & 95.31 & 94.59 & 93.99 & 93.47 & 93.02 \\
\hline 16 & 100.00 & 100.00 & 100.00 & 100.00 & 100.00 & 100.00 & 100.00 \\
\hline 17 & 100.00 & 100.00 & 100.00 & 100.00 & 100.00 & 100.00 & 100.00 \\
\hline 18 & 36.13 & 36.13 & 38.20 & 39.45 & 40.27 & 40.84 & 41.25 \\
\hline 19 & 19.85 & 19.85 & 16.88 & 14.61 & 12.87 & 11.50 & 10.42 \\
\hline 20 & 49.27 & 49.27 & 48.72 & 48.41 & 48.22 & 48.10 & 48.02 \\
\hline 21 & 80.31 & 80.31 & 80.43 & 80.55 & 80.65 & 80.74 & 80.81 \\
\hline 22 & 92.78 & 92.78 & 92.68 & 92.53 & 92.37 & 92.21 & 92.06 \\
\hline 23 & 73.30 & 73.30 & 68.74 & 64.88 & 61.64 & 58.90 & 56.58 \\
\hline 24 & 25.95 & 25.95 & 28.63 & 30.71 & 32.38 & 33.73 & 34.86 \\
\hline 25 & 0.00 & 0.00 & 0.00 & 0.00 & 0.00 & 0.00 & 0.00 \\
\hline 26 & 99.99 & 99.99 & 99.99 & 99.99 & 99.99 & 99.99 & 99.99 \\
\hline 27 & 98.23 & 98.23 & 98.34 & 98.41 & 98.46 & 98.50 & 98.53 \\
\hline 28 & 100.00 & 100.00 & 100.00 & 100.00 & 100.00 & 100.00 & 100.00 \\
\hline 29 & 100.00 & 100.00 & 100.00 & 100.00 & 100.00 & 100.00 & 100.00 \\
\hline 30 & 100.00 & 100.00 & 100.00 & 100.00 & 100.00 & 100.00 & 100.00 \\
\hline 31 & 100.00 & 100.00 & 100.00 & 100.00 & 100.00 & 100.00 & 100.00 \\
\hline 32 & 99.30 & 99.30 & 99.44 & 99.53 & 99.59 & 99.64 & 99.68 \\
\hline 33 & 97.79 & 97.79 & 97.17 & 96.54 & 95.91 & 95.30 & 94.70 \\
\hline 34 & 0.34 & 0.34 & 0.10 & 0.04 & 0.01 & 0.01 & 0.00 \\
\hline 35 & 97.81 & 97.81 & 97.96 & 98.08 & 98.18 & 98.26 & 98.33 \\
\hline 36 & 74.69 & 74.69 & 84.66 & 90.38 & $\begin{array}{l}90.10 \\
93.69\end{array}$ & 95.67 & 96.90 \\
\hline 37 & 99.98 & 99.98 & 99.99 & 99.99 & 100.00 & 100.00 & 100.00 \\
\hline 38 & 99.99 & 99.99 & 100.00 & 100.00 & 100.00 & 100.00 & 100.00 \\
\hline 39 & 100.00 & 100.00 & 100.00 & 100.00 & 100.00 & 100.00 & 100.00 \\
\hline 40 & 77.80 & 77.80 & 74.39 & 71.09 & 67.95 & 65.01 & 62.29 \\
\hline 41 & 100.00 & $\begin{array}{l}100.00 \\
\end{array}$ & $\begin{array}{r}100.00 \\
\end{array}$ & 100.00 & 100.00 & 100.00 & 100.00 \\
\hline 42 & 100.00 & 100.00 & 100.00 & 100.00 & 100.00 & 100.00 & 100.00 \\
\hline 43 & 100.00 & 100.00 & 100.00 & 100.00 & 100.00 & 100.00 & 100.00 \\
\hline 44 & 99.96 & 99.96 & 99.99 & 99.99 & 100.00 & 100.00 & 100.00 \\
\hline 45 & 86.39 & 86.39 & 82.62 & 79.18 & 76.08 & 73.32 & 70.85 \\
\hline 46 & 99.82 & 99.82 & 99.73 & 99.63 & 99.52 & 99.40 & 99.28 \\
\hline 47 & 100.00 & 100.00 & 100.00 & 100.00 & 100.00 & 100.00 & 100.00 \\
\hline 48 & 98.66 & 98.66 & 96.82 & 93.26 & 87.18 & 78.07 & 66.27 \\
\hline
\end{tabular}


Table S8. RMS $\Delta$-DP4+ values computed by correlating the GIAO NMR chemical shifts computed at the PCM/mPW1PW91/6-31+G**//B3LYP/6-31G* level of theory (M3P), with $15[\mu, \sigma, v]_{0}$ parameters estimated at the M3P level and allowing the remaining one to oscillate between 6 levels.

\begin{tabular}{|c|c|c|c|c|c|c|c|c|c|c|c|c|c|c|c|c|}
\hline $\begin{array}{c}\text { Comp } \\
\mathbf{N}^{o}\end{array}$ & $\begin{array}{c}{ }^{13} \mathrm{C} \\
\mu_{\mathrm{u}, \mathrm{sp} 2}\end{array}$ & $\begin{array}{c}{ }^{13} \mathrm{C} \\
\mu_{\mathrm{u}, \mathrm{sp} 3}\end{array}$ & $\begin{array}{c}{ }^{1} \mathbf{H} \\
\mu_{\mathrm{u}, \mathrm{sp} 2}\end{array}$ & $\begin{array}{c}{ }^{1} \mathbf{H} \\
\mu_{\mathrm{u}, \mathrm{sp} 3}\end{array}$ & $\begin{array}{c}{ }^{13} \mathrm{C} \\
\sigma_{\mathrm{u}, \mathrm{sp} 2}\end{array}$ & $\begin{array}{c}{ }^{13} \mathrm{C} \\
\sigma_{\mathrm{u}, \mathrm{sp} 3}\end{array}$ & $\begin{array}{c}{ }^{1} \mathbf{H} \\
\sigma_{\mathrm{u}, \mathrm{sp} 2}\end{array}$ & $\begin{array}{c}{ }^{1} \mathbf{H} \\
\sigma_{\mathrm{u}, \mathrm{sp} 3}\end{array}$ & ${ }^{13} \mathrm{C} \sigma_{\mathrm{s}}$ & ${ }^{1} H \sigma_{s}$ & $\begin{array}{c}{ }^{13} \mathrm{C} \\
v_{\mathrm{u}, \mathrm{sp} 2}\end{array}$ & $\begin{array}{c}{ }^{13} \mathrm{C} \\
v_{\mathrm{u}, \mathrm{sp} 3}\end{array}$ & $\begin{array}{c}{ }^{1} \mathrm{H} \\
\mathbf{v}_{\mathrm{u}, \mathrm{sp} 2}\end{array}$ & $\begin{array}{c}{ }^{1} \mathrm{H} \\
\mathbf{v}_{\mathrm{u}, \mathrm{sp} 3}\end{array}$ & $\begin{array}{c}{ }^{13} \mathrm{C} \\
v_{\mathrm{s}}\end{array}$ & ${ }^{1} \mathbf{H} \mathbf{v}_{\mathrm{s}}$ \\
\hline 1 & 0.000 & 0.099 & 0.000 & 0.000 & 0.000 & 0.000 & 0.000 & 0.000 & 0.002 & 0.003 & 0.000 & 0.000 & 0.000 & 0.000 & 0.000 & 0.000 \\
\hline 2 & 0.493 & 33.424 & 11.113 & 0.514 & 0.650 & 15.003 & 2.124 & 0.910 & 2.732 & 0.866 & 0.084 & 3.865 & 0.215 & 0.318 & 0.327 & 2.016 \\
\hline 3 & 0.000 & 1.750 & 0.000 & 0.000 & 0.000 & 0.000 & 0.000 & 0.000 & 0.002 & 0.000 & 0.000 & 0.000 & 0.000 & 0.000 & 0.000 & 0.000 \\
\hline 4 & 2.810 & 40.293 & 0.169 & 7.667 & 0.309 & 26.181 & 0.166 & 0.239 & 0.337 & 0.238 & 0.098 & 1.609 & 0.077 & 0.090 & 0.096 & 0.261 \\
\hline 5 & 0.109 & 11.307 & 0.000 & 20.668 & 0.034 & 4.674 & 0.000 & 5.839 & 1.805 & 0.993 & 0.021 & 2.315 & 0.000 & 0.319 & 0.266 & 0.149 \\
\hline 6 & 0.002 & 0.181 & 0.000 & 0.293 & 0.000 & 0.005 & 0.000 & 0.003 & 0.061 & 0.003 & 0.000 & 0.003 & 0.000 & 0.001 & 0.003 & 0.001 \\
\hline 7 & 3.922 & 17.592 & 0.000 & 5.821 & 0.517 & 1.261 & 0.000 & 2.378 & 0.038 & 6.415 & 0.173 & 1.127 & 0.000 & 0.526 & 0.046 & 0.498 \\
\hline 8 & 0.001 & 0.181 & 0.000 & 0.020 & 0.000 & 0.026 & 0.000 & 0.001 & 0.117 & 0.004 & 0.000 & 0.006 & 0.000 & 0.001 & 0.004 & 0.001 \\
\hline 9 & 0.000 & 0.069 & 0.000 & 0.000 & 0.000 & 0.002 & 0.000 & 0.000 & 0.000 & 0.006 & 0.000 & 0.000 & 0.000 & 0.000 & 0.000 & 0.000 \\
\hline 10 & 0.000 & 0.000 & 0.000 & 0.000 & 0.000 & 0.000 & 0.000 & 0.000 & 0.000 & 0.002 & 0.000 & 0.000 & 0.000 & 0.000 & 0.000 & 0.000 \\
\hline 11 & 0.015 & 0.347 & 0.000 & 0.000 & 0.000 & 0.000 & 0.000 & 0.000 & 0.039 & 0.000 & 0.000 & 0.000 & 0.000 & 0.000 & 0.000 & 0.000 \\
\hline 12 & 0.000 & 0.000 & 0.000 & 0.000 & 0.000 & 0.000 & 0.000 & 0.000 & 0.000 & 0.000 & 0.000 & 0.000 & 0.000 & 0.000 & 0.000 & 0.000 \\
\hline 13 & 1.827 & 3.902 & 0.071 & 36.680 & 0.011 & 0.053 & 0.005 & 5.085 & 0.151 & 0.329 & 0.098 & 0.018 & 0.001 & 0.052 & 0.009 & 0.040 \\
\hline 14 & 0.858 & 39.418 & 1.498 & 1.681 & 0.011 & 2.697 & 0.160 & 0.246 & 1.275 & 0.041 & 0.026 & 0.343 & 0.025 & 0.317 & 0.168 & 0.025 \\
\hline 15 & 22.170 & 35.401 & 1.217 & 37.948 & 6.064 & 3.577 & 0.934 & 4.385 & 0.497 & 1.432 & 1.205 & 0.733 & 0.384 & 0.137 & 0.838 & 1.173 \\
\hline 16 & 0.001 & 0.000 & 0.002 & 0.004 & 0.000 & 0.000 & 0.000 & 0.001 & 0.000 & 0.183 & 0.000 & 0.000 & 0.000 & 0.000 & 0.000 & 0.000 \\
\hline 17 & 0.000 & 0.000 & 0.000 & 0.006 & 0.000 & 0.000 & 0.000 & 0.000 & 0.000 & 0.000 & 0.000 & 0.000 & 0.000 & 0.000 & 0.000 & 0.000 \\
\hline 18 & 7.688 & 41.849 & 0.000 & 18.657 & 0.146 & 14.639 & 0.000 & 8.133 & 3.883 & 10.228 & 1.965 & 4.416 & 0.000 & 0.731 & 4.228 & 1.916 \\
\hline 19 & 3.119 & 8.994 & 0.000 & 26.224 & 0.658 & 2.647 & 0.000 & 5.244 & 3.043 & 1.099 & 0.176 & 0.279 & 0.000 & 3.509 & 0.450 & 3.534 \\
\hline 20 & 4.385 & 17.880 & 0.000 & 21.691 & 0.923 & 6.770 & 0.000 & 0.175 & 4.662 & 3.437 & 0.163 & 2.025 & 0.000 & 1.014 & 1.242 & 0.470 \\
\hline 21 & 16.538 & 35.715 & 0.000 & 30.270 & 4.786 & 6.704 & 0.000 & 3.247 & 17.199 & 1.469 & 2.655 & 2.223 & 0.000 & 0.087 & 1.619 & 0.189 \\
\hline 22 & 15.443 & 51.566 & 0.000 & 8.599 & 3.620 & 2.547 & 0.000 & 1.561 & 7.172 & 0.902 & 1.188 & 0.148 & 0.000 & 0.098 & 1.025 & 0.278 \\
\hline 23 & 5.282 & 39.580 & 0.000 & 16.428 & 0.211 & 4.960 & 0.000 & 2.626 & 17.144 & 4.763 & 1.431 & 0.967 & 0.000 & 3.736 & 1.409 & 6.267 \\
\hline 24 & 4.966 & 45.914 & 0.000 & 15.266 & 0.152 & 5.555 & 0.000 & 3.310 & 17.640 & 5.459 & 1.162 & 0.394 & 0.000 & 0.685 & 0.577 & 3.332 \\
\hline 25 & 0.000 & 0.002 & 0.000 & 0.000 & 0.000 & 0.000 & 0.000 & 0.000 & 0.000 & 0.000 & 0.000 & 0.000 & 0.000 & 0.000 & 0.000 & 0.000 \\
\hline 26 & 0.000 & 18.212 & 0.000 & 0.040 & 0.000 & 0.407 & 0.000 & 0.014 & 0.004 & 0.002 & 0.000 & 0.020 & 0.000 & 0.002 & 0.001 & 0.002 \\
\hline 27 & 0.000 & 48.356 & 0.000 & 0.713 & 0.000 & 16.014 & 0.000 & 1.412 & 4.965 & 1.197 & 0.000 & 4.560 & 0.000 & 34.037 & 0.892 & 0.112 \\
\hline 28 & 0.000 & 0.495 & 0.006 & 0.001 & 0.000 & 0.031 & 0.001 & 0.000 & 0.006 & 0.002 & 0.000 & 0.002 & 0.000 & 0.000 & 0.001 & 0.000 \\
\hline 29 & 0.000 & 0.341 & 0.000 & 0.053 & 0.000 & 0.000 & 0.000 & 0.000 & 0.000 & 0.000 & 0.000 & 0.000 & 0.000 & 0.000 & 0.000 & 0.000 \\
\hline 30 & 0.000 & 0.018 & 0.000 & 37.409 & 0.000 & 0.000 & 0.000 & 0.044 & 0.000 & 0.003 & 0.000 & 0.000 & 0.000 & 0.000 & 0.000 & 0.000 \\
\hline 31 & 0.000 & 1.022 & 0.000 & 5.047 & 0.000 & 0.000 & 0.000 & 0.010 & 0.000 & 0.057 & 0.000 & 0.000 & 0.000 & 0.000 & 0.000 & 0.000 \\
\hline 32 & 0.274 & 27.020 & 2.121 & 0.697 & 0.153 & 3.045 & 0.440 & 0.289 & 1.019 & 0.678 & 0.108 & 0.439 & 0.204 & 0.029 & 0.008 & 0.143 \\
\hline 33 & 7.128 & 30.448 & 0.724 & 20.925 & 0.696 & 0.334 & 0.616 & 2.638 & 8.975 & 0.596 & 0.274 & 0.117 & 1.268 & 0.313 & 0.854 & 1.160 \\
\hline 34 & 1.115 & 0.088 & 4.032 & 0.277 & 0.076 & 0.459 & 1.235 & 0.030 & 0.179 & 3.715 & 0.026 & 0.331 & 0.247 & 0.087 & 0.140 & 0.130 \\
\hline 35 & 1.482 & 34.714 & 1.325 & 29.359 & 0.412 & 2.386 & 0.387 & 21.585 & 2.950 & 0.148 & 0.094 & 0.427 & 0.322 & 0.753 & 0.442 & 0.191 \\
\hline 36 & 7.639 & 16.151 & 10.184 & 37.322 & 2.050 & 0.783 & 10.343 & 21.028 & 8.081 & 13.817 & 0.866 & 0.027 & 20.549 & 8.932 & 1.015 & 8.410 \\
\hline 37 & 0.004 & 0.016 & 0.037 & 0.112 & 0.001 & 0.000 & 0.018 & 0.053 & 0.009 & 0.028 & 0.001 & 0.000 & 0.011 & 0.004 & 0.001 & 0.005 \\
\hline 38 & 0.008 & 0.001 & 0.003 & 4.252 & 0.002 & 0.001 & 0.002 & 0.143 & 0.001 & 0.129 & 0.001 & 0.000 & 0.002 & 0.003 & 0.000 & 0.003 \\
\hline 39 & 0.000 & 0.000 & 0.000 & 0.026 & 0.000 & 0.000 & 0.000 & 0.002 & 0.000 & 0.003 & 0.000 & 0.000 & 0.000 & 0.000 & 0.000 & 0.000 \\
\hline 40 & 12.412 & 6.885 & 39.918 & 25.648 & 1.798 & 3.818 & 7.883 & 16.671 & 8.787 & 2.359 & 0.164 & 0.449 & 5.470 & 3.762 & 1.736 & 5.824 \\
\hline 41 & 0.000 & 0.000 & 0.000 & 0.003 & 0.000 & 0.000 & 0.000 & 0.000 & 0.000 & 0.000 & 0.000 & 0.000 & 0.000 & 0.000 & 0.000 & 0.000 \\
\hline 42 & 0.005 & 0.001 & 0.001 & 2.432 & 0.001 & 0.000 & 0.001 & 0.110 & 0.000 & 0.045 & 0.000 & 0.000 & 0.001 & 0.001 & 0.000 & 0.001 \\
\hline 43 & 0.000 & 0.000 & 0.001 & 0.101 & 0.000 & 0.000 & 0.000 & 0.013 & 0.000 & 0.012 & 0.000 & 0.000 & 0.000 & 0.000 & 0.000 & 0.000 \\
\hline 44 & 0.020 & 0.161 & 0.000 & 0.411 & 0.007 & 0.002 & 0.000 & 0.006 & 0.001 & 4.039 & 0.004 & 0.003 & 0.000 & 0.013 & 0.003 & 0.014 \\
\hline 45 & 21.902 & 32.039 & 0.000 & 33.973 & 3.794 & 0.981 & 0.000 & 17.729 & 0.346 & 4.043 & 1.057 & 0.345 & 0.000 & 1.082 & 0.491 & 5.828 \\
\hline 46 & 0.000 & 44.171 & 0.000 & 7.114 & 0.000 & 0.341 & 0.000 & 0.074 & 1.573 & 0.044 & 0.000 & 0.095 & 0.000 & 0.112 & 0.134 & 0.203 \\
\hline 47 & 0.000 & 0.020 & 0.000 & 0.015 & 0.000 & 0.000 & 0.000 & 0.000 & 0.000 & 0.001 & 0.000 & 0.000 & 0.000 & 0.000 & 0.000 & 0.000 \\
\hline 48 & 0.153 & 6.320 & 0.000 & 0.923 & 0.046 & 9.505 & 0.000 & 0.545 & 12.167 & 0.464 & 0.048 & 2.181 & 0.000 & 3.091 & 1.858 & 12.494 \\
\hline Av. & 3.0 & 14.4 & 1.5 & 9.5 & 0.6 & 2.8 & 0.5 & 2.6 & 2.6 & 1.4 & 0.3 & 0.6 & 0.6 & 1.3 & 0.4 & 1.1 \\
\hline
\end{tabular}


Table S9. Changes in the DP4+ values calculated for the test set (Figure 2) when correlating the GIAO NMR chemical shifts computed at the $\mathrm{PCM} / \mathrm{mPW} 1 \mathrm{PW} 91 / 6-31+\mathrm{G}^{* *} / / \mathrm{B} 3 \mathrm{LYP} / 6-31 \mathrm{G}^{*}$ level of theory (M3P), with randomly generated $[\mu, \sigma, v]$ parameters using the ranges shown in Table S5, showing only the first 40 iterations for each compound. The results obtained with the matched M3P parameters are shown in grey.

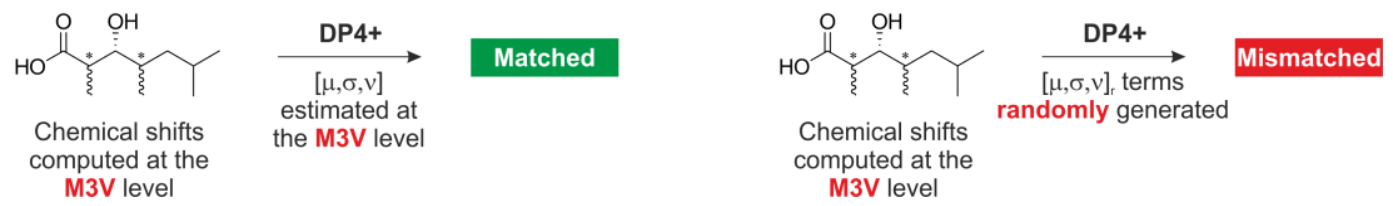

\begin{tabular}{|c|c|c|c|c|c|c|c|c|c|c|c|c|c|c|c|c|c|c|c|c|c|}
\hline \multirow[b]{2}{*}{ Comp $\mathrm{N}^{\circ}$} & \multirow{2}{*}{ M3P } & \multicolumn{20}{|c|}{ Iteration $\mathbf{N}^{\circ}$} \\
\hline & & 1 & 2 & 3 & 4 & 5 & 6 & 7 & 8 & 9 & 10 & 11 & 12 & 13 & 14 & 15 & 16 & 17 & 18 & 19 & 20 \\
\hline 1 & 100.00 & 19.28 & 99.99 & 96.92 & 99.42 & 100.00 & 85.87 & 99.85 & 36.21 & 99.82 & 99.62 & 99.91 & 100.00 & 63.65 & 100.00 & 99.08 & 74.25 & 100.00 & 99.94 & 100.00 & 100.00 \\
\hline 2 & 97.27 & 99.99 & 76.56 & 98.41 & 98.62 & 51.85 & 98.75 & 96.61 & 99.92 & 98.91 & 89.84 & 99.98 & 90.23 & 99.56 & 91.97 & 99.94 & 99.05 & 30.14 & 68.40 & 72.87 & 57.28 \\
\hline 3 & 100.00 & 4.64 & 99.96 & 96.16 & 53.11 & 100.00 & 80.69 & 97.10 & 1.83 & 100.00 & 99.86 & 99.84 & 100.00 & 22.86 & 100.00 & 100.00 & 85.79 & 100.00 & 97.21 & 99.97 & 100.00 \\
\hline 4 & 99.38 & 100.00 & 98.43 & 99.68 & 99.98 & 1.73 & 95.42 & 99.38 & 99.99 & 62.85 & 79.90 & 100.00 & 65.86 & 99.86 & 99.93 & 8.06 & 71.56 & 35.91 & 88.17 & 60.05 & 84.93 \\
\hline 5 & 93.40 & 84.77 & 27.95 & 26.69 & 70.24 & 13.36 & 37.52 & 83.35 & 92.78 & 41.22 & 30.24 & 83.84 & 77.20 & 51.35 & 33.61 & 78.84 & 90.46 & 83.18 & 84.37 & 59.79 & 38.69 \\
\hline 6 & 100.00 & 8.76 & 87.87 & 1.72 & 99.02 & 99.73 & 83.74 & 99.37 & 17.28 & 98.35 & 75.42 & 92.18 & 100.00 & 4.74 & 99.44 & 99.98 & 97.69 & 100.00 & 99.66 & 99.73 & 99.57 \\
\hline 7 & 98.31 & 1.59 & 98.84 & 90.67 & 65.36 & 99.77 & 73.55 & 60.80 & 1.49 & 99.23 & 94.26 & 36.87 & 99.20 & 35.37 & 99.47 & 6.42 & 0.86 & 31.23 & 22.01 & 96.84 & 99.29 \\
\hline 8 & 100.00 & 93.31 & 85.08 & 77.24 & 99.93 & 92.48 & 96.33 & 99.24 & 77.22 & 88.85 & 82.82 & 99.90 & 99.85 & 95.02 & 99.83 & 99.88 & 61.80 & 99.94 & 92.99 & 99.25 & 94.03 \\
\hline 9 & 100.00 & 99.99 & 100.00 & 100.00 & 100.00 & 100.00 & 99.98 & 99.06 & 99.95 & 99.98 & 99.98 & 100.00 & 98.40 & 100.00 & 100.00 & 98.14 & 45.92 & 99.99 & 99.94 & 100.00 & 100.00 \\
\hline 10 & 100.00 & 99.99 & 100.00 & 99.97 & 100.00 & 100.00 & 100.00 & 100.00 & 100.00 & 100.00 & 100.00 & 100.00 & 100.00 & 100.00 & 100.00 & 100.00 & 99.94 & 100.00 & 100.00 & 100.00 & 100.00 \\
\hline 11 & 100.00 & 81.93 & 100.00 & 72.95 & 100.00 & 100.00 & 93.47 & 93.94 & 2.08 & 99.98 & 96.77 & 99.10 & 100.00 & 86.37 & 100.00 & 100.00 & 92.95 & 100.00 & 99.98 & 0.34 & 100.00 \\
\hline 12 & 100.00 & 40.94 & 99.87 & 59.04 & 99.97 & 99.99 & 96.66 & 99.99 & 99.81 & 99.94 & 98.18 & 98.38 & 100.00 & 62.63 & 100.00 & 99.03 & 97.48 & 100.00 & 99.96 & 100.00 & 100.00 \\
\hline 13 & 99.86 & 68.23 & 48.25 & 70.29 & 85.17 & 69.79 & 40.53 & 99.84 & 0.45 & 86.83 & 57.39 & 91.48 & 99.92 & 71.94 & 16.22 & 90.67 & 32.63 & 98.43 & 89.43 & 9.20 & 84.76 \\
\hline 14 & 99.69 & 57.27 & 70.09 & 0.33 & 99.90 & 91.68 & 96.79 & 31.10 & 97.81 & 94.87 & 81.77 & 99.10 & 58.26 & $\begin{array}{l}4.03 \\
\end{array}$ & $\begin{array}{l}100.00 \\
100\end{array}$ & 95.86 & 77.33 & 51.32 & $\begin{array}{l}70.43 \\
70.92\end{array}$ & $\begin{array}{l}6.20 \\
63.59\end{array}$ & 95.28 \\
\hline 15 & 96.16 & 99.58 & 99.89 & 99.97 & 99.96 & 91.24 & 98.73 & 93.40 & 96.94 & 72.59 & 95.20 & 99.59 & 81.24 & 99.89 & 99.97 & 0.07 & 0.12 & 0.07 & 7.14 & 1.23 & 98.73 \\
\hline 16 & 100.00 & 30.38 & 55.60 & 9.62 & 99.63 & 99.31 & 79.22 & 51.50 & 97.77 & 90.26 & 83.16 & 99.57 & 99.58 & 0.87 & 100.00 & 96.37 & 99.34 & 100.00 & 99.84 & 100.00 & 99.44 \\
\hline 17 & 100.00 & 53.38 & 99.93 & 58.96 & 99.37 & 99.88 & 96.85 & 100.00 & 88.74 & 99.92 & 99.29 & 99.99 & 100.00 & 4.69 & 99.99 & 99.73 & 98.97 & 100.00 & 99.99 & 100.00 & 99.98 \\
\hline 18 & 36.13 & 0.02 & 4.49 & 0.98 & 0.13 & 13.28 & 0.76 & 1.75 & 0.10 & 18.06 & 11.86 & 0.15 & 49.07 & 0.14 & 4.98 & 6.60 & 6.12 & 77.21 & 12.64 & 43.89 & 13.60 \\
\hline 19 & 19.85 & 30.12 & 5.21 & 15.32 & 1.88 & 1.45 & 23.02 & 62.54 & 34.81 & 19.43 & 21.95 & 7.91 & 22.51 & 19.07 & 1.87 & 87.76 & 85.20 & 70.96 & 61.94 & 46.96 & 6.89 \\
\hline 20 & 49.27 & 58.53 & 49.60 & 78.87 & 83.74 & 25.49 & 55.86 & 16.34 & 40.42 & 42.54 & 56.53 & 86.25 & 37.12 & 54.50 & 86.76 & 11.20 & 11.20 & 12.64 & 21.10 & 18.64 & 46.40 \\
\hline 21 & 80.31 & 0.93 & 91.99 & 27.27 & 70.23 & 88.77 & 32.92 & 4.82 & 1.52 & 77.18 & 64.99 & 2.78 & 87.79 & 6.80 & 97.40 & 7.83 & 2.60 & 15.22 & 13.51 & 9.25 & 89.55 \\
\hline 22 & 92.78 & 0.01 & 98.08 & 15.87 & 8.04 & 99.48 & 5.11 & 0.80 & 0.02 & 96.32 & 72.49 & 1.05 & 98.52 & 0.38 & 99.46 & 9.30 & 1.81 & 64.96 & 6.60 & 7.91 & 98.47 \\
\hline 23 & 73.30 & 2.22 & 60.74 & 18.20 & $\begin{array}{l}3.09 \\
3.09\end{array}$ & 57.92 & 15.29 & 29.05 & 2.43 & 68.70 & 58.95 & 5.80 & 90.86 & 4.63 & 56.41 & 82.50 & 41.46 & 89.45 & 36.89 & 18.60 & 65.01 \\
\hline 24 & 25.95 & 97.40 & 28.94 & 80.33 & 93.81 & 7.05 & 81.44 & 63.14 & 88.91 & 32.65 & 38.82 & 91.29 & 9.94 & 95.11 & 50.27 & 14.69 & 26.66 & 7.81 & 54.69 & 72.90 & 25.46 \\
\hline 25 & 0.00 & 0.00 & 0.03 & 0.01 & 0.00 & 0.02 & 0.00 & 0.00 & 0.00 & 0.03 & 0.11 & 0.00 & 0.00 & 0.00 & 0.00 & 0.00 & 0.08 & 0.00 & 0.02 & 0.00 & 0.03 \\
\hline 26 & 99.99 & 100.00 & 99.98 & 100.00 & 100.00 & 94.90 & 99.99 & 100.00 & 100.00 & 99.41 & 99.62 & 100.00 & 99.84 & 100.00 & 100.00 & 43.80 & 25.19 & 4.78 & 48.84 & 98.55 & 99.28 \\
\hline 27 & 98.23 & 0.59 & 93.57 & 19.15 & 0.01 & 99.81 & 0.25 & 0.22 & 0.03 & 98.94 & 88.88 & 97.99 & 91.20 & 0.63 & 99.81 & 99.93 & 94.91 & 100.00 & 96.28 & 9.25 & 99.39 \\
\hline 28 & 100.00 & 100.00 & 96.22 & 99.82 & 99.53 & 96.14 & 99.77 & $\begin{array}{l}0.22 \\
99.91\end{array}$ & 99.99 & 99.92 & $\begin{array}{l}0.00 \\
99.10\end{array}$ & 100.00 & 99.68 & 99.90 & 99.98 & 100.00 & $\begin{array}{l}99.84 \\
99.81\end{array}$ & 99.45 & $\begin{array}{l}90.12 \\
99.12\end{array}$ & 99.91 & 98.27 \\
\hline 29 & 100.00 & 58.27 & 100.00 & 100.00 & 100.00 & 100.00 & 98.77 & 95.22 & 14.18 & 100.00 & 100.00 & 100.00 & 100.00 & 99.33 & 100.00 & 0.12 & 0.00 & 80.97 & 9.84 & 99.98 & 100.00 \\
\hline 30 & 100.00 & 100.00 & 100.00 & 100.00 & 100.00 & 100.00 & 100.00 & 100.00 & 100.00 & 100.00 & 100.00 & 100.00 & 100.00 & 100.00 & 100.00 & 0.42 & 0.45 & 7.87 & 99.18 & 100.00 & 100.00 \\
\hline 31 & 100.00 & 0.60 & 99.26 & 1.95 & 88.27 & 99.97 & 4.57 & 99.26 & 16.33 & 99.95 & 93.04 & 91.65 & 100.00 & 0.62 & 100.00 & 97.88 & 89.13 & 100.00 & 99.85 & 100.00 & 99.98 \\
\hline 32 & 99.30 & 14.88 & 93.92 & 86.39 & 89.97 & 82.24 & 70.99 & 76.76 & 5.99 & 93.08 & 92.98 & 99.61 & 62.66 & 29.11 & 99.99 & 71.48 & 63.37 & 73.07 & 90.66 & 87.88 & 88.50 \\
\hline 33 & 97.79 & $\begin{array}{l}14.60 \\
1.60\end{array}$ & 87.63 & 17.92 & 3.47 & 95.16 & 16.87 & 97.07 & 0.54 & 76.13 & 56.41 & 15.48 & 99.72 & 3.55 & 0.97 & 98.17 & 71.69 & 98.72 & 53.48 & 38.06 & 88.66 \\
\hline 34 & 0.34 & 0.30 & 9.86 & 13.10 & 21.44 & 0.63 & 1.43 & 0.04 & 0.08 & 1.62 & 6.61 & 8.75 & 5.96 & 0.37 & 0.01 & 0.62 & 1.93 & 0.02 & 0.00 & 0.00 & 1.04 \\
\hline 35 & 97.81 & 99.40 & 31.07 & 94.65 & 95.67 & 5.86 & 91.89 & 96.82 & 99.82 & 61.11 & 57.52 & 99.16 & 90.10 & 97.15 & 57.58 & 6.51 & 35.25 & 0.29 & 36.75 & 97.52 & 40.78 \\
\hline 36 & 74.69 & 3.07 & 95.25 & 0.03 & 38.54 & 37.12 & 6.10 & 99.96 & 58.79 & 24.02 & 24.57 & 17.39 & 97.27 & 5.21 & 69.37 & 10.18 & 58.19 & 100.00 & 98.98 & 99.98 & 86.04 \\
\hline 37 & 99.98 & 99.79 & 97.53 & 0.74 & 99.43 & 99.38 & 99.49 & 100.00 & 99.88 & 99.18 & 96.63 & 99.68 & 99.78 & 98.44 & 99.99 & 99.12 & 91.04 & 99.88 & 99.96 & 99.91 & 97.84 \\
\hline 38 & 99.99 & 66.14 & 94.81 & $\begin{array}{l}0.74 \\
27.49\end{array}$ & 98.92 & 96.64 & 72.29 & 99.99 & 99.79 & 88.85 & 75.77 & 93.51 & 99.98 & 55.83 & 99.96 & 90.92 & 97.65 & $\begin{array}{l}100.00 \\
100.00\end{array}$ & 99.95 & 100.00 & 98.94 \\
\hline 39 & 100.00 & 97.19 & 92.12 & 95.49 & 99.21 & 99.33 & 98.31 & 99.96 & 99.90 & 99.40 & 96.24 & 99.88 & 100.00 & 96.00 & 99.98 & 96.44 & 86.74 & 99.35 & 96.99 & 100.00 & 99.28 \\
\hline 40 & 77.80 & 38.09 & 98.41 & 6.76 & 87.58 & 3.01 & 7.69 & 99.88 & 9.01 & 1.15 & 24.72 & 4.34 & 17.03 & 54.71 & 95.16 & 0.02 & 0.55 & 91.65 & 91.70 & 4.07 & 68.25 \\
\hline 41 & 100.00 & 99.88 & 98.26 & 32.59 & 99.94 & 99.99 & 99.83 & 100.00 & 100.00 & 99.98 & 99.29 & 99.98 & 100.00 & 99.20 & 100.00 & 100.00 & 99.95 & 100.00 & 100.00 & 100.00 & 99.93 \\
\hline 42 & 100.00 & 65.52 & 90.37 & 23.41 & 99.46 & 93.07 & 70.81 & 99.97 & 99.75 & 77.18 & 67.37 & 90.35 & 99.98 & 52.09 & 99.88 & 76.28 & 94.36 & 100.00 & 99.83 & 100.00 & 98.39 \\
\hline $\begin{array}{l}42 \\
43\end{array}$ & 100.00 & $\begin{array}{l}94.02 \\
94.88\end{array}$ & 90.20 & 95.18 & 97.49 & 98.70 & 96.66 & 99.41 & 99.42 & 99.18 & 95.18 & 99.70 & 99.98 & 94.49 & 99.92 & $\begin{array}{l}1.28 \\
93.68\end{array}$ & $\begin{array}{l}794.50 \\
72.66\end{array}$ & 95.25 & 92.12 & 99.98 & 97.78 \\
\hline 44 & 99.96 & 99.86 & 71.72 & 42.83 & 99.86 & 93.88 & 99.67 & 99.99 & 99.97 & 98.18 & 87.14 & 99.97 & 98.71 & 95.85 & 99.95 & 100.00 & 99.99 & 100.00 & 100.00 & 100.00 & 99.07 \\
\hline 45 & 86.39 & 9.21 & 93.56 & 76.94 & 97.14 & 88.97 & 44.02 & 3.14 & 8.08 & 53.89 & $\begin{array}{l}68.02 \\
68.02\end{array}$ & 28.10 & 92.05 & 32.54 & 98.82 & 0.33 & 0.58 & 1.59 & 1.52 & 0.64 & 83.08 \\
\hline 46 & 99.82 & 4.47 & 99.99 & 99.97 & 92.27 & 100.00 & 85.11 & 5.39 & 0.05 & 99.85 & 99.83 & 99.55 & 99.96 & 77.00 & 100.00 & 10.75 & 0.02 & 6.45 & 0.27 & 0.23 & 99.92 \\
\hline 47 & 100.00 & 58.43 & 99.74 & 54.99 & 99.91 & 99.99 & 94.87 & 99.97 & 97.71 & 99.96 & 98.41 & 99.91 & 100.00 & 38.73 & 100.00 & 99.98 & 99.54 & 100.00 & 99.98 & 100.00 & 99.99 \\
\hline 48 & 98.66 & $\begin{array}{l}30.43 \\
99.74\end{array}$ & 98.51 & 99.93 & 97.74 & 97.60 & $\begin{array}{l}90.81 \\
90.62\end{array}$ & 86.73 & 88.86 & 89.72 & $\begin{array}{l}95.41 \\
95.47\end{array}$ & 99.83 & 93.76 & 99.46 & $\begin{array}{l}48.20 \\
\end{array}$ & 95.83 & 36.54 & 8.48 & 7.92 & 0.36 & 83.59 \\
\hline
\end{tabular}

\begin{tabular}{|c|c|c|c|c|c|c|c|c|c|c|c|c|c|c|c|c|c|c|c|c|c|}
\hline \multirow[b]{2}{*}{ Comp $\mathrm{N}^{\circ}$} & \multirow{2}{*}{ M3P } & \multicolumn{20}{|c|}{ Iteration $\mathbf{N}^{\circ}$} \\
\hline & & 21 & 22 & 23 & 24 & 25 & 26 & 27 & 28 & 29 & 30 & 31 & 32 & 33 & 34 & 35 & 36 & 37 & 38 & 39 & 40 \\
\hline 1 & 100.00 & 99.98 & 99.37 & 99.73 & 99.98 & 88.90 & 98.47 & 97.03 & 99.78 & 100.00 & 99.54 & 90.68 & 98.98 & 98.27 & 99.98 & 99.99 & 100.00 & 100.00 & 99.98 & 99.74 & 100.00 \\
\hline 2 & 97.27 & 99.92 & 99.50 & 99.65 & 66.43 & 98.86 & 100.00 & 99.94 & 99.55 & 95.34 & 88.60 & 98.60 & 99.83 & 93.96 & 97.92 & 76.79 & 14.47 & 42.60 & 94.57 & 99.98 & 84.18 \\
\hline 3 & 100.00 & 99.88 & 98.63 & 100.00 & 97.69 & 98.60 & 99.63 & 99.12 & 99.98 & 99.94 & 99.95 & 96.47 & 99.94 & 50.28 & 97.41 & 99.99 & 100.00 & 100.00 & 100.00 & 99.93 & 100.00 \\
\hline 4 & 99.38 & 99.88 & 71.62 & 0.18 & 99.78 & 39.51 & 99.91 & 98.63 & 58.62 & 99.90 & 53.15 & 89.81 & 88.92 & 99.19 & 99.40 & 77.06 & 0.06 & 0.72 & 2.82 & 99.95 & 1.24 \\
\hline 5 & 93.40 & 93.93 & 66.48 & 89.77 & 43.57 & 86.34 & 93.37 & 77.30 & 75.06 & 70.54 & 44.87 & 82.49 & 85.04 & 59.77 & 67.41 & 34.92 & 14.24 & 16.65 & 73.07 & 89.46 & 16.79 \\
\hline 6 & 100.00 & 99.50 & 96.22 & 100.00 & 64.57 & 97.77 & 95.66 & 96.86 & 99.93 & 94.16 & 98.74 & 97.96 & 99.73 & 24.76 & 84.95 & 96.46 & 100.00 & 98.27 & 99.99 & 99.56 & 99.97 \\
\hline 7 & 98.31 & 43.52 & 2.06 & 17.97 & 84.86 & 10.87 & 0.01 & 21.75 & 21.63 & 94.21 & 97.86 & 82.11 & 23.22 & 81.20 & 87.17 & 97.99 & 99.90 & 99.98 & 82.66 & 9.35 & 99.62 \\
\hline 8 & 100.00 & 98.71 & 92.81 & 98.06 & 98.41 & 69.88 & 50.56 & 98.10 & 99.83 & 99.80 & 75.21 & 93.84 & 98.66 & 96.17 & 99.79 & 89.30 & 82.00 & 66.09 & 99.56 & 99.93 & 99.14 \\
\hline 9 & 100.00 & 100.00 & 99.36 & 86.35 & 100.00 & 98.97 & 98.90 & 99.89 & 99.95 & 100.00 & 99.25 & 99.93 & 99.99 & 100.00 & 100.00 & 99.98 & 99.58 & 100.00 & 99.95 & 100.00 & 100.00 \\
\hline 10 & 100.00 & 100.00 & 100.00 & 100.00 & 100.00 & 100.00 & 100.00 & 100.00 & 100.00 & 100.00 & 99.99 & 100.00 & 100.00 & 100.00 & 100.00 & 100.00 & 100.00 & 100.00 & 100.00 & 100.00 & 100.00 \\
\hline 11 & 100.00 & 35.37 & 63.34 & 98.16 & 99.98 & 3.11 & 19.11 & 1.56 & 100.00 & 99.73 & 99.95 & 0.00 & 99.24 & 55.01 & 97.45 & 99.50 & 100.00 & 97.40 & 99.66 & 99.88 & 100.00 \\
\hline 12 & 100.00 & 99.98 & 99.99 & 99.80 & 99.93 & 95.99 & 99.49 & 99.56 & 99.32 & 100.00 & 98.08 & 99.99 & 94.73 & 99.55 & 99.99 & 99.67 & 99.97 & 100.00 & 99.92 & 99.45 & 100.00 \\
\hline 13 & 99.86 & 84.81 & 1.75 & 93.13 & 73.63 & 1.92 & 21.05 & 6.09 & 95.40 & 95.17 & 91.68 & 0.06 & 98.14 & 33.41 & 38.89 & 54.18 & 10.75 & 96.12 & 96.85 & 98.91 & 69.24 \\
\hline 14 & 99.69 & 99.97 & 97.57 & 1.38 & 77.91 & 75.91 & 99.37 & 99.41 & 98.45 & 99.83 & 92.38 & 97.73 & 97.75 & 57.57 & 99.61 & 70.65 & 0.05 & 21.17 & 42.19 & 99.78 & 97.73 \\
\hline 15 & 96.16 & 76.68 & 0.13 & 0.00 & 99.82 & 0.09 & 0.00 & 19.17 & 4.53 & 98.14 & 74.88 & 34.72 & 2.28 & 99.16 & 99.45 & 93.26 & 2.05 & 47.05 & 0.05 & 87.71 & 65.01 \\
\hline 16 & 100.00 & 100.00 & 99.99 & 99.02 & 99.57 & 99.52 & 99.85 & 99.90 & 97.98 & 99.99 & 92.93 & 99.98 & 98.77 & 82.14 & 100.00 & 45.75 & 6.25 & 99.72 & 99.43 & 98.65 & 99.63 \\
\hline 17 & 100.00 & 100.00 & 99.99 & 100.00 & 99.89 & 99.95 & 100.00 & 100.00 & 99.87 & 100.00 & 96.15 & 100.00 & 99.98 & 94.34 & 99.97 & 99.96 & 99.99 & 100.00 & 100.00 & 99.98 & 99.98 \\
\hline 18 & 36.13 & 0.12 & 10.55 & 74.69 & 6.20 & 7.31 & 0.08 & 1.44 & 4.80 & 2.32 & 11.72 & 4.77 & 2.09 & 0.78 & 0.84 & 19.93 & 81.94 & 69.10 & 57.06 & 0.20 & 13.61 \\
\hline 19 & 19.85 & 35.20 & 79.96 & 91.72 & 12.59 & 74.07 & 93.05 & 62.96 & 63.76 & 7.96 & 4.52 & 25.83 & 53.01 & 24.02 & 19.80 & 16.40 & 0.21 & 3.07 & 73.43 & 26.94 & 9.28 \\
\hline 20 & 49.27 & 31.77 & 13.88 & 3.73 & 74.13 & $\begin{array}{l}15.99 \\
\end{array}$ & 8.39 & 25.85 & 30.87 & 75.56 & 32.85 & 38.31 & 34.38 & 55.34 & 60.16 & $\begin{array}{r}43.80 \\
\end{array}$ & 0.81 & 34.12 & 16.25 & 60.49 & $\begin{array}{r}42.72 \\
\end{array}$ \\
\hline 21 & 80.31 & 0.16 & 4.98 & 0.41 & 65.94 & 2.31 & 0.00 & 1.15 & 11.71 & 10.92 & 80.84 & 9.06 & 2.28 & 7.06 & 21.56 & 72.17 & 12.88 & 73.53 & 11.80 & 2.14 & 87.84 \\
\hline 22 & 92.78 & 0.01 & 2.80 & 9.98 & 55.35 & 1.09 & 0.00 & 0.29 & 11.67 & 3.52 & 96.08 & 1.33 & 1.81 & 0.39 & 1.06 & 94.10 & 98.66 & 95.81 & 40.16 & 0.85 & 98.83 \\
\hline 23 & 73.30 & 2.02 & 44.15 & 97.78 & 27.07 & 36.57 & 30.84 & 15.05 & 58.42 & 5.44 & 47.45 & 10.92 & 33.56 & 4.21 & 2.50 & 75.46 & 6.46 & 31.90 & 90.49 & 20.03 & 82.77 \\
\hline 24 & 25.95 & 96.88 & 32.87 & 1.72 & 67.46 & 49.94 & 60.62 & 82.38 & 35.05 & 91.43 & 23.77 & 84.60 & 63.89 & 95.50 & 97.42 & 20.27 & 0.01 & 27.22 & 9.72 & 78.58 & 12.20 \\
\hline 25 & 0.00 & $\begin{array}{l}0.00 \\
0.00\end{array}$ & $\begin{array}{l}2.0, \\
0.01\end{array}$ & 0.17 & $\begin{array}{l}0.40 \\
0.01\end{array}$ & 0.05 & $\begin{array}{l}0.02 \\
0.00\end{array}$ & 0.00 & 0.00 & 0.00 & 0.48 & 0.00 & 0.00 & 0.00 & 0.00 & 0.09 & $\begin{array}{l}0.01 \\
0.10\end{array}$ & 0.14 & 0.02 & 0.00 & 0.00 \\
\hline 26 & 99.99 & 100.00 & 97.56 & 0.03 & 99.99 & 51.45 & 100.00 & 99.99 & 96.60 & 100.00 & 96.98 & 99.97 & 99.51 & 99.99 & 99.99 & 99.42 & 24.63 & 97.36 & 10.09 & 100.00 & 96.05 \\
\hline 27 & 98.23 & 51.75 & 29.57 & 100.00 & 64.71 & 91.61 & 93.33 & 16.48 & 99.58 & 91.95 & 98.64 & 0.09 & 99.45 & 0.29 & 12.20 & 98.95 & 100.00 & 79.29 & 99.98 & 96.12 & 99.85 \\
\hline 28 & 100.00 & 100.00 & 99.96 & 99.99 & 97.66 & 99.94 & 100.00 & 100.00 & 99.98 & 99.99 & 95.67 & 99.94 & 100.00 & 99.82 & 99.99 & 99.08 & 78.19 & 96.62 & 99.97 & 100.00 & 99.82 \\
\hline 29 & 100.00 & 99.99 & 0.88 & 0.29 & 100.00 & 1.15 & 0.13 & 92.45 & 38.29 & 100.00 & 100.00 & 99.82 & 83.45 & 99.88 & 99.99 & 100.00 & 100.00 & 100.00 & 94.35 & 99.71 & 100.00 \\
\hline 30 & 100.00 & 100.00 & 94.09 & 0.00 & 100.00 & 60.43 & 0.52 & 99.99 & 72.88 & 100.00 & 99.98 & 100.00 & 97.14 & 100.00 & 100.00 & 100.00 & 92.74 & 100.00 & 17.50 & 99.99 & 100.00 \\
\hline 31 & 100.00 & 99.98 & 99.85 & 100.00 & 97.04 & 99.13 & 100.00 & 99.78 & 98.30 & 99.98 & 99.82 & 99.92 & 99.54 & 38.76 & 96.14 & 99.52 & 100.00 & 100.00 & 100.00 & 92.71 & 99.95 \\
\hline 32 & 99.30 & 90.41 & 94.73 & 18.33 & 98.58 & 72.97 & 95.75 & 59.46 & 85.91 & 99.53 & 79.97 & 7.90 & 92.57 & 64.77 & 93.13 & 90.55 & 3.89 & 76.52 & 68.57 & 99.34 & 91.64 \\
\hline 33 & 97.79 & 26.40 & 32.68 & 99.35 & 22.22 & 55.42 & 81.71 & 21.43 & 95.56 & 11.86 & 67.54 & 4.33 & 81.45 & 10.56 & 2.03 & 85.77 & 99.97 & 71.48 & 96.95 & 83.91 & 97.41 \\
\hline 34 & 0.34 & 0.01 & 0.10 & 0.01 & 12.58 & 0.19 & 0.17 & 0.13 & 1.87 & 0.02 & 7.91 & 0.00 & 0.41 & 0.14 & 0.01 & 2.43 & 0.06 & 0.05 & 0.18 & 3.35 & 0.30 \\
\hline 35 & 97.81 & $\begin{array}{l}0.01 \\
99.80\end{array}$ & $\begin{array}{l}0.10 \\
30.56\end{array}$ & $\begin{array}{l}.01 \\
1.59\end{array}$ & $\begin{array}{l}1.58 \\
63.72\end{array}$ & 60.13 & $\begin{array}{l}0.17 \\
89.39\end{array}$ & 99.26 & $\begin{array}{l}1.87 \\
15.45\end{array}$ & $\begin{array}{l}0.02 \\
95.18\end{array}$ & $\begin{array}{l}.191 \\
59.58\end{array}$ & $\begin{array}{l}0.00 \\
99.81\end{array}$ & $\begin{array}{l}0.41 \\
73.22\end{array}$ & $\begin{array}{l}0.14 \\
94.01\end{array}$ & $\begin{array}{l}0.01 \\
96.30\end{array}$ & $\begin{array}{l}2.43 \\
22.06\end{array}$ & $\begin{array}{l}0.00 \\
0.02\end{array}$ & $\begin{array}{l}0.05 \\
48.69\end{array}$ & $\begin{array}{l}0.18 \\
11.06\end{array}$ & 92.01 & $\begin{array}{l}0.30 \\
3.09\end{array}$ \\
\hline 36 & 74.69 & 95.49 & 88.65 & 29.34 & 97.65 & 53.15 & 94.03 & 71.86 & 45.35 & 96.35 & 9.54 & 55.85 & 25.16 & 90.06 & 61.59 & 83.43 & 97.42 & 69.82 & 65.16 & 23.80 & 39.14 \\
\hline 37 & 99.98 & 99.99 & $\begin{array}{l}60.00 \\
98.71\end{array}$ & 99.44 & 95.97 & 98.19 & 99.44 & 99.93 & 97.64 & 99.85 & 97.88 & 99.99 & 99.59 & 99.07 & 99.80 & 98.15 & $\begin{array}{l}80.48 \\
80.48\end{array}$ & 99.62 & $\begin{array}{l}9.10 \\
98.53\end{array}$ & 99.86 & 99.67 \\
\hline 38 & 99.99 & 100.00 & 99.96 & 99.03 & 99.27 & 98.56 & 99.99 & 99.87 & 96.83 & 99.96 & 76.32 & 99.95 & 97.12 & 98.43 & 99.86 & 89.55 & 88.00 & 99.38 & 99.08 & 96.94 & 98.09 \\
\hline 39 & 100.00 & 100.00 & 98.27 & 98.95 & 97.67 & 98.69 & 99.64 & 99.98 & 97.98 & 99.99 & 95.11 & 100.00 & 99.52 & 99.74 & 99.99 & 94.67 & 90.40 & 99.99 & 99.76 & 99.89 & 99.85 \\
\hline 40 & 77.80 & 2.26 & $\begin{array}{l}5.24 \\
0.24\end{array}$ & $\begin{array}{c}9.93 \\
0.00\end{array}$ & 97.04 & $\begin{array}{c}9.09 \\
0.32\end{array}$ & $\begin{array}{c}9.04 \\
0.01\end{array}$ & 0.20 & 0.99 & 65.23 & $\begin{array}{l}1.00 \\
1.11\end{array}$ & 1.10 & 0.20 & 94.50 & 5.24 & 91.33 & $\begin{array}{l}87.40 \\
87.19\end{array}$ & 8.54 & $\begin{array}{l}0.10 \\
0.20\end{array}$ & 27.05 & 1.32 \\
\hline 41 & 100.00 & 100.00 & 100.00 & 100.00 & 98.18 & 99.99 & 100.00 & 100.00 & 99.98 & 100.00 & 99.95 & 100.00 & 100.00 & 98.54 & 99.99 & 99.57 & 97.51 & 99.99 & 100.00 & 100.00 & 100.00 \\
\hline 42 & 100.00 & 99.99 & 99.73 & 91.11 & 98. & 94.66 & 99.8 & 99. & 92.00 & 99.8 & 68.52 & 99. & 91.47 & 97.6 & 99.7 & 82.55 & 77.91 & 98.59 & 96.95 & 96.50 & 96.12 \\
\hline 43 & 100.00 & 100.00 & 92.68 & 98.20 & 93.93 & 97.36 & 96.22 & 99.72 & 96.12 & 99.94 & 95.13 & 99.98 & 99.03 & 99.27 & 99.94 & 93.70 & 90.85 & 99.95 & 99.42 & 99.65 & 99.56 \\
\hline 44 & 99.96 & 100.00 & 100.00 & 100.00 & 96.98 & 100.00 & 100.00 & 99.99 & 99.98 & 100.00 & 95.42 & 100.00 & 100.00 & 99.93 & 100.00 & 91.07 & 44.59 & 99.99 & 100.00 & 99.97 & 99.78 \\
\hline 45 & 86.39 & 0.33 & 0.25 & 0.04 & 85.84 & 0.35 & 0.00 & 0.65 & 1.93 & 16.31 & 82.39 & 10.87 & 0.58 & 12.04 & 8.05 & 67.30 & 94.23 & 71.10 & 1.46 & 4.55 & 57.22 \\
\hline 46 & 99.82 & 3.85 & 0.03 & 0.81 & 99.44 & 0.16 & 0.00 & 0.69 & 54.42 & 96.04 & 99.75 & 1.77 & 17.53 & 19.94 & 27.04 & 99.94 & 100.00 & 99.90 & 63.50 & 92.47 & 100.00 \\
\hline 47 & 100.00 & 100.00 & 100.00 & 100.00 & 99.82 & 99.80 & 100.00 & 99.98 & 99.98 & 100.00 & 99.82 & 99.99 & 99.98 & 90.48 & 99.80 & 99.77 & 100.00 & 100.00 & 100.00 & 99.97 & 100.00 \\
\hline 48 & 98.66 & 75.48 & 16.56 & 5.26 & 87.55 & 15.96 & 78.80 & 80.32 & 95.19 & 79.00 & 73.09 & 7.89 & 82.37 & 94.39 & 87.50 & 96.95 & 99.51 & 20.48 & 49.73 & 99.86 & 94.78 \\
\hline
\end{tabular}


Table S10. Changes in the DP4+ values calculated for the test set (Figure 2) when correlating the GIAO NMR chemical shifts computed at the $\mathrm{PCM} / \mathrm{mPW} 1 \mathrm{PW} 91 / 6-31+\mathrm{G}^{* *} / \mathrm{B} 3 \mathrm{LYP} / 6-31 \mathrm{G}^{*}$ level of theory (M3P), with randomly generated $[\mu, \sigma, v]$ parameters using the ranges shown in Table S6, showing only the first 40 iterations for each compound. The results obtained with the matched M3P parameters are shown in grey.
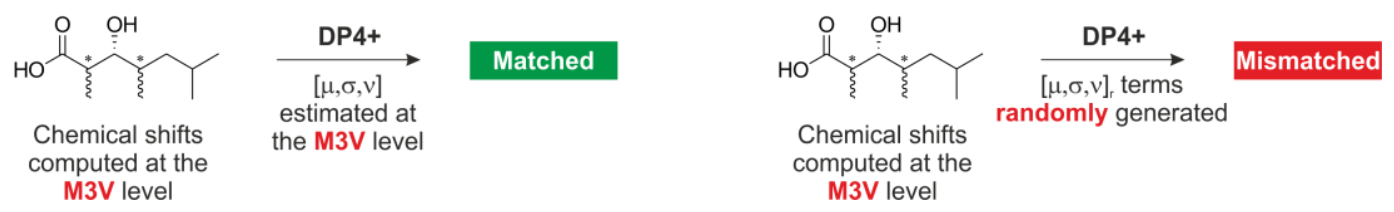

\begin{tabular}{|c|c|c|c|c|c|c|c|c|c|c|c|c|c|c|c|c|c|c|c|c|c|}
\hline \multirow[b]{2}{*}{ Comp $\mathbf{N}^{\circ}$} & \multirow[b]{2}{*}{ M3P } & \multicolumn{20}{|c|}{ Iteration $\mathbf{N}^{\circ}$} \\
\hline & & 1 & 2 & 3 & 4 & 5 & 6 & 7 & 8 & 9 & 10 & 11 & 12 & 13 & 14 & 15 & 16 & 17 & 18 & 19 & 20 \\
\hline 1 & 100.00 & 100.00 & 100.00 & 100.00 & 100.00 & 100.00 & 100.00 & 100.00 & 100.00 & 100.00 & 100.00 & 100.00 & 100.00 & 100.00 & 100.00 & 100.00 & 100.00 & 100.00 & 100.00 & 100.00 & 100.00 \\
\hline 2 & 97.27 & 95.58 & 95.72 & 96.65 & 98.41 & 99.02 & 98.99 & 95.53 & 97.13 & 91.28 & 96.33 & 97.15 & 95.02 & 94.86 & 98.41 & 93.73 & 96.49 & 96.96 & 96.56 & 94.13 & 86.21 \\
\hline 3 & 100.00 & 100.00 & 100.00 & 100.00 & 100.00 & 100.00 & 100.00 & 100.00 & 100.00 & 100.00 & 100.00 & 100.00 & 100.00 & 100.00 & 100.00 & 100.00 & 100.00 & 100.00 & 100.00 & 100.00 & 100.00 \\
\hline 4 & 99.38 & 99.72 & 99.88 & 98.93 & 99.74 & 99.66 & 99.37 & 98.98 & 99.29 & 98.91 & 97.63 & 98.27 & 98.39 & 99.39 & 99.70 & 99.77 & 99.08 & 99.88 & 99.68 & 99.50 & 98.94 \\
\hline 5 & 93.40 & 93.47 & 96.19 & 91.00 & 95.36 & 92.10 & 92.96 & 94.26 & 94.74 & 95.98 & 91.23 & 89.47 & 92.28 & 91.70 & 94.47 & 95.85 & 94.70 & 94.91 & 93.37 & 91.22 & 91.14 \\
\hline 6 & 100.00 & 99.99 & 100.00 & 99.99 & 99.99 & 100.00 & 100.00 & 100.00 & 100.00 & 100.00 & 100.00 & 100.00 & 100.00 & 100.00 & 99.99 & 99.99 & 100.00 & 100.00 & 100.00 & 99.99 & 100.00 \\
\hline 7 & 98.31 & 97.47 & 98.97 & 98.08 & 94.31 & 97.61 & 97.03 & 97.62 & 98.76 & 99.10 & 99.21 & 98.54 & 99.13 & 99.27 & 94.03 & 98.83 & 99.57 & 97.99 & 99.05 & 98.17 & 99.20 \\
\hline 8 & 100.00 & 99.99 & 100.00 & 99.99 & 99.99 & 100.00 & 100.00 & 99.99 & 100.00 & 99.99 & 100.00 & 100.00 & 99.99 & 100.00 & 100.00 & 99.99 & 100.00 & 100.00 & 100.00 & 99.99 & 99.99 \\
\hline 9 & 100.00 & 100.00 & 100.00 & 100.00 & 100.00 & 100.00 & 100.00 & 100.00 & 100.00 & 100.00 & 100.00 & 100.00 & 100.00 & 100.00 & 100.00 & 100.00 & 100.00 & 100.00 & 100.00 & 100.00 & 100.00 \\
\hline 10 & 100.00 & 100.00 & 100.00 & 100.00 & 100.00 & 100.00 & 100.00 & 100.00 & 100.00 & 100.00 & 100.00 & 100.00 & 100.00 & 100.00 & 100.00 & 100.00 & 100.00 & 100.00 & 100.00 & 100.00 & 100.00 \\
\hline 11 & 100.00 & 100.00 & 100.00 & 100.00 & 100.00 & 100.00 & 100.00 & 100.00 & 100.00 & 100.00 & 100.00 & 100.00 & 100.00 & 100.00 & 100.00 & 100.00 & 100.00 & 100.00 & 100.00 & 100.00 & 100.00 \\
\hline $\begin{array}{l}11 \\
12\end{array}$ & 100.00 & 100.00 & $\begin{array}{l}100.00 \\
\end{array}$ & 100.00 & $\begin{array}{l}100.00 \\
\end{array}$ & 100.00 & 100.00 & $\begin{array}{l}100.00 \\
\end{array}$ & $\begin{array}{l}100.00 \\
\end{array}$ & 100.00 & 100.00 & 100.00 & 100.00 & 100.00 & 100.00 & 100.00 & 100.00 & 100.00 & 100.00 & 100.00 & 100.00 \\
\hline 13 & 99.86 & 99.88 & 99.97 & 99.77 & 99.52 & 99.81 & 99.16 & 99.85 & 99.95 & 99.98 & 99.50 & 99.65 & 99.96 & 99.91 & 99.36 & 99.94 & 99.98 & 99.92 & 99.72 & 99.92 & 99.64 \\
\hline 14 & 99.69 & 99.52 & 99.45 & 99.58 & 99.56 & 99.88 & 99.93 & 99.56 & 99.69 & 97.96 & 99.70 & 99.64 & 99.50 & 99.62 & 99.88 & 99.24 & 99.63 & 99.63 & 99.60 & 99.52 & 99.12 \\
\hline 15 & 96.16 & 98.50 & 98.86 & 91.49 & 96.97 & 95.39 & 89.00 & 96.15 & 96.71 & 96.03 & 96.42 & 91.10 & 86.89 & 96.69 & 97.32 & 98.98 & 96.72 & 99.28 & 98.69 & 98.18 & 97.31 \\
\hline 16 & 100.00 & 100.00 & 100.00 & 100.00 & 100.00 & 100.00 & 100.00 & 100.00 & 100.00 & 100.00 & 100.00 & 100.00 & 100.00 & 100.00 & 100.00 & 100.00 & 100.00 & 100.00 & 100.00 & $\begin{array}{l}100.00 \\
\end{array}$ & 100.00 \\
\hline $\begin{array}{l}10 \\
17\end{array}$ & 100.00 & $\begin{array}{l}100.00 \\
\end{array}$ & 100.00 & 100.00 & 100.00 & 100.00 & 100.00 & 100.00 & 100.00 & $\begin{array}{l}100.00 \\
\end{array}$ & 100.00 & 100.00 & 100.00 & 100.00 & 100.00 & 100.00 & 100.00 & 100.00 & 100.00 & 100.00 & 100.00 \\
\hline 18 & 36.13 & 44.31 & 48.39 & 36.25 & 33.73 & 30.48 & 39.66 & 32.62 & 25.77 & 46.95 & 33.49 & 45.20 & 42.83 & 46.02 & 22.55 & 24.54 & 37.81 & 37.62 & 40.27 & 28.05 & 47.97 \\
\hline 19 & 19.85 & 29.38 & 18.94 & 20.98 & 22.89 & 21.28 & 26.62 & 19.02 & 13.12 & 17.69 & 18.02 & 28.39 & 14.86 & 20.13 & 22.80 & 11.38 & 12.55 & 23.20 & 21.58 & 18.82 & 20.70 \\
\hline $\begin{array}{l}19 \\
20\end{array}$ & $\begin{array}{l}19.83 \\
49.27\end{array}$ & $\begin{array}{l}2.38 \\
45.43\end{array}$ & $\begin{array}{l}18.94 \\
50.96\end{array}$ & $\begin{array}{l}20.98 \\
49.26\end{array}$ & $\begin{array}{l}22.89 \\
53.56\end{array}$ & $\begin{array}{l}21.28 \\
49.35\end{array}$ & $\begin{array}{l}26.02 \\
48.17\end{array}$ & $\begin{array}{l}19.02 \\
53.12\end{array}$ & $\begin{array}{l}13.12 \\
53.31\end{array}$ & $\begin{array}{l}1.09 \\
46.16\end{array}$ & $\begin{array}{l}18.02 \\
47.00\end{array}$ & $\begin{array}{l}28.39 \\
39.21\end{array}$ & $\begin{array}{l}14.86 \\
49.42\end{array}$ & $\begin{array}{l}20.13 \\
44.27\end{array}$ & $\begin{array}{l}22.80 \\
55.68\end{array}$ & $\begin{array}{l}11.38 \\
57.55\end{array}$ & $\begin{array}{l}12.33 \\
52.64\end{array}$ & $\begin{array}{l}23.20 \\
48.56\end{array}$ & $\begin{array}{l}21.58 \\
48.16\end{array}$ & $\begin{array}{l}18.82 \\
51.11\end{array}$ & $\begin{array}{l}20.10 \\
45.55\end{array}$ \\
\hline 21 & 80.31 & $\begin{array}{l}79.43 \\
79.03\end{array}$ & 84.78 & $\begin{array}{l}79.40 \\
77.46\end{array}$ & 71.25 & 83.02 & 86.04 & 79.70 & 79.88 & $\begin{array}{l}70.10 \\
70.83\end{array}$ & 85.45 & 82.01 & $\begin{array}{l}99.42 \\
81.75\end{array}$ & 84.51 & $\begin{array}{l}68.50 \\
680\end{array}$ & 77.13 & 82.17 & $\begin{array}{l}80.00 \\
80.90\end{array}$ & $\begin{array}{l}40.10 \\
91.14\end{array}$ & 78.77 & 89.00 \\
\hline 22 & 92.78 & 93.89 & 95.44 & 89.69 & 88.44 & 91.89 & 94.76 & 93.10 & 92.12 & 90.04 & 95.48 & 92.43 & 91.89 & 94.85 & 88.53 & 92.90 & 95.55 & 94.95 & 97.18 & 92.31 & 96.52 \\
\hline 23 & 73.30 & 84.33 & 81.57 & 73.24 & 78.73 & 83.63 & 91.27 & 66.06 & 48.40 & 59.31 & 70.74 & 89.71 & 61.94 & 77.24 & 64.23 & 49.28 & 51.72 & 8096 & 91.49 & 63.46 & 86,07 \\
\hline 24 & $\begin{array}{l}73.30 \\
25.95\end{array}$ & $\begin{array}{l}4.435 \\
18.40\end{array}$ & $\begin{array}{l}21.71 \\
21.79\end{array}$ & $\begin{array}{l}5.24 \\
26.30\end{array}$ & 24.10 & $\begin{array}{l}8.03 \\
15.82\end{array}$ & $\begin{array}{l}91.27 \\
11.46\end{array}$ & $\begin{array}{l}00.00 \\
34.33\end{array}$ & $\begin{array}{l}48.40 \\
44.30\end{array}$ & 39.68 & $\begin{array}{l}0.14 \\
25.05\end{array}$ & $\begin{array}{l}10.65 \\
105\end{array}$ & $\begin{array}{l}\begin{array}{l}31.94 \\
35.15\end{array} \\
\end{array}$ & $\begin{array}{r}17.24 \\
21.40\end{array}$ & $\begin{array}{l}04.25 \\
35.88\end{array}$ & $\begin{array}{l}49.28 \\
43.76\end{array}$ & $\begin{array}{l}11.62 \\
44.60\end{array}$ & $\begin{array}{l}80.90 \\
20.17\end{array}$ & $\begin{array}{l}91.49 \\
10.15\end{array}$ & $\begin{array}{l}0.40 \\
31.44\end{array}$ & $\begin{array}{l}80.07 \\
14.59\end{array}$ \\
\hline 25 & 0.00 & 0.00 & 0.00 & 0.00 & 0.00 & 0.00 & 0.00 & 0.00 & 0.00 & 0.00 & 0.00 & & 0.00 & 0.00 & & & & & & & 0.00 \\
\hline 26 & 99.99 & 99.99 & 100.00 & 99.99 & 100.00 & 100.00 & 99.99 & $\begin{array}{l}0.00 \\
99.99\end{array}$ & 100.00 & 99.99 & 100.00 & 99.99 & 99.98 & 99.99 & 100.00 & 100.00 & 99.99 & 100.00 & 100.00 & 99.99 & 99.98 \\
\hline 27 & 98.23 & 98.80 & 96.32 & 97.67 & 99.38 & 98.44 & 99.87 & 97.28 & 92.41 & 93.50 & 97.86 & 97.89 & 93.96 & 97.84 & 99.60 & 87.11 & 94.20 & 98.87 & 97.74 & 93.70 & 98.93 \\
\hline 28 & $\begin{array}{l}100.00 \\
\end{array}$ & $\begin{array}{l}10.000 \\
1000\end{array}$ & $\begin{array}{l}300.02 \\
100.00\end{array}$ & 100.00 & 100.00 & $\begin{array}{l}100.00 \\
1000\end{array}$ & 100.00 & 100.00 & $\begin{array}{l}100.41 \\
10.00\end{array}$ & 100.00 & $\begin{array}{l}100.00 \\
\end{array}$ & 100.00 & 100.00 & 100.00 & 100.00 & 100.00 & 100.00 & $\begin{array}{l}300.07 \\
100.00\end{array}$ & 100.00 & 100.00 & 100.00 \\
\hline $\begin{array}{l}28 \\
29\end{array}$ & 100.00 & $\begin{array}{l}100.00 \\
100.00\end{array}$ & 100.00 & 100.00 & 100.00 & 100.00 & 100.00 & $\begin{array}{l}100.00 \\
1000\end{array}$ & 100.00 & 100. & 100. & 100.00 & 100.00 & 100.00 & 100.00 & 100.00 & & 100.00 & 100. & 100.00 & 100.00 \\
\hline 30 & 100.00 & 99.99 & 100.00 & 100.00 & 99.99 & 100.00 & 99.99 & 100.00 & 100.00 & 100.00 & 100 & 100.00 & 100.00 & 100.00 & 99. & 100.00 & 100 & 100.00 & 100 & 100.00 & 100.00 \\
\hline 31 & 100.00 & 100.00 & 100.00 & 100.00 & 100.00 & 100.00 & 100.00 & 100.00 & 100.00 & 100.00 & 100.00 & 100.00 & 100.00 & 100.00 & 100.00 & 100.00 & 100.00 & 100.00 & 100.00 & 100.00 & 100.00 \\
\hline 32 & 99.30 & 98.40 & 98.78 & 99.27 & 99.24 & 99.56 & 99.71 & 99.33 & 99.39 & 99.00 & 99.31 & 99.30 & 99.42 & 99.16 & 99.66 & 99.09 & 99.10 & 98.65 & 98.53 & 98.51 & 98.70 \\
\hline 33 & 97.79 & 97.67 & 98.62 & 96.73 & 98.05 & 98.17 & 98.38 & 97. & 98.21 & 97. & 98. & 98.30 & 93.89 & 97.81 & 95.70 & 98.29 & 97.4 & 98.57 & 99.65 & 96.93 & 98.11 \\
\hline $\begin{array}{l}33 \\
34\end{array}$ & 0.34 & 1.13 & $\begin{array}{l}98.62 \\
1.08\end{array}$ & 0.48 & $\begin{array}{c}98.05 \\
0.78\end{array}$ & $\begin{array}{l}98.17 \\
1.04\end{array}$ & $\begin{array}{l}98.38 \\
2.32\end{array}$ & 0.2 & $\begin{array}{c}98.21 \\
0.14\end{array}$ & 0.0 & $\begin{array}{c}98 . \\
0.0\end{array}$ & $\begin{array}{c}98.30 \\
0.62\end{array}$ & $\begin{array}{c}93.89 \\
0.10\end{array}$ & 0.15 & $\begin{array}{l}95.10 \\
0.25\end{array}$ & $\begin{array}{c}98.29 \\
0.11\end{array}$ & 0.0 & $\begin{array}{l}98.57 \\
0.67\end{array}$ & $\begin{array}{l}99.63 \\
1.44\end{array}$ & $\begin{array}{l}90.93 \\
0.44\end{array}$ & $\begin{array}{c}98.11 \\
0.10\end{array}$ \\
\hline 35 & 97.81 & $\begin{array}{l}1.11 \\
97.79\end{array}$ & 99.43 & $\begin{array}{l}0.40 \\
96.18\end{array}$ & 98.75 & 96.06 & 86.02 & $\begin{array}{l}97.21 \\
97.55\end{array}$ & 98.9 & 99. & 96.78 & $\begin{array}{l}0.02 \\
92.90\end{array}$ & $\begin{array}{l}0.10 \\
96.91\end{array}$ & 97. & 96.39 & 99. & 99. & 98. & $\begin{array}{l}1.4 \\
98 .\end{array}$ & 97.6 & 95.66 \\
\hline 36 & 74.69 & 59.97 & 82.62 & 77.0 & 57.96 & 55.81 & 42.1 & 50.90 & 84. & 93. & 82. & 74. & 80. & 87. & 33. & 88. & 86 & 75. & 83.93 & 82.50 & 84.31 \\
\hline 37 & 99. & 99.9 & 99.9 & 99. & 99.98 & 99.98 & 99.97 & 99.99 & 99. & 100.00 & 99.99 & 99. & 99.98 & 99.99 & 99.99 & 99.99 & 99. & 99. & 99.9 & 99.96 & 99.99 \\
\hline 38 & 99.99 & 99.98 & $\begin{array}{l}9.90 .00 \\
100.00\end{array}$ & 99.98 & 99.96 & 99.98 & 99.95 & 99.98 & 100.00 & 100.00 & 100.00 & 99.99 & $\begin{array}{l}100.00 \\
100\end{array}$ & $\begin{array}{l}100.09 \\
100\end{array}$ & 99.95 & $\begin{array}{l}100.09 \\
100\end{array}$ & 100.00 & 99.99 & 99.99 & 99.99 & 100.00 \\
\hline $\begin{array}{l}38 \\
39\end{array}$ & $\begin{array}{r}9.99 \\
100.00\end{array}$ & $\begin{array}{l}9.98 \\
100.00\end{array}$ & $\begin{array}{l}100000 \\
100.00\end{array}$ & $\begin{array}{c}9.98 \\
100.00\end{array}$ & $\begin{array}{c}99.96 \\
100.00\end{array}$ & 100.08 & $\begin{array}{l}9.95 \\
100.00\end{array}$ & $\begin{array}{c}9.98 .98 \\
10000\end{array}$ & $\begin{array}{l}100000 \\
100.00\end{array}$ & $\begin{array}{l}100000 \\
100.00\end{array}$ & $\begin{array}{l}100000 \\
100.00\end{array}$ & $\begin{array}{c}9 \\
100,00\end{array}$ & $\begin{array}{l}100.00 \\
100.00\end{array}$ & $\begin{array}{l}100000 \\
100.00\end{array}$ & $\begin{array}{l}9.95 \\
100.00\end{array}$ & $\begin{array}{l}100000 \\
100.00\end{array}$ & $\begin{array}{l}100000 \\
100.00\end{array}$ & $\begin{array}{c}9.99 \\
100.00\end{array}$ & $\begin{array}{c}9.99 .90 \\
100.00\end{array}$ & $\begin{array}{c}9.99 \\
100.00\end{array}$ & $\begin{array}{l}100000 \\
100.00\end{array}$ \\
\hline 40 & 77. & 82 . & 89. & 76. & 82. & 75. & 72. & & & & & & 73. & 75 & 82 & & & & & & 88.06 \\
\hline 41 & & 100 & 100 & 100 & 100 & 100 & 100 & & $\begin{array}{l}07 \\
100\end{array}$ & & & & 100 & 100 & 100 & 100 & & 100. & & $\begin{array}{l}100.00 \\
\end{array}$ & $\begin{array}{l}0.00 \\
100.00\end{array}$ \\
\hline 42 & 100.00 & 99.99 & 100.00 & 99.99 & 99.99 & 99.99 & 99.98 & 99.99 & 100.00 & 100.00 & 100.00 & 99.99 & 100.00 & 100.00 & 99.98 & 100.00 & 100.00 & 100.00 & 100.00 & 100.00 & 100.00 \\
\hline $\begin{array}{l}42 \\
43\end{array}$ & $\begin{array}{l}100.00 \\
\end{array}$ & 100.00 & 100.00 & 100.00 & 100.00 & 100.00 & 100.00 & 100.00 & 100.00 & 100.00 & 100.00 & 100.00 & 100.00 & 100.00 & 100.00 & 100.00 & 100.00 & 100.00 & 100.00 & 100.00 & 100.00 \\
\hline 44 & 99.96 & 99.74 & 99.87 & 99.96 & 99.88 & 99.91 & 99.91 & 99.93 & 99.99 & 99.99 & 99.99 & 99.95 & 99.98 & 99.98 & 99.94 & 99.97 & 99.99 & 99.85 & 99.86 & 99.94 & 99.92 \\
\hline 45 & 86.39 & 90.57 & 96.64 & 82.14 & 86.07 & & 90.31 & 91.92 & 88. & 83.8 & 73. & 81. & 88 & 84. & 80 & 93. & 86. & 91. & 95. & 85.03 & 90.36 \\
\hline 46 & 99.82 & 99.86 & 99.91 & 99.73 & 99.8 & 99.5 & 99. & 99. & $\begin{array}{l}0.0 \\
99 .\end{array}$ & 99. & 99. & $\begin{array}{l}9.1 . \\
99 .\end{array}$ & 99.5 & 99. & 99. & 99.58 & $\begin{array}{l}0.0 .5 \\
99.5\end{array}$ & 99.87 & 99.97 & 99.44 & 99.82 \\
\hline 47 & 100.00 & 100.00 & 100.00 & 100.00 & 100.00 & 100.00 & 100.00 & 100.00 & 100.00 & 10000 & 100.00 & 100.00 & 100,00 & 100.00 & 100.00 & 100.00 & 100.00 & 100.00 & 100.00 & 100.00 & 100.00 \\
\hline 48 & 98.66 & 99.40 & 98.96 & 98.13 & 99.40 & 99.47 & 99.79 & 98.36 & 97.06 & 94.38 & 98.61 & 99.31 & 90.28 & 97.73 & 99.68 & 94.95 & 93.34 & 99.44 & 99.63 & 97.84 & 96.93 \\
\hline
\end{tabular}

\begin{tabular}{|c|c|c|c|c|c|c|c|c|c|c|c|c|c|c|c|c|c|c|c|c|c|}
\hline \multirow[b]{2}{*}{ Comp $\mathrm{N}^{\circ}$} & \multirow{2}{*}{ M3P } & \multicolumn{20}{|c|}{ Iteration $\mathbf{N}^{\circ}$} \\
\hline & & 21 & 22 & 23 & 24 & 25 & 26 & 27 & 28 & 29 & 30 & 31 & 32 & 33 & 34 & 35 & 36 & 37 & 38 & 39 & 40 \\
\hline 1 & 100.00 & 100.00 & 100.00 & 100.00 & 100.00 & 100.00 & 100.00 & 100.00 & 100.00 & 100.00 & 100.00 & 100.00 & 100.00 & 100.00 & 100.00 & 100.00 & 100.00 & 100.00 & 100.00 & 100.00 & 100.00 \\
\hline 2 & 97.27 & 97.82 & 98.05 & 99.46 & 97.09 & 97.11 & 96.20 & 98.48 & 98.66 & 98.16 & 95.05 & 98.17 & 96.66 & 98.41 & 97.29 & 95.05 & 92.92 & 98.91 & 96.48 & 97.95 & 96.74 \\
\hline 3 & 100.00 & 100.00 & 100.00 & 100.00 & 100.00 & 100.00 & 100.00 & 100.00 & 100.00 & 100.00 & 100.00 & 100.00 & 100.00 & 100.00 & 100.00 & 100.00 & 100.00 & 100.00 & 100.00 & 100.00 & 100.00 \\
\hline 4 & 99.38 & 98.44 & 99.54 & 99.77 & 99.05 & 99.53 & 99.15 & 99.85 & 99.28 & 99.49 & 99.28 & 99.59 & 95.36 & 99.83 & 99.35 & 99.68 & 97.33 & 98.87 & 97.04 & 98.49 & 99.49 \\
\hline 5 & 93.40 & 90.87 & 92.07 & 94.07 & 93.27 & 95.71 & 92.84 & 96.57 & 93.18 & 92.40 & 94.72 & 93.46 & 92.40 & 95.66 & 92.36 & 95.39 & 92.53 & 91.60 & 90.56 & 94.32 & 92.21 \\
\hline 6 & 100.00 & 100.00 & 100.00 & 99.99 & 100.00 & 100.00 & 100.00 & 100.00 & 100.00 & 100.00 & 100.00 & 100.00 & 99.99 & 100.00 & 100.00 & 99.99 & 100.00 & 99.99 & 100.00 & 100.00 & 100.00 \\
\hline 7 & 98.31 & 99.11 & 97.06 & 89.84 & 98.65 & 97.64 & 99.32 & 96.27 & 95.75 & 96.42 & 98.55 & 97.59 & 98.33 & 97.97 & 97.27 & 98.69 & 99.31 & 93.90 & 98.97 & 97.93 & 97.33 \\
\hline 8 & 100.00 & 100.00 & 100.00 & 100.00 & 99.99 & 100.00 & 100.00 & 100.00 & 99.99 & 100.00 & 99.99 & 100.00 & 99.99 & 100.00 & 100.00 & 99.99 & 99.99 & 99.99 & 100.00 & 100.00 & 99.99 \\
\hline 9 & 100.00 & 100.00 & 100.00 & 100.00 & 100.00 & 100.00 & 100.00 & 100.00 & 100.00 & 100.00 & 100.00 & 100.00 & 100.00 & 100.00 & 100.00 & 100.00 & 100.00 & 100.00 & 100.00 & 100.00 & 100.00 \\
\hline 10 & 100.00 & 100.00 & 100.00 & 100.00 & 100.00 & 100.00 & 100.00 & 100.00 & 100.00 & 100.00 & 100.00 & 100.00 & 100.00 & 100.00 & 100.00 & 100.00 & 100.00 & 100.00 & 100.00 & 100.00 & 100.00 \\
\hline 11 & 100.00 & 100.00 & 100.00 & 100.00 & 100.00 & 100.00 & 100.00 & 100.00 & 100.00 & 100.00 & 100.00 & 100.00 & 100.00 & 100.00 & 100.00 & 100.00 & 100.00 & 100.00 & 100.00 & 100.00 & 100.00 \\
\hline 12 & 100.00 & 100.00 & 100.00 & 100.00 & 100.00 & 100.00 & 100.00 & 100.00 & 100.00 & 100.00 & 100.00 & 100.00 & 100.00 & 100.00 & 100.00 & 100.00 & 100.00 & 100.00 & 100.00 & 100.00 & 100.00 \\
\hline 13 & 99.86 & 99.86 & 99.06 & 99.43 & 99.91 & 99.91 & 99.95 & 99.94 & 99.47 & 99.71 & 99.90 & 99.92 & 99.59 & 99.93 & 99.34 & 99.92 & 99.89 & 99.29 & 99.41 & 99.75 & 99.27 \\
\hline 14 & 99.69 & 99.87 & 99.92 & 99.90 & 99.64 & 99.63 & 99.78 & 99.77 & 99.76 & 99.91 & 99.61 & 99.82 & 99.53 & 99.71 & 99.56 & 99.38 & 98.36 & 99.62 & 99.78 & 99.71 & 99.76 \\
\hline 15 & 96.16 & 85.33 & 95.42 & 96.35 & 93.60 & 94.13 & 92.49 & 98.39 & 96.69 & 97.53 & 98.66 & 94.45 & 80.88 & 98.00 & 93.01 & 98.64 & 93.41 & 94.24 & 95.58 & 90.73 & 95.09 \\
\hline 16 & 100.00 & 100.00 & 100.00 & 100.00 & 100.00 & 100.00 & 100.00 & 100.00 & 100.00 & 100.00 & 100.00 & 100.00 & 100.00 & 100.00 & 100.00 & 100.00 & 100.00 & 100.00 & 100.00 & 100.00 & 100.00 \\
\hline 17 & 100.00 & 100.00 & 100.00 & 100.00 & 100.00 & 100.00 & 100.00 & 100.00 & 100.00 & 100.00 & 100.00 & 100.00 & 100.00 & 100.00 & 100.00 & 100.00 & 100.00 & 100.00 & 100.00 & 100.00 & 100.00 \\
\hline 18 & 36.13 & 42.14 & 27.46 & 20.11 & 26.94 & 40.44 & 39.03 & 19.49 & 26.87 & 24.02 & 38.10 & 17.73 & 52.97 & 24.95 & 36.10 & 30.17 & 51.85 & 20.98 & 41.81 & 40.55 & 28.87 \\
\hline 19 & 19.85 & 18.53 & 19.92 & 28.88 & 15.82 & 18.09 & 15.23 & 16.20 & 23.15 & 23.05 & 18.32 & 12.95 & 25.31 & 14.64 & 21.60 & 14.72 & 19.06 & 24.21 & 22.85 & 20.38 & 18.92 \\
\hline 20 & 49.27 & 45.93 & 52.46 & 50.95 & 50.02 & 52.92 & 48.22 & 56.49 & 52.05 & 52.33 & 53.59 & 56.69 & 48.07 & 53.33 & 50.10 & 54.53 & 44.88 & 50.64 & 46.58 & 51.51 & 51.74 \\
\hline 21 & 80.31 & 85.74 & 84.41 & 61.20 & 62.86 & 76.64 & 83.12 & 74.87 & 76.46 & 75.44 & 83.32 & 65.94 & 78.70 & 80.07 & 85.57 & 73.49 & 84.00 & 66.00 & 89.07 & 85.66 & 73.25 \\
\hline 22 & 92.78 & 93.71 & 94.31 & 84.47 & 83.97 & 90.60 & 93.16 & 90.83 & 91.42 & 91.07 & 95.58 & 84.25 & 92.40 & 92.81 & 93.23 & 92.07 & 93.55 & 83.50 & 96.09 & 93.87 & 89.00 \\
\hline 23 & 73.30 & 79.18 & 76.62 & 76.12 & 50.19 & 71.60 & 68.92 & 66.47 & 75.15 & 67.73 & 67.46 & 44.90 & 81.07 & 69.68 & 84.41 & 58.74 & 78.08 & 68.74 & 84.56 & 80.52 & 73.26 \\
\hline 24 & 25.95 & 20.17 & 22.42 & 24.23 & 41.52 & 31.36 & 28.24 & 31.49 & 24.69 & 30.08 & 34.23 & 45.70 & 24.38 & 26.45 & 16.96 & 37.28 & 22.22 & 27.24 & 16.84 & 23.15 & 23.95 \\
\hline 25 & 0.00 & 0.00 & 0.00 & 0.00 & 0.00 & 0.00 & 0.00 & 0.00 & 0.00 & 0.00 & 0.00 & 0.00 & 0.00 & 0.00 & 0.00 & 0.00 & 0.00 & 0.00 & 0.00 & 0.00 & 0.00 \\
\hline 26 & 99.99 & 99.99 & 99.99 & 100.00 & 100.00 & 99.99 & 99.99 & 100.00 & 99.99 & 99.99 & 99.99 & 100.00 & 99.94 & 100.00 & 99.99 & 100.00 & 99.99 & 100.00 & 99.98 & 99.99 & 99.99 \\
\hline 27 & 98.23 & 99.32 & 99.55 & 99.76 & 96.91 & 99.14 & 97.34 & 95.85 & 99.10 & 99.48 & 98.98 & 94.87 & 99.26 & 94.08 & 97.92 & 94.27 & 95.64 & 96.93 & 99.55 & 99.20 & 99.21 \\
\hline 28 & 100.00 & 100.00 & 100.00 & 100.00 & 100.00 & 100.00 & 100.00 & 100.00 & 100.00 & 100.00 & 100.00 & 100.00 & 100.00 & 100.00 & 100.00 & 100.00 & 100.00 & 100.00 & 100.00 & 100.00 & 100.00 \\
\hline 29 & 100.00 & 100.00 & 100.00 & 100.00 & 100.00 & 100.00 & 100.00 & 100.00 & 100.00 & 100.00 & 100.00 & 100.00 & 100.00 & 100.00 & 100.00 & 100.00 & 100.00 & 100.00 & 100.00 & 100.00 & 100.00 \\
\hline 30 & 100.00 & 100.00 & 100.00 & 99.97 & 100.00 & 100.00 & 100.00 & 100.00 & 99.99 & 99.99 & 100.00 & 100.00 & 99.99 & 100.00 & 100.00 & 100.00 & 100.00 & 99.99 & 100.00 & 100.00 & 100.00 \\
\hline 31 & 100.00 & 100.00 & 100.00 & 99.99 & 100.00 & 100.00 & 100.00 & 100.00 & 100.00 & 99.99 & 100.00 & 100.00 & 100.00 & 100.00 & 100.00 & 100.00 & 100.00 & 99.99 & 100.00 & 100.00 & 100.00 \\
\hline 32 & 99.30 & 99.65 & 99.64 & 99.53 & 99.59 & 99.55 & 99.64 & 99.59 & 99.13 & 99.46 & 98.37 & 99.64 & 99.24 & 99.56 & 99.38 & 99.17 & 98.51 & 99.33 & 98.72 & 99.44 & 99.63 \\
\hline 33 & 97.79 & 97.29 & 98.06 & 97.25 & 94.99 & 96.39 & 97.52 & 99.06 & 98.16 & 96.91 & 98.17 & 96.00 & 95.37 & 98.73 & 98.50 & 97.16 & 98.41 & 97.17 & 98.85 & 98.50 & 96.60 \\
\hline 34 & 0.34 & 0.21 & 0.63 & 2.30 & 0.03 & 0.29 & 0.13 & 2.71 & 0.71 & 0.24 & 0.10 & 0.25 & 0.20 & 0.62 & 1.33 & 0.07 & 0.09 & 0.46 & 0.22 & 0.40 & 0.22 \\
\hline 35 & 97.81 & 91.15 & 92.40 & 95.84 & 98.65 & 98.21 & 97.69 & 99.12 & 97.14 & 94.37 & 98.55 & 98.28 & 93.31 & 99.22 & 95.18 & 99.50 & 98.38 & 96.48 & 92.13 & 96.22 & 96.18 \\
\hline 36 & 74.69 & 83.36 & 52.13 & 47.89 & 88.58 & 68.20 & 87.43 & 72.66 & 54.34 & 41.23 & 63.5 & 82.24 & 43.75 & 79.87 & 60.04 & 79.86 & 90.42 & 47.65 & 70.93 & 50.22 & 72.75 \\
\hline 37 & 99.98 & 99.98 & 99.97 & 99.95 & 99.99 & 99.99 & 99.99 & 99.99 & 99.97 & 99.98 & 99.9 & 99.98 & 99.97 & 99.99 & 99.97 & 99.99 & 99.99 & 99. & 99. & 99.99 & 99.98 \\
\hline 38 & 99.99 & 100.00 & 99.96 & 99.93 & 100.00 & 99.99 & 100.00 & 99.99 & 99.96 & 99.97 & 99.99 & 99.99 & 99.96 & 99.99 & 99.95 & 100.00 & 100.00 & 99.94 & 99.99 & 99.97 & 99.99 \\
\hline 39 & 100.00 & 100.00 & 100.00 & 100.00 & 100.00 & 100.00 & 100.00 & 100.00 & 100.00 & 100.00 & 100.00 & 100.00 & 100.00 & 100.00 & 100.00 & 100.00 & 100.00 & 100.00 & 100.00 & 100.00 & 100.00 \\
\hline 40 & 77.80 & 63.41 & 62.23 & 64.41 & 68.34 & 85.01 & 66.36 & 83.31 & 71.52 & 63.28 & 71.71 & 54.94 & 79.74 & 85.28 & 86.79 & 85.77 & 86.43 & 79.26 & 66.01 & 78.94 & 77.59 \\
\hline 41 & 100.00 & 100.00 & 100.00 & 100.00 & 100.00 & 100.00 & 100.00 & 100.00 & 100.00 & 100.00 & 100.00 & 100.00 & 100.00 & 100.00 & 100.00 & 100.00 & 100.00 & 100.00 & 100.00 & 100.00 & 100.00 \\
\hline 42 & 100.00 & 100.00 & 99. & 99. & 100 & 100. & 100. & 100 & 99.98 & 99 & 100 & 100 & 99. & 100 & 99.99 & 100.00 & 100.00 & 99.97 & 100.00 & 99.99 & 99.99 \\
\hline 43 & 100.00 & 100.00 & 100.00 & 100.00 & 100.00 & 100.00 & 100.00 & 100.00 & 100.00 & 100.00 & 100.00 & 100.00 & 100.00 & 100.00 & 100.00 & 100.00 & 100.00 & 100.00 & 100.00 & 100.00 & 100.00 \\
\hline 44 & 99.96 & 99.99 & 99.95 & 99.92 & 100.00 & 99.96 & 99.99 & 99.93 & 99.92 & 99.96 & 99.97 & 99.99 & 99.96 & 99.94 & 99.77 & 99.97 & 99.98 & 99.92 & 99.96 & 99.94 & 99.97 \\
\hline 45 & 86.39 & 80.01 & 88.03 & 68.89 & 56.50 & 90.83 & 85.45 & 94.52 & 81.01 & 75.24 & 87.89 & 78.77 & 82.54 & 94.20 & 93.97 & 88.82 & 84.89 & 70.78 & 81.69 & 91.07 & 78.18 \\
\hline 46 & 99.82 & 99.86 & 99.90 & 99.84 & 99.03 & 99.8 & 99.82 & 99.93 & 99.8 & 99.7 & 99. & 99.4 & 99.8 & 99.89 & 99.92 & 99.58 & 99.76 & 99. & 99. & 99.94 & 99.75 \\
\hline 47 & 100.00 & 100.00 & 100.00 & 100.00 & 100.00 & 100.00 & 100.00 & 100.00 & 100.00 & 100.00 & 100.00 & 100.00 & 100.00 & 100.00 & 100.00 & 100.00 & 100.00 & 100.00 & 100.00 & 100.00 & 100.00 \\
\hline 48 & 98.66 & 98.04 & 99.28 & 99.85 & 95.50 & 98.28 & 97.12 & 99.62 & 99.51 & 99.48 & 97.91 & 97.33 & 97.67 & 99.04 & 99.22 & 97.02 & 95.62 & 99.40 & 98.95 & 98.95 & 98.44 \\
\hline
\end{tabular}


Table S11. Overall changes (after 10.000 iterations) in the DP4+ values calculated for the test set (Figure 2) when correlating the GIAO NMR chemical shifts computed at the PCM/mPW1PW91/6-31+G**//B3LYP/6-31G* level of theory (M3P), with randomly generated $[\mu, \sigma, v]$ parameters using the ranges shown in Table S5 (left) and Table S6 (right).
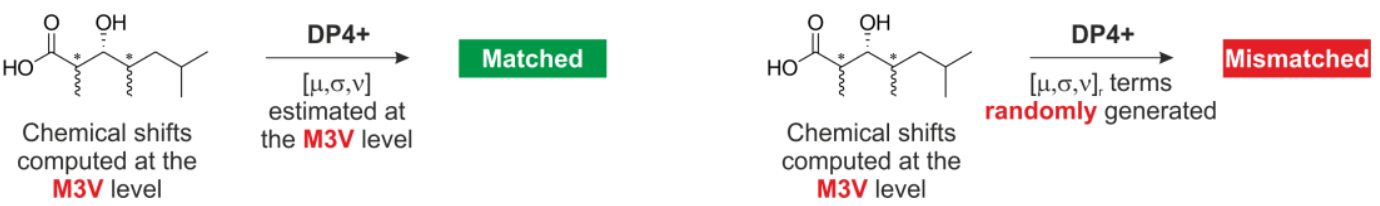

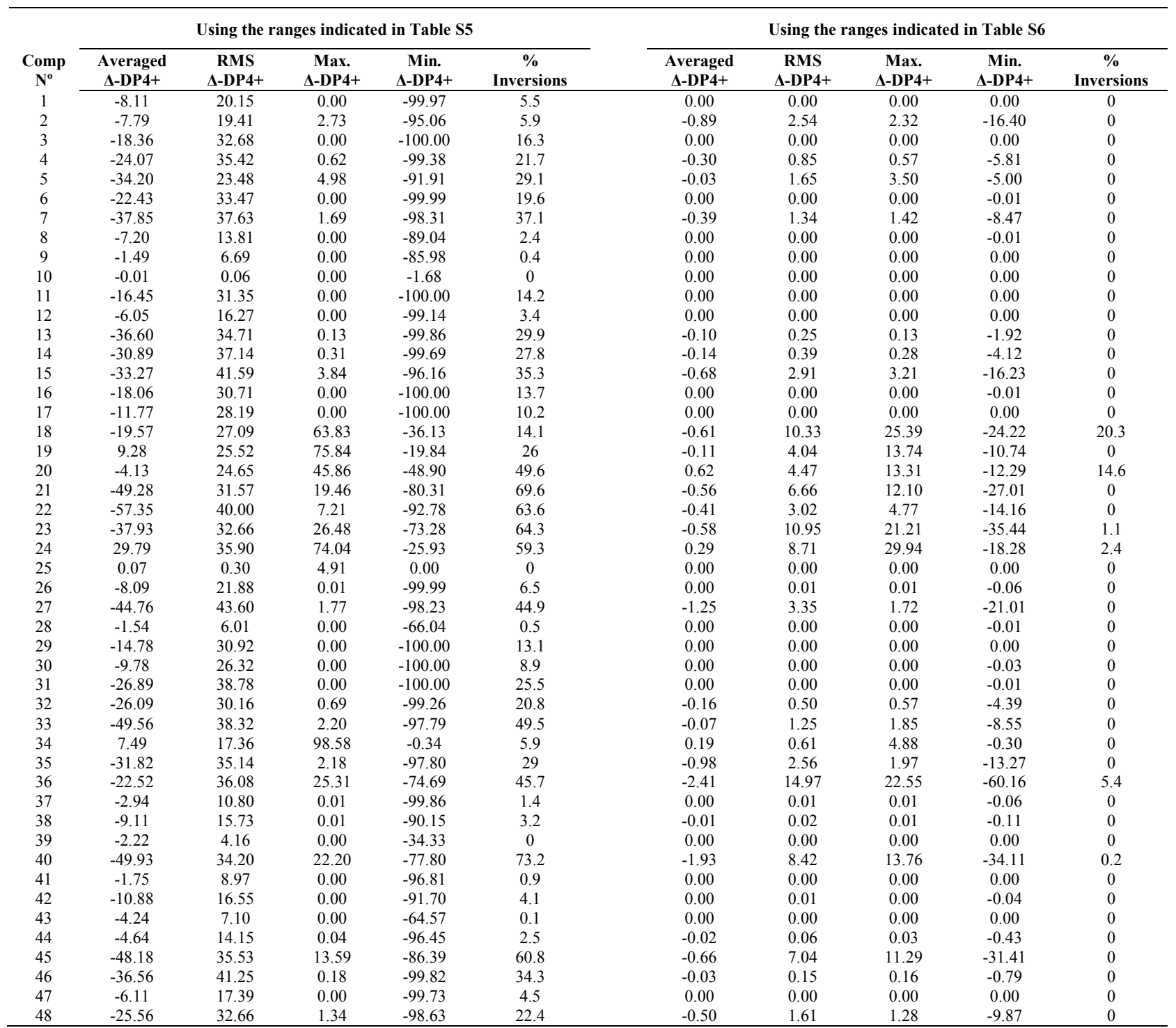


Table S12. $[\mu, \sigma]$ values estimated using the reduced set of 8 molecules (Figure 5), and the full set of 77 molecules (J. Org. Chem. 2015, 80, 12526) at 24 levels of theory.

\begin{tabular}{|c|c|c|c|c|c|c|c|c|c|c|}
\hline & \multicolumn{5}{|c|}{ Using the reduced set of 8 molecules } & \multicolumn{5}{|c|}{ Using the full set of 77 molecules } \\
\hline & ${ }^{13} \mathrm{C} \sigma_{\mathrm{s}}$ & ${ }^{13} \mathrm{C} \mu_{\mathrm{u}, \mathrm{sp} 2}$ & ${ }^{13} \mathrm{C} \sigma_{\mathrm{u}, \mathrm{sp} 2}$ & ${ }^{13} \mathrm{C} \mu_{\mathrm{u}, \mathrm{sp} 3}$ & ${ }^{13} \mathrm{C} \sigma_{\mathrm{u}, \mathrm{sp} 3}$ & ${ }^{13} \mathrm{C} \sigma_{\mathrm{s}}$ & ${ }^{13} \mathrm{C} \mu_{\mathrm{u}, \mathrm{sp} 2}$ & ${ }^{13} \mathrm{C} \sigma_{\mathrm{u}, \mathrm{sp} 2}$ & ${ }^{13} \mathrm{C} \mu_{\mathrm{u}, \mathrm{sp} 3}$ & ${ }^{13} \mathrm{C} \sigma_{\mathrm{u}, \mathrm{sp} 3}$ \\
\hline B3LYP/6-31G* (B1V) & 1.656 & -6.006 & 2.808 & 1.430 & 1.416 & 1.581 & -6.157 & 2.488 & 1.302 & 1.648 \\
\hline B3LYP/6-31G** (B2V) & 1.846 & -4.497 & 2.699 & 2.154 & 1.587 & 1.852 & -4.616 & 2.455 & 2.047 & 1.765 \\
\hline $\mathrm{B} 3 \mathrm{LYP} / 6-31+\mathrm{G} * *(\mathrm{~B} 3 \mathrm{~V})$ & 2.045 & -2.682 & 2.325 & 3.523 & 1.834 & 2.087 & -2.682 & 2.294 & 3.584 & 1.899 \\
\hline B3LYP/6-311G* (B4V) & 1.981 & 5.676 & 2.267 & 4.574 & 2.268 & 1.921 & 5.918 & 2.349 & 4.733 & 2.285 \\
\hline B3LYP/6-311G** (B5V) & 1.967 & 6.305 & 2.350 & 5.092 & 2.245 & 1.837 & 6.538 & 2.241 & 5.265 & 2.212 \\
\hline B3LYP/6-311+G** (B6V) & 1.891 & 7.267 & 2.088 & 5.030 & 2.354 & 1.833 & 7.371 & 2.252 & 5.157 & 2.294 \\
\hline $\mathrm{PCM} / \mathrm{B} 3 \mathrm{LYP} / 6-31 \mathrm{G}^{*}(\mathrm{~B} 1 \mathrm{P})$ & 1.655 & -4.960 & 2.267 & 1.713 & 1.403 & 1.525 & -5.226 & 1.838 & 1.596 & 1.594 \\
\hline PCM/B3LYP/6-31G** (B2P) & 1.844 & -3.461 & 2.191 & 2.432 & 1.622 & 1.784 & -3.696 & 1.837 & 2.336 & 1.752 \\
\hline $\mathrm{PCM} / \mathrm{B} 3 \mathrm{LYP} / 6-31+\mathrm{G} * *(\mathrm{~B} 3 \mathrm{P})$ & 2.161 & -1.329 & 2.191 & 3.955 & 1.862 & 2.034 & -1.417 & 1.994 & 4.044 & 1.895 \\
\hline $\mathrm{PCM} / \mathrm{B} 3 \mathrm{LYP} / 6-311 \mathrm{G}^{*}(\mathrm{~B} 4 \mathrm{P})$ & 2.056 & 7.023 & 2.475 & 4.999 & 2.354 & 1.841 & 7.279 & 2.647 & 5.165 & 2.355 \\
\hline PCM/B3LYP/6-311G** (B5 P) & 2.018 & 7.612 & 2.466 & 5.495 & 2.315 & 1.738 & 7.809 & 2.409 & 5.678 & 2.271 \\
\hline $\mathrm{PCM} / \mathrm{B} 3 \mathrm{LYP} / 6-311+\mathrm{G} * *(\mathrm{~B} 6 \mathrm{P})$ & 2.028 & 8.729 & 2.721 & 5.464 & 2.463 & 1.803 & 8.919 & 3.064 & 5.599 & 2.395 \\
\hline mPW1PW91/6-31G* (M1V) & 1.416 & -4.628 & 2.725 & 0.971 & 1.243 & 1.267 & -4.787 & 2.410 & 0.745 & 1.484 \\
\hline mPW1PW91/6-31G** (M2V) & 1.533 & -3.241 & 2.595 & 1.617 & 1.310 & 1.446 & -3.346 & 2.304 & 1.410 & 1.516 \\
\hline mPW1PW91/6-31+G** (M3V) & 1.642 & -2.189 & 2.297 & 2.489 & 1.495 & 1.598 & -2.185 & 2.105 & 2.431 & 1.605 \\
\hline mPW1PW91/6-311G* (M4V) & 1.716 & 5.883 & 2.255 & 4.259 & 1.756 & 1.603 & 6.084 & 2.040 & 4.284 & 1.740 \\
\hline mPW1PW91/6-311G** (M5V) & 1.739 & 6.566 & 2.416 & 4.809 & 1.740 & 1.547 & 6.781 & 2.048 & 4.851 & 1.689 \\
\hline mPW1PW91/6-311+G** (M6V) & 1.652 & 7.462 & 2.065 & 4.721 & 1.839 & 1.582 & 7.515 & 1.965 & 4.748 & 1.814 \\
\hline $\mathrm{PCM} / \mathrm{mPW} 1 \mathrm{PW} 91 / 6-31 \mathrm{G} *$ (M1P) & 1.390 & -3.511 & 2.144 & 1.299 & 1.230 & 1.227 & -3.787 & 1.724 & 1.087 & 1.433 \\
\hline $\mathrm{PCM} / \mathrm{mPW} 1 \mathrm{PW} 91 / 6-31 \mathrm{G}^{* *}(\mathrm{M} 2 \mathrm{P})$ & 1.512 & -2.136 & 2.036 & 1.938 & 1.354 & 1.391 & -2.365 & 1.675 & 1.742 & 1.508 \\
\hline $\mathrm{PCM} / \mathrm{mPW} 1 \mathrm{PW} 91 / 6-31+\mathrm{G}^{* *}$ (M3P) & 1.770 & -0.799 & 2.071 & 2.948 & 1.532 & 1.557 & -0.920 & 1.748 & 2.909 & 1.600 \\
\hline $\mathrm{PCM} / \mathrm{mPW} 1 \mathrm{PW} 91 / 6-311 \mathrm{G}^{*}$ (M4P) & 1.801 & 6.163 & 2.361 & 3.608 & 1.853 & 1.560 & 6.321 & 2.310 & 3.641 & 1.858 \\
\hline $\mathrm{PCM} / \mathrm{mPW} 1 \mathrm{PW} 91 / 6-311 \mathrm{G} * *(\mathrm{M} 5 \mathrm{P})$ & 1.797 & 6.774 & 2.429 & 4.104 & 1.820 & 1.491 & 6.885 & 2.144 & 4.154 & 1.787 \\
\hline $\mathrm{PCM} / \mathrm{mPW} 1 \mathrm{PW} 91 / 6-311+\mathrm{G} * *(\mathrm{M} 6 \mathrm{P})$ & 1.785 & 7.810 & 2.504 & 4.051 & 1.961 & 1.575 & 7.911 & 2.702 & 4.072 & 1.935 \\
\hline
\end{tabular}

\begin{tabular}{|c|c|c|c|c|c|c|c|c|c|c|}
\hline & \multicolumn{5}{|c|}{ Using the reduced set of 8 molecules } & \multicolumn{5}{|c|}{ Using the full set of 77 molecules } \\
\hline & ${ }^{1} \mathrm{H} \sigma_{\mathrm{s}}$ & ${ }^{1} \mathrm{H} \mu \mu_{\mathrm{u}, \mathrm{sp} 2}$ & ${ }^{1} \mathrm{H} \sigma_{\mathrm{u}, \mathrm{sp} 2}$ & ${ }^{1} \mathbf{H} \mu_{\mathrm{u}, \mathrm{sp} 3}$ & ${ }^{1} \mathbf{H} \sigma_{\mathrm{u}, \mathrm{sp} 3}$ & ${ }^{1} \mathrm{H} \sigma_{\mathrm{s}}$ & ${ }^{1} \mathbf{H} \mu_{\mathrm{u}, \mathrm{sp} 2}$ & ${ }^{1} \mathrm{H} \sigma_{\mathrm{u}, \mathrm{sp} 2}$ & ${ }^{1} \mathbf{H} \mu_{\mathrm{u}, \mathrm{sp} 3}$ & ${ }^{1} \mathbf{H} \sigma_{\mathrm{u}, \mathrm{sp} 3}$ \\
\hline B3LYP/6-31G* (B1V) & 0.127 & -0.163 & 0.183 & -0.075 & 0.151 & 0.140 & -0.172 & 0.194 & -0.056 & 0.169 \\
\hline B3LYP/6-31G** (B2V) & 0.121 & 0.020 & 0.180 & -0.086 & 0.139 & 0.128 & 0.016 & 0.177 & -0.072 & 0.149 \\
\hline $\mathrm{B} 3 \mathrm{LYP} / 6-31+\mathrm{G} * *(\mathrm{~B} 3 \mathrm{~V})$ & 0.122 & 0.140 & 0.170 & -0.068 & 0.141 & 0.127 & 0.139 & 0.163 & -0.060 & 0.134 \\
\hline B3LYP/6-311G* (B4V) & 0.094 & -0.046 & 0.155 & -0.049 & 0.113 & 0.116 & -0.035 & 0.169 & -0.029 & 0.149 \\
\hline B3LYP/6-311G** (B5V) & 0.096 & 0.106 & 0.141 & -0.082 & 0.117 & 0.113 & 0.112 & 0.149 & -0.072 & 0.130 \\
\hline B3LYP/6-311+G** (B6V) & 0.106 & 0.164 & 0.146 & -0.054 & 0.131 & 0.119 & 0.163 & 0.145 & -0.051 & 0.135 \\
\hline PCM/B3LYP/6-31G* (B1P) & 0.127 & -0.086 & 0.180 & -0.027 & 0.143 & 0.129 & -0.091 & 0.172 & -0.013 & 0.151 \\
\hline $\mathrm{PCM} / \mathrm{B} 3 \mathrm{LYP} / 6-31 \mathrm{G}^{* *}(\mathrm{~B} 2 \mathrm{P})$ & 0.114 & 0.099 & 0.167 & -0.037 & 0.129 & 0.115 & 0.097 & 0.149 & -0.027 & 0.130 \\
\hline $\mathrm{PCM} / \mathrm{B} 3 \mathrm{LYP} / 6-31+\mathrm{G}^{* *}(\mathrm{~B} 3 \mathrm{P})$ & 0.109 & 0.232 & 0.147 & -0.008 & 0.127 & 0.110 & 0.238 & 0.132 & -0.003 & 0.119 \\
\hline $\mathrm{PCM} / \mathrm{B} 3 \mathrm{LYP} / 6-311 \mathrm{G}^{*}(\mathrm{~B} 4 \mathrm{P})$ & 0.087 & 0.045 & 0.144 & 0.004 & 0.099 & 0.103 & 0.063 & 0.148 & 0.019 & 0.126 \\
\hline PCM/B3LYP/6-311G** (B5 P) & 0.083 & 0.193 & 0.125 & -0.027 & 0.102 & 0.095 & 0.206 & 0.124 & -0.022 & 0.105 \\
\hline $\mathrm{PCM} / \mathrm{B} 3 \mathrm{LYP} / 6-311+\mathrm{G}^{* *}(\mathrm{~B} 6 \mathrm{P})$ & 0.090 & 0.259 & 0.121 & 0.008 & 0.112 & 0.099 & 0.267 & 0.119 & 0.008 & 0.115 \\
\hline mPW1PW91/6-31G* (M1V) & 0.116 & -0.044 & 0.168 & -0.078 & 0.136 & 0.128 & -0.044 & 0.185 & -0.065 & 0.156 \\
\hline mPW1PW91/6-31G** (M2V) & 0.112 & 0.138 & 0.164 & -0.093 & 0.126 & 0.120 & 0.140 & 0.167 & -0.085 & 0.136 \\
\hline mPW1PW91/6-31+G** (M3V) & 0.116 & 0.234 & 0.157 & -0.080 & 0.137 & 0.122 & 0.239 & 0.149 & -0.075 & 0.127 \\
\hline mPW1PW91/6-311G* (M4V) & 0.091 & 0.279 & 0.149 & 0.220 & 0.114 & 0.113 & 0.305 & 0.168 & 0.239 & 0.148 \\
\hline mPW1PW91/6-311G** (M5V) & 0.092 & 0.455 & 0.134 & 0.183 & 0.114 & 0.110 & 0.478 & 0.142 & 0.193 & 0.123 \\
\hline mPW1PW91/6-311+G** (M6V) & 0.102 & 0.498 & 0.143 & 0.206 & 0.128 & 0.117 & 0.516 & 0.138 & 0.209 & 0.131 \\
\hline $\mathrm{PCM} / \mathrm{mPW} 1 \mathrm{PW} 91 / 6-31 \mathrm{G} *$ (M1P) & 0.112 & 0.044 & 0.165 & -0.028 & 0.125 & 0.117 & 0.047 & 0.162 & -0.021 & 0.136 \\
\hline $\mathrm{PCM} / \mathrm{mPW} 1 \mathrm{PW} 91 / 6-31 \mathrm{G} * *(\mathrm{M} 2 \mathrm{P})$ & 0.102 & 0.225 & 0.150 & -0.042 & 0.113 & 0.105 & 0.233 & 0.136 & -0.038 & 0.116 \\
\hline $\mathrm{PCM} / \mathrm{mPW} 1 \mathrm{PW} 91 / 6-31+\mathrm{G}^{* *}(\mathrm{M} 3 \mathrm{P})$ & 0.102 & 0.332 & 0.133 & -0.019 & 0.122 & 0.104 & 0.347 & 0.118 & -0.018 & 0.112 \\
\hline PCM/mPW1PW91/6-311G* (M4P) & 0.085 & 0.111 & 0.139 & -0.001 & 0.100 & 0.101 & 0.133 & 0.147 & 0.009 & 0.125 \\
\hline $\mathrm{PCM} / \mathrm{mPW} 1 \mathrm{PW} 91 / 6-311 \mathrm{G} * *$ (M5P) & 0.078 & 0.277 & 0.116 & -0.040 & 0.098 & 0.093 & 0.297 & 0.117 & -0.039 & 0.098 \\
\hline $\mathrm{PCM} / \mathrm{mPW} 1 \mathrm{PW} 91 / 6-311+\mathrm{G} * *$ (M6P) & 0.087 & 0.326 & 0.116 & -0.012 & 0.111 & 0.098 & 0.344 & 0.112 & -0.014 & 0.111 \\
\hline
\end{tabular}


Table S13. DP4+ values calculated for the test set (Figure 2) when correlating the GIAO NMR chemical shifts computed at the PCM/mPW1PW91/6$31+\mathrm{G}^{* *} / / \mathrm{B} 3 \mathrm{LYP} / 6-31 \mathrm{G}^{*}$ level of theory (M3P) with a) the $[\mu, \sigma, v]_{0}$ estimated from the full set of 77 molecules $(\mathrm{J}$. Org. Chem. 2015, 80, 12526) at the same level of theory; b) using the $[\mu, \sigma]$ terms estimated from the reduced set of 8 molecules (Figure 5) and the $[v]$ terms estimated from the full set of 77 molecules at the same level of theory; and c) using the $[\mu, \sigma]$ terms estimated from the reduced set of 8 molecules (Figure 5) at the same level of theory and the averaged $[v]$ terms of the 24 different levels of theory originally evaluated (Table S14).

\begin{tabular}{|c|c|c|c|}
\hline Comp. & $\begin{array}{c}\text { DP4+ } \\
\text { (a) }\end{array}$ & $\begin{array}{c}\text { DP4+ } \\
\text { (b) }\end{array}$ & $\begin{array}{c}\text { DP4+ } \\
\text { (c) }\end{array}$ \\
\hline 1 & 100.00 & 100.00 & 100.00 \\
\hline 2 & 97.27 & 98.28 & 99.33 \\
\hline 3 & 100.00 & 100.00 & 100.00 \\
\hline 4 & 99.38 & 99.61 & 99.88 \\
\hline 5 & 93.40 & 93.87 & 95.70 \\
\hline 6 & 100.00 & 99.99 & 100.00 \\
\hline 7 & 98.31 & 97.34 & 98.11 \\
\hline 8 & 100.00 & 99.99 & 100.00 \\
\hline 9 & 100.00 & 100.00 & 100.00 \\
\hline 10 & 100.00 & 100.00 & 100.00 \\
\hline 11 & 100.00 & 100.00 & 100.00 \\
\hline 12 & 100.00 & 100.00 & 100.00 \\
\hline 13 & 99.86 & 99.90 & 99.98 \\
\hline 14 & 99.69 & 99.73 & 99.87 \\
\hline 15 & 96.16 & 93.57 & 94.27 \\
\hline 16 & 100.00 & 100.00 & 100.00 \\
\hline 17 & 100.00 & 100.00 & 100.00 \\
\hline 18 & 36.13 & 29.21 & 25.36 \\
\hline 19 & 19.85 & 18.73 & 14.20 \\
\hline 20 & 49.27 & 52.24 & 53.12 \\
\hline 21 & 80.31 & 65.96 & 65.06 \\
\hline 22 & 92.78 & 86.81 & 87.59 \\
\hline 23 & 73.30 & 63.48 & 55.72 \\
\hline 24 & 25.95 & 34.35 & 39.61 \\
\hline 25 & 0.00 & 0.00 & 0.00 \\
\hline 26 & 99.99 & 99.99 & 100.00 \\
\hline 27 & 98.23 & 98.26 & 99.19 \\
\hline 28 & 100.00 & 100.00 & 100.00 \\
\hline 29 & 100.00 & 100.00 & 100.00 \\
\hline 30 & 100.00 & 100.00 & 100.00 \\
\hline 31 & 100.00 & 100.00 & 100.00 \\
\hline 32 & 99.30 & 99.47 & 99.81 \\
\hline 33 & 97.79 & 95.64 & 93.86 \\
\hline 34 & 0.34 & 0.40 & 0.04 \\
\hline 35 & 97.81 & 98.42 & 99.40 \\
\hline 36 & 74.69 & 74.95 & 79.90 \\
\hline 37 & 99.98 & 99.98 & 100.00 \\
\hline 38 & 99.99 & 99.99 & 100.00 \\
\hline 39 & 100.00 & 100.00 & 100.00 \\
\hline 40 & 77.80 & 65.43 & 70.25 \\
\hline 41 & 100.00 & 100.00 & 100.00 \\
\hline 42 & 100.00 & 99.99 & 100.00 \\
\hline 43 & 100.00 & 100.00 & 100.00 \\
\hline 44 & 99.96 & 99.98 & 100.00 \\
\hline 45 & 86.39 & 77.60 & 73.97 \\
\hline 46 & 99.82 & 99.62 & 99.64 \\
\hline 47 & 100.00 & 100.00 & 100.00 \\
\hline 48 & 98.66 & 98.22 & 98.64 \\
\hline
\end{tabular}

Table S14. Averaged degrees of freedom for the 24 levels of theory evaluated in the original method (J. Org. Chem. 2015, 80, 12526)

\begin{tabular}{cc}
\hline Comp. & $\begin{array}{c}\text { Averaged } \\
\text { value }\end{array}$ \\
\hline$v_{13 \mathrm{Cu}, \mathrm{sp} 2}$ & 8 \\
$\boldsymbol{V}_{13 \mathrm{Cu}, \mathrm{sp} 3}$ & 10 \\
$\vee_{1 \mathrm{Hu}, \mathrm{sp} 2}$ & 8 \\
$\vee_{1 \mathrm{Hu}, \mathrm{sp} 3}$ & 4 \\
$\vee_{13 \mathrm{Cs}}$ & 7 \\
$v_{1 \mathrm{Hs}}$ & 4 \\
\hline
\end{tabular}


49

B3LYP/6-31G* Geometry

O $-0.656999-0.290213 \quad 1.291750$

C $1.365735-0.049958 \quad 0.003037$

C $-0.366875 \quad 1.660258-0.100968$

C $0.924546 \quad 1.337297-0.280410$

C -1.3289760 .6030640 .387409$

$\begin{array}{llll}\text { C } & 0.208287 & -0.979228 & 0.418648\end{array}$

O $2.511488-0.442992-0.118935$

C $-1.683919-0.390690-0.747345$

O $-0.556790-1.270253-0.758630$

H $-0.7509352 .651694-0.331034$

H $1.6746242 .045868-0.620307$

H $-2.197927 \quad 1.0186660 .900198$

$\begin{array}{llll}\mathrm{H} & 0.565290 & -1.900005 & 0.883929\end{array}$

H $-1.7916480 .076909-1.730108$

H - $2.593775-0.949930-0.498392$

SCF Energy (B3LYP/6-31G*)= -457.727973424

\section{0}

B3LYP/6-31G* Geometry

C $0.094694-1.9385140 .139020$

C $0.3278940 .905856-0.135142$

C $-1.044453-1.1354660 .030516$

C $1.321572-1.3004720 .096118$

C $1.4828640 .091024-0.044565$

C $-0.927402 \quad 0.279688-0.096795$

$\begin{array}{llll}\text { C } & -2.219858 & 1.096088 & -0.096734\end{array}$

C $-3.3666270 .350097-0.811464$

C $-3.466725-1.088260-0.375886$

C $-2.372390-1.7573750 .015149$

C $2.9255920 .306058-0.050116$

$\begin{array}{lllll}\text { C } & 3.471303 & -0.930311 & 0.083344\end{array}$

$\begin{array}{llll}\text { O } & 2.535111 & -1.927711 & 0.175154\end{array}$

C $3.721046 \quad 1.573270-0.170767$

$\begin{array}{llll}\text { C } & 0.438181 & 2.410515 & -0.274229\end{array}$

C $-2.620110 \quad 1.465289 \quad 1.349194$

H $\quad 0.022981-3.017483 \quad 0.236107$

H $-2.052815 \quad 2.030723-0.642854$

H $-3.2079230 .382953-1.902108$

H $-4.3116410 .877807-0.629268$

H $-4.431786-1.587737-0.424690$

H $-2.432014-2.8092220 .288548$

H $\quad 4.499960-1.2586690 .128439$

H $3.512390 \quad 2.103075-1.108026$

H 3.5150962 .2690890 .651668

H $4.793687 \quad 1.352430-0.150946$

H $0.0329302 .756653-1.233973$

H $-0.1179602 .933543 \quad 0.512155$

H $1.4725042 .748871-0.218736$

H $-1.8160592 .006644 \quad 1.859233$

H $-3.5138662 .101891 \quad 1.352981$

H $-2.839866 \quad 0.566208 \quad 1.934388$

SCF Energy (B3LYP/6-31G*)= -656.187084929

51

B3LYP/6-31G* Geometry

C $2.988308-1.844583-0.315754$

$\begin{array}{llll}\text { C } & 3.671012 & 0.881356 & -0.004758\end{array}$

C $4.306723-1.417249-0.511710$

C $2.012752-0.9129270 .023227$

$\begin{array}{llll}\text { C } & 2.358994 & 0.438614 & 0.172234\end{array}$

C $4.636142-0.069068-0.353170$

$\begin{array}{lllll}\mathrm{N} & 1.208782 & 1.197824 & 0.488546\end{array}$

C 0.0010390 .3359830 .415160

C $0.553157-1.1282790 .365203$

C $-0.8136210 .836220-0.792920$

C -1.4198312 .203598 - 0.412241

$\begin{array}{lllll}\text { C } & -0.358863 & 3.075492 & 0.317285\end{array}$

$\begin{array}{llll}\text { C } & 1.081832 & 2.562750 & 0.313884\end{array}$

$\begin{array}{lllll}\text { O } & 2.038506 & 3.317170 & 0.193685\end{array}$

C $-0.269085-2.078802-0.557814$

C $-0.819824-1.405154-1.817400$

C $-1.739351-0.243038-1.393277$ 
C $-2.820926-0.767441-0.447882$

$\begin{array}{lllll}\text { O } & -2.523142 & 2.141724 & 0.487902\end{array}$

$\begin{array}{llll}\text { C } & -3.716892 & 0.034901 & 0.138757\end{array}$

C $-3.713913 \quad 1.536322-0.022134$

C $\quad 0.365521-1.839019 \quad 1.730409$

C $-1.099638-2.280999 \quad 1.702749$

$\mathrm{N}-1.368526-2.618630 \quad 0.292702$

C $-2.721346-2.250637-0.154978$

H $2.733344-2.895895-0.425863$

H $3.914613 \quad 1.9280320 .113936$

H $5.074470-2.137117-0.781001$

H $5.6628230 .254948-0.501891$

$\begin{array}{lllll}H & -0.600916 & 0.478841 & 1.318514\end{array}$

H $-0.085843 \quad 1.061316-1.586922$

H $-1.7488012 .706933-1.336013$

H -0.6863973 .1486061 .361972$

H $-0.3267024 .092721-0.077422$

H $0.389044-2.908651-0.853463$

H $\quad 0.010450-1.034393-2.429419$

H $-1.357559-2.137199-2.429794$

H -2.223096 $0.186797-2.283637$

H $-4.459754-0.3930520 .810922$

H $-4.533524 \quad 1.9814240 .549567$

H -3.853349 $1.827823-1.076992$

H $0.605322-1.1918202 .580162$

H $\quad 1.032217-2.7079231 .774725$

H - $1.751260-1.4622402 .050284$

H $-1.286587-3.1442442 .354075$

H $-3.432761-2.5456440 .622563$

H $-2.973578-2.843027-1.044949$

SCF Energy (B3LYP/6-31G*) $=-1073.02324315$

\section{2}

B3LYP/6-31G* Geometry

C $2.954171-1.3795610 .231135$

$\begin{array}{llll}\text { C } & 2.598969 & 1.110849 & 0.147894\end{array}$

C $4.3689310 .026251-1.213078$

C $3.607514 \quad 1.196739-0.739324$

C $3.966364-1.275803-0.641261$

$\begin{array}{llll}\text { C } & 2.157270 & -0.209273 & 0.750064\end{array}$

$\begin{array}{llll}\text { C } & 0.615543 & -0.437998 & 0.541027\end{array}$

$\begin{array}{lllll}\text { C } & -0.311139 & 0.798629 & 0.697668\end{array}$

$\begin{array}{lllll}\text { C } & 0.335774 & 2.119400 & 0.209949\end{array}$

C 1.8102262 .3105140 .591867

C $-1.6131820 .603295-0.107236$

C $-2.269945-0.791726-0.053090$

C $-1.309690-1.797516-0.702682$

C $0.103820-1.649381-0.165811$

C $-3.564527-0.535833-0.838030$

$\begin{array}{lllll}\text { C } & -3.907309 & 0.968190 & -0.770198\end{array}$

$\begin{array}{llll}\text { C } & -2.783438 & 1.587149 & 0.094457\end{array}$

O $-4.227647-1.376021-1.407904$

$\begin{array}{llll}\text { C } & -5.349500 & 1.232625 & -0.328438\end{array}$

$\begin{array}{llll}\text { O } & 0.207755 & -1.613981 & 1.267726\end{array}$

$\begin{array}{lllll}\text { O } & 5.283521 & 0.126723 & -2.027358\end{array}$

C $2.404240-0.1674162 .294593$

$\begin{array}{llll}\text { C } & -2.751182 & -1.247728 & 1.350827\end{array}$

H $2.690138-2.346842 \quad 0.653968$

H $3.9143842 .156111-1.149870$

H $4.537787-2.141484-0.965509$

H $-0.5371840 .891292 \quad 1.769626$

$\begin{array}{llll}\mathrm{H} & -0.257318 & 2.961968 & 0.583507\end{array}$

H $0.2718852 .154826-0.885934$

$\begin{array}{lllll}\mathrm{H} & 1.902694 & 2.452708 & 1.676778\end{array}$

H $\quad 2.201415 \quad 3.2182050 .119984$

H -1.307898 $0.717717-1.162000$

H - $1.640487-2.830407-0.537928$

H -1.289171 -1.649144 -1.790821

H $\quad 0.837665-2.298109-0.645457$

H $-3.7931791 .331048-1.803613$

H $-3.083256 \quad 1.629693 \quad 1.149630$

H $-2.5411322 .608907-0.214309$

H $-5.507995 \quad 0.921870 \quad 0.710974$

$\begin{array}{llll}H & -6.045379 & 0.670849 & -0.959214\end{array}$

H $-5.5953212 .297652-0.402772$

$\begin{array}{lllll}\mathrm{H} & 1.796362 & 0.599371 & 2.783361\end{array}$

H $2.141785-1.1325382 .733200$ 
H $3.458511 \quad 0.0438212 .496994$

H $-1.909607-1.3909032 .028356$

H $-3.446659-0.529473 \quad 1.799253$

H $-3.284536-2.1993391 .250080$

SCF Energy (B3LYP/6-31G*)= -1001.82528387

53

B3LYP/6-31G* Geometry

C $1.016229-0.6659261 .294706$

$\begin{array}{llll}\text { C } & 1.053494 & 0.673575 & 1.280253\end{array}$

C $1.096202 \quad 1.117515-0.173359$

C $-0.3142220 .802796-0.815383$

C $1.025729-1.142050-0.151737$

C $-0.360439-0.770580-0.794542$

C $1.916775-0.044633-0.787108$

C $-1.544430 \quad 1.213768-0.052026$

C $-1.645481-1.160179-0.014376$

$\begin{array}{llll}\text { C } & -2.249977 & 0.166866 & 0.384517\end{array}$

H $\quad 0.893560-1.307712 \quad 2.161386$

$\mathrm{H} \quad 0.959064 \quad 1.3406812 .131118$

H $1.4528752 .134134-0.358488$

H $-0.319954 \quad 1.205301-1.837891$

H $1.325623-2.181255-0.312850$

H $-0.391170-1.172607-1.812555$

H $2.950673-0.073345-0.428404$

H $1.907013-0.054195-1.884498$

H $-1.807596 \quad 2.2547910 .120860$

H $-1.428760-1.7835520 .864902$

H $-2.338634-1.743824-0.636517$

H -3.1659720 .2346640 .967270$

SCF Energy (B3LYP/6-31G*) $=-388.069499378$

\section{4}

B3LYP/6-31G* Geometry

C $-3.4560310 .680002-0.000029$

C $-1.796075-1.491099-0.000448$

$\begin{array}{llll}\text { C } & -2.065140 & 0.941185 & -0.000192\end{array}$

$\begin{array}{llll}\text { C } & -3.927878 & -0.620087 & 0.000128\end{array}$

$\mathrm{N}-3.095458-1.704725-0.000215$

C $-1.205928-0.202924-0.000256$

$\begin{array}{lllll}C & 0.183944 & 0.052948 & -0.000065\end{array}$

$\begin{array}{lllll}\text { C } & 0.702659 & 1.334176 & 0.000004\end{array}$

$\begin{array}{llll}\text { C } & -0.154680 & 2.459883 & -0.000054\end{array}$

C $-1.514952 \quad 2.261924-0.000142$

$\begin{array}{llll}\text { C } & 2.164360 & 1.207331 & 0.000128\end{array}$

$\begin{array}{llll}\text { C } & 2.390098 & -0.277271 & 0.000058\end{array}$

O $1.136518-0.920147-0.000005$

C $3.521167-1.0069850 .000066$

C $4.874430-0.3516640 .000148$

C $3.492497-2.5106610 .000026$

$\begin{array}{lllll}\text { O } & 3.007725 & 2.097795 & 0.000085\end{array}$

C $-5.399885-0.937732 \quad 0.000529$

H $-4.154620 \quad 1.512524-0.000020$

H - $1.153016-2.369946-0.000852$

H $\quad 0.276093 \quad 3.456766-0.000003$

H $-2.1969613 .107506-0.000129$

H $5.446833-0.675926-0.879852$

$\mathrm{H} \quad 4.8036650 .735775 \quad 0.000220$

H $5.446778-0.676053 \quad 0.880137$

H $4.023819-2.897951-0.879698$

H $4.023381-2.897987 \quad 0.880000$

H $2.477508-2.910174-0.000223$

H $-5.659635-1.537269-0.879612$

$\mathrm{H}-5.658880-1.538245 \quad 0.880260$

H $-6.012209-0.0312890 .001234$

SCF Energy $\left(B 3 L Y P / 6-31 G^{*}\right)=-784.350313185$

\section{5}

B3LYP/6-31G* Geometry

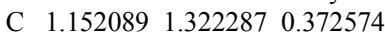

$\begin{array}{llll}\text { C } & -1.353640 & 0.781927 & 0.655679\end{array}$

C $-0.636606 \quad 3.049808-0.200688$

C $-1.696938 \quad 1.945722-0.289411$

$\begin{array}{llll}\text { C } & 0.785709 & 2.547553 & -0.506382\end{array}$

$\begin{array}{lllll}\text { C } & 0.052045 & 0.222948 & 0.365196\end{array}$

C $0.058426-0.561024-0.959131$

O $-1.071579-1.429329-1.157144$ 
C $-2.290922-1.264105-0.566019$

$\begin{array}{llll}\text { C } & -2.366887 & -0.382267 & 0.674215\end{array}$

$\begin{array}{lllll}C & 2.546436 & 0.751884 & 0.016547\end{array}$

$\begin{array}{llll}\text { C } & 2.864085 & -0.665279 & 0.527802\end{array}$

C $1.844512-1.740197 \quad 0.084020$

O $1.212840-1.324223-1.142759$

O $-3.228265-1.884992-1.005139$

$\begin{array}{llll}\text { C } & -3.812199 & 0.040090 & 0.956984\end{array}$

C $1.7998843 .691887-0.354278$

$\begin{array}{lllll}\text { O } & 0.350453 & -0.644365 & 1.495044\end{array}$

$\begin{array}{lllll}\text { O } & 0.840263 & -1.948116 & 1.057268\end{array}$

C $2.446402-3.113416-0.164080$

$\begin{array}{lllll}\text { H } & 1.177674 & 1.662370 & 1.418486\end{array}$

H - $1.314767 \quad 1.1912361 .675659$

H $-0.653085 \quad 3.480903 \quad 0.811808$

H $-0.8884973 .867535-0.887798$

H -2.677889 2.353284 -0.022561

H $-1.787268 \quad 1.590419-1.326365$

H $\quad 0.807225 \quad 2.226512-1.559894$

H $\quad 0.0319800 .147770-1.791636$

H -2.038345 - 1.0449121 .487011

H $3.314918 \quad 1.429408 \quad 0.404462$

H $2.660598 \quad 0.749046-1.074232$

H $2.930781-0.6945641 .620050$

H $3.849113-0.9472640 .137656$

H $-4.445007-0.841547 \quad 1.078580$

$\begin{array}{lllll}\text { H } & -4.232449 & 0.630939 & 0.137273\end{array}$

$\begin{array}{llll}\text { H } & -3.861537 & 0.636486 & 1.875288\end{array}$

H 1.8727464 .0135550 .692731

H $2.8016793 .406335-0.689087$

H $1.4895734 .560107-0.947034$

H $3.038556-3.4320190 .698542$

H $\quad 1.649780-3.838534-0.348510$

H $3.090155-3.065770-1.046763$

SCF Energy $\left(B 3 L Y P / 6-31 G^{*}\right)=-960.542460074$

\section{6}

B3LYP/6-31G* Geometry

C $1.630118-1.753407-0.499911$

C $2.3635270 .644888-0.110922$

C $4.104541-1.227468-0.356626$

$\begin{array}{lllll}\text { C } & 3.856957 & 0.212691 & 0.154007\end{array}$

C $3.023333-2.264517-0.099468$

C $1.248626-0.4234800 .197691$

C $-0.1110760 .138422-0.389819$

$\begin{array}{llll}\text { C } & -0.467013 & 1.494881 & 0.271009\end{array}$

$\begin{array}{llll}\text { C } & 0.640530 & 2.526510 & 0.023107\end{array}$

C 2.0126342 .0220150 .494066

C $-1.862046 \quad 1.978377-0.147017$

C $-2.8506860 .847055-0.079395$

C $-2.408820-0.433926-0.000886$

O $-1.136691-0.851708-0.011901$

O $-3.220323-1.5129330 .112858$

C $-4.581124-1.321815 \quad 0.146832$

$\begin{array}{llll}\text { C } & -5.120601 & -0.090527 & 0.062188\end{array}$

C $-4.287585 \quad 1.113622-0.056709$

$\begin{array}{llllll}\text { O } & -4.756137 & 2.253431 & -0.122328\end{array}$

$\begin{array}{llll}\text { C } & -5.304172 & -2.622921 & 0.280938\end{array}$

O $5.138969-1.532795-0.921768$

$\begin{array}{llll}\text { C } & 4.279343 & 0.233711 & 1.647761\end{array}$

C $4.809443 \quad 1.155739-0.611282$

C $1.057350-0.703231 \quad 1.711780$

C $-0.1708320 .204448-1.926736$

H $\quad 0.874821-2.517594-0.284850$

H $1.634964-1.615659-1.586600$

$\begin{array}{llll}\text { H } & 2.333585 & 0.777742 & -1.201477\end{array}$

H $3.048934-2.537508 \quad 0.963815$

H $3.289528-3.164339-0.661889$

$\begin{array}{lllll}\mathrm{H} & -0.514768 & 1.307966 & 1.350648\end{array}$

$\begin{array}{lllll}\mathrm{H} & 0.386165 & 3.458078 & 0.544257\end{array}$

H $0.6822292 .777565-1.045614$

$\begin{array}{lllll}\mathrm{H} & 2.021399 & 1.970590 & 1.589473\end{array}$

$\begin{array}{lllll}\text { H } & 2.776249 & 2.756437 & 0.218473\end{array}$

H -2.1996282 .7816660 .518282$

H $-1.8492552 .422536-1.152624$

H -6.1972520 .0343920 .088800$

H $-5.007319-3.1382381 .202129$ 
H $-6.383482-2.457263 \quad 0.302253$

H $-5.064748-3.287853-0.557350$

H $\quad 5.338870-0.032310 \quad 1.729529$

H $3.712734-0.4595432 .272860$

H $4.157224 \quad 1.2376892 .066864$

H $5.832580 \quad 0.778693 \quad-0.553789$

H $4.543901 \quad 1.216137-1.672795$

H $4.7906232 .166780-0.193643$

H $\quad 1.856661-1.3314342 .107113$

H $\quad 0.118421-1.237305 \quad 1.875092$

$\begin{array}{lllll}\mathrm{H} & 1.039014 & 0.203294 & 2.320674\end{array}$

$\begin{array}{lllll}\mathrm{H} & 0.619401 & 0.826875 & -2.351890\end{array}$

H $-0.093998-0.794660-2.359350$

H - $1.1280660 .622902-2.246899$

SCF Energy (B3LYP/6-31G*) $=-1117.56934573$ 